\title{
Beta Function and Schwinger Functions for a Many Fermions System in One Dimension. Anomaly of the Fermi Surface
}

\author{
G. Benfatto ${ }^{1}$, G. Gallavotti ${ }^{2,3}$, A. Procacci $^{2}$, B. Scoppola ${ }^{4}$ \\ 1 Dipartimento di Matematica, Università di Roma “Tor Vergata," I-00133 Roma, Italy \\ 2 Dipartimento di Fisica, Università di Roma "La Sapienza," P. Moro 5, I-00185 Roma, Italy \\ 3 Mathematics Dept., Hill Center, Rutgers University, New Brunswick, NJ 08903, USA \\ 4 Dipartimento di Matematica, Università di Roma "La Sapienza," I-00185 Roma, Italy
}

Received: 25 February 1993/in revised form: 28 April 1993

\begin{abstract}
We present a rigorous discussion of the analyticity properties of the beta function and of the effective potential for the theory of the ground state of a one dimensional system of many spinless fermions. We show that their analyticity domain as a function of the running couplings is a polydisk with positive radius bounded below, uniformly in all the cut offs (infrared and ultraviolet) necessary to give a meaning to the formal Schwinger functions. We also prove the vanishing of the scale independent part of the beta function showing that this implies the analyticity of the effective potential and of the Schwinger functions in terms of the bare coupling. Finally we show that the pair Schwinger function has an anomalous long distance behaviour.
\end{abstract}

\section{Contents}

1 Introduction $\quad . \quad . \quad . \quad . \quad$. . . . . . . . . . . . . . . . . . . . . . 94

2 Functional Integral Representation of Fermionic Correlation Functions . . . . . . . 98

3 Ultraviolet Limit for the Effective Potential … . . . . . . . . . . . . . . . 106

4 The Effective Potential in the Infrared Region. Failure of Normal Scaling . . . . . 121

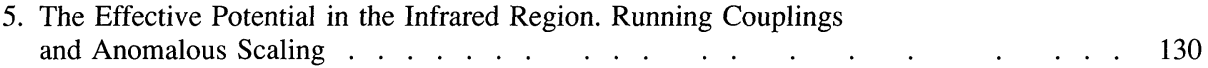

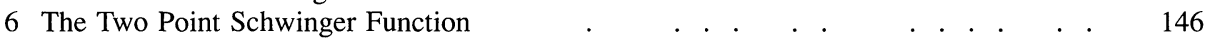

7. The Vanishing of the Beta Function and Completion of the Theory of Spinless Fermi Systems ． . . . . . . . . . . . . . . . . . . . . . . 151

Appendix 1. Bounds on the Free Propagators . . . . . . . . . . . . . . . . . 157

Appendix 2. The Gramm-Hadamard (and Related) Inequalities . . . . . . . . . . . 159

Appendix 3 The Bound (5.60) . . . . . . . . . . . . . . . . 164

Appendix 4. Simplified Beta Functional . . . . . . . . . . . . . . . . 169

References . . . . . . . . . . . . . . . . . . . . . 170 


\section{Introduction}

In this paper we study a system of interacting one dimensional fermions. The Hamiltonian for $n$ spinless particles in a periodic box of length $L$ will be:

$$
H=\sum_{i=1}^{n}\left(\frac{-\Delta_{\vec{x}_{i}}}{2 m}-\mu\right)+2 \lambda \sum_{i<j} \bar{v}\left(\vec{x}_{i}-\vec{x}_{j}\right),
$$

where $m>0$ is the particles' mass, $\mu$ is the chemical potential, $2 \lambda \bar{v}(\vec{r})$ is the pair potential, which we suppose bounded, smooth, even in $\vec{r}$ and with finite range $p_{0}^{-1}$.

Physically one defines the Fermi momentum $p_{F}$ so that the ground state energy of $H$ has the minimum at $n=2 p_{F} L / 2 \pi$ when $\mu=p_{F}^{2} / 2 m$, while the mass of particles is defined by computing the minimum energy increase obtained by adding one particle to the ground state.

Usually one requires that $p_{F}$ has a given value and that the minimum energy increase has the form:

$$
e\left(\vec{k}_{0}\right)=\left(\vec{k}_{0}^{2}-p_{F}^{2}\right) / 2 m,
$$

where $\vec{k}_{0}$ is the smallest $\vec{k}$ of the form $2 \pi s L^{-1}, s$ integer, larger than $p_{F}$; this, however, cannot be imposed on (1.1) as there are not free parameters.

Hence we shall study:

$$
\begin{aligned}
H & =\sum_{i=1}^{n}\left(\frac{-\Delta_{\vec{x}_{i}}}{2 m}-\mu\right)+\alpha \sum_{i=1}^{n}\left(\frac{-\Delta_{\vec{x}_{i}}}{2 m}-\mu\right)+\nu n+2 \lambda \sum_{i<j} \bar{v}\left(\vec{x}_{i}-\vec{x}_{j}\right) \\
& \equiv T+\alpha T+\nu N+\bar{V}
\end{aligned}
$$

and tune $\alpha, \nu$ so that the ground state of (1.3) has the above two properties. We require, given $p_{F}, m>0$, that $p_{F}$ and $L$ are so related that $2 \pi L^{-1}\left(n_{F}+1 / 2\right)=p_{F}$ with $n_{F}$ integer; this implies, in particular, that no particle can have momentum $\pm p_{F}$ and that $\vec{k}_{0}=p_{F}+\frac{\pi}{L}$.

It is very useful to write the Hamiltonian $H$ in second quantization, i.e. in terms of creation and annihilation operators $a_{k}^{+}, a_{k}^{-}$. Defining:

$$
\psi_{\vec{x}}^{ \pm}=L^{-1 / 2} \sum_{\vec{k}} e^{ \pm i \vec{k}} \vec{x} a_{\vec{k}}^{ \pm}
$$

we have:

$$
\begin{aligned}
& T=\sum_{\vec{k}}\left(\frac{\vec{k}^{2}}{2 m}-\mu\right) a_{\vec{k}}^{+} a_{\vec{k}}^{-}=\int_{L} d \vec{x}\left(\frac{1}{2 m} \vec{\partial} \psi_{\vec{x}}^{+} \vec{\partial} \psi_{\vec{x}}^{-}-\mu \psi_{\vec{x}}^{+} \psi_{\vec{x}}^{-}\right), \\
& N=\sum_{\vec{k}} a_{\vec{k}}^{+} a_{\vec{k}}^{-}=\int_{L} d \vec{x} \psi_{\vec{x}}^{+} \psi_{\vec{x}}^{-}, \\
& \bar{V}=\lambda \int_{L \times L} d \vec{x} d \vec{y} \bar{v}(\vec{x}-\vec{y}) \psi_{\vec{x}}^{+} \psi_{\vec{y}}^{+} \psi_{\vec{y}}^{-} \psi_{\vec{x}}^{-} .
\end{aligned}
$$

Let us denote $E^{(n)}$ the ground state energy of the system with $n$ particles and let us define $N=2 n_{F}+1$. 
By first order perturbation theory it is easy to see that:

$$
\begin{aligned}
E^{(N+1)}-E^{(N)}= & (1+\alpha) e\left(p_{F}+\frac{\pi}{L}\right) \\
& +\nu+2 \lambda \frac{2 \pi}{L} \sum_{\vec{k}: e(\vec{k})<0}\left[\hat{v}(0)-\hat{v}\left(p_{F}+\frac{\pi}{L}-\vec{k}\right)\right], \\
E^{(N-1)}-E^{(N)}= & -(1+\alpha) e\left(p_{F}-\frac{\pi}{L}\right) \\
& -\nu-2 \lambda \frac{2 \pi}{L} \sum_{\vec{k}: e(\vec{k})<0}\left[\hat{v}(0)-\hat{v}\left(p_{F}-\frac{\pi}{L}-\vec{k}\right)\right],
\end{aligned}
$$

where $2 \pi \hat{v}(\vec{k})=\int d \vec{x} e^{-i \vec{k} \vec{x}} \bar{v}(\vec{x})$.

The conditions that the system has $p_{F}$ as Fermi momentum and $m$ as mass in the presence of interaction can be translated in the conditions:

$$
E^{(N \pm 1)}-E^{(N)}= \pm e\left(p_{F} \pm \frac{\pi}{L}\right)
$$

which imply, by using $\hat{v}\left(p_{F} \pm \frac{\pi}{L}-\vec{k}\right)=\hat{v}\left(p_{F}-\vec{k}\right) \pm \frac{\pi}{L} \hat{v}^{\prime}\left(p_{F}-\vec{k}\right)+O\left(L^{-2}\right)$, that:

$$
\begin{gathered}
\nu+2 \lambda \int_{e(\vec{k})<0} d \vec{k}\left[\hat{v}(0)-\hat{v}\left(p_{F}-\vec{k}\right)\right]+O\left(L^{-1}\right)=0, \\
\alpha \frac{p_{F}}{m}-2 \lambda \int_{e(\vec{k})<0} d \vec{k} \hat{v}^{\prime}\left(p_{F}-\vec{k}\right)+O\left(L^{-1}\right)=0 .
\end{gathered}
$$

Recursively one can determine the higher order corrections to $\alpha, \nu$, pursuing with formal perturbation theory. If one, however, attempts at estimating the remainders one meets serious difficulties, unless one is willing to take $\lambda$ so small to be of order much smaller than $L^{-1}$, say of $O\left(\eta L^{-1}\right)$ for some small $\eta$. In the latter case one can easily check that there are no convergence problems for the perturbation expansions (and in fact the first order is dominant), as it is physically obvious. For $\eta$ small enough and $L$ fixed the perturbation theory converges, the ground state is unique and separated by a gap of order $L^{-1} p_{F} / m$ from the first excited state.

One possible approach to the theory of low temperature Fermi gases that we shall follow, is to study the above perturbation expansions as well as the expansions of the other interesting quantities (like the system reduced density matrices or the Schwinger functions, see Sect. 2), and to show that they can be resummed so that, after resummation, they admit analytic continuation in $\lambda$ up to $\lambda$ 's of size of order 1 uniformly in $L$ and $\beta$.

If this goal is achieved, it is clear that we have constructed objects of interest for low temperature physics: they can be interpreted as Gibbs states of the system provided they verify the necessary positivity properties. The latter are, essentially, automatically verified as we know that for $L, \beta>0$ fixed none of the correlation functions has a singularity for $\lambda, \alpha, \nu$ real (small or large).

In this paper we study a resummation algorithm, generated by the application of the renormalization group methods to the study of the above series. We show that 
the resummation can be described in terms of stability properties of a well defined dynamical system.

We call beta function the functions defining the dynamical system iteration map $B_{h}$ : the latter operates on a three dimensional set of parameters called the running couplings denoted by $\underline{r}$. Each triple $\underline{r}_{0}$ of initial data generates, for $h=0,-1,-2, \ldots$ a trajectory $\underline{r}_{h-1}=\underline{r}_{h}+B_{h}\left(\underline{r}_{h}, \underline{r}_{h+1}, \ldots, \underline{r}_{0}\right)$ which, under the condition that $\left|\underline{r}_{h}\right|$ remains small, provides a set of parameters in terms of which the relevant dynamical quantities (Schwinger functions) can be expanded in a convergent power series

The reason we call the above a resummation is because the expansion constructed is not in a power series of $\underline{r}_{0}$ : if we express $\underline{r}_{h}$ in powers of $\underline{r}_{0}$ it may well be that the convergence radius of the expansion shrinks to zero as $h \rightarrow-\infty$.

Our main results are:

1) The existence and boundedness and analyticity of the functions $B_{h}\left(\underline{r}_{h}, \ldots, \underline{r}_{0}\right)$ as functions of their arguments (regarded as independent arguments), if they are small enough $\cdot\left|\underline{r}_{h}\right| \leq \varepsilon$, for all $h \leq 0$.

2) We also show that $B_{h}(\underline{r}, \underline{r}, \ldots, \underline{r}) \equiv \beta_{h}(\underline{r})$ is the sum of two parts $\beta_{h}(\underline{r})=$ $\beta(\underline{r})+\hat{\beta}_{h}(\underline{r})$ with $\hat{\beta}_{h}(\underline{r}) \rightarrow 0$, for $h \rightarrow-\infty$ and for $|\underline{r}| \leq \varepsilon$, exponentially fast, and with $\beta(\underline{r})$ ("scaling part of the beta function") which we show (in Sect 7) to be zero.

3) We deduce from 1),2) an expansion in powers of $\left\{\underline{r}_{h}\right\}_{h<0}$, convergent if $\left|\underline{r}_{h}\right| \leq \varepsilon$ for all $h \leq 0$, of the pair (and higher) Schwinger functions The expansion implies, if $\left|\underline{r}_{h}\right| \leq \varepsilon$ for all $h \leq 0$, that the pair Schwinger function approaches 0 as its argument $\xi \rightarrow \infty$ faster than the free Schwinger function does, and we compute exactly how' fast (i e we compute the anomaly exponent).

Some support to the validity of the vanishing of $\beta(\underline{r})$ in 2) above was given in [BG, BGM], by reducing it to the proof of a similar conjecture for the Luttinger model. In [BGM] the proof of the conjecture was reduced to a property of the Schwinger functions which is implied by the results in 3) above, plus the independence of the exact solubility of the Luttinger model from the cutoffs necessary to define it. Thus we showed that the exact solubility of the latter model would allow us to establish a rigorous proof of the conjecture if we know suitable uniformity properties on the Luttinger model running constants defined in a way entirely analogous to the one followed for our problem, see [BGM].

The above scheme of proof is discussed in Sect. 7 and, using the new results derived in the previous sections (3-6), it is completed.

The discussion of 1) requires the solution of two distinct problems. The first is an ultraviolet problem, which could be considered trivial. We perform it in detail, but we find no unexpected difficulty (Sect. 3). The second problem is the infrared problem. From the power counting point of view this is just a renormalizable problem with a double singularity (the singular locus of the propagator being reduced to two points). Therefore at first sight it looks technically similar to the ultraviolet stability of the Gross Neveu model in two dimensions, [FMRS, GK1]. However the infrared problem in our case presents new difficulties since it is not asymptotically free. Its solution requires the discussion of an anomalous dimension (physically this means that the perturbed system has correlations which decay at $\infty$ faster than the free ones). To establish rigorously the theory of the corresponding anomalous renormalization group flow is a major technical difficulty that we meet in this work.

Other references on rigorous renormalization group and anomalous dimensions are $[\mathrm{GK} 2, \mathrm{~F}, \mathrm{BG} 1, \mathrm{G}, \mathrm{dV}]$. The first two, however, deal with a deeply different 
notion; namely the anomaly is not dynamically defined, but it is rather a priori supposed to be a part of the free theory, as the free propagator is assumed to have a scaling different from the "normal" and one finds that it remains unchanged. The last reference, $[\mathrm{dV}]$, contains a treatment of an ultraviolet problem with infinite wave function renormalization, but the paper does not seem to discuss the anomalous scaling of the correlation functions at small distances. In this paper the analogous infrared problem (the anomalous scaling at large distances) requires considerable supplementary work.

Interesting technical developments to treat infrared problems with finite wave function renormalization, which go quite far beyond the first work on it [GK3], can be found in [O']. The main difficulty with a dynamical anomaly is that the wave function renormalization is infinite and that this affects the correlations in a rather involved way.

The formalism to deal with a "normal" free propagator and an "anomalous" interacting one is set up in [BG1], (see also [G]), following the original work of [WF], where the anomaly is a fixed (but "dynamical") constant characteristic of the non-trivial point, and following the interpretation of [WF] that Felder provided us (private communication). In our case the anomaly is "dynamical," (and the wave function renormalization is infinite), but it is a function of the coupling, as we do not have one isolated non-trivial fixed point but, instead, a "line of fixed points": this causes a few extra difficulties.

To our knowledge this is the first example of a rigorous theory of the beta function of an anomalous renormalization group flow with a rigorous discussion of its consequences for the anomalous asymptotic behaviour of the correlation functions; and, technically, the correlation functions behaviour represents the major part of this work.

The results of Sect. 7 also imply the existence of a one parameter family of nontrivial fixed points of our renormalization group transformation. This can be regarded as the origin of the anomalous dimension; however we only allude (Sect. 5) to such a corollary as it is not essential for our work.

In the next section we set up the formalism in a self consistent way trying to discuss the rigorous issues growing out of the functional integral representation of the Schwinger functions that we plan to use in the rest of the paper.

It is useful to state our main result in a form independent on the subsidiary concepts (like running coupling, beta function, etc.) and based solely on the hamiltonian (1.3) and on the standard notion of pair Schwinger function, $S(x)$, of the model (introduced formally in the next section); it can be summarized in the following theorem:

Theorem. Given a pair potential $\lambda \bar{v}(\vec{x}-\vec{y})$, with $\bar{v}$ smooth and with short range $p_{0}^{-1}$, one can find analytic functions $\alpha(\lambda), \nu(\lambda)$, holomorphic near $\lambda=0$ and of order $\lambda$, such that the one dimensional spinless Fermi gas with hamiltonian:

$$
\sum_{i=1}^{N}\left(\frac{-\Delta_{\vec{x}_{2}}}{2 m(\lambda)}-\frac{p_{F}^{2}}{2 m(\lambda)}+\nu(\lambda)\right)+2 \lambda \sum_{i<j} \bar{v}\left(\vec{x}_{\imath}-\vec{x}_{\jmath}\right)
$$

with $m(\lambda)=[1+\alpha(\lambda)]^{-1} m, m>0$, admits a zero temperature Gibbs state (defined as the $T \rightarrow 0$ limit of a $T>0$ Gibbs state) with a Euclidean pair Schwinger function $S(x-y)$ verifying, for $|x-y| p_{0}$ large, the relation.

$$
S(x-y)=\frac{S_{0}(x-y)}{\left(p_{0}|x-y|\right)^{2 \eta(\lambda)}}+A(\lambda) \frac{1}{\left(p_{0}|x-y|\right)^{1+2 \eta(\lambda)}}
$$


with $\eta(\lambda), A(\lambda)$ anayltic near $\lambda=0, \eta(\lambda)=O\left(\lambda^{2}\right)$ independent on $x, y, A(\lambda)=O(\lambda)$ (uniformly dependent on $x, y$ ), and with $S_{0}$ being the pair Schwinger function for the free gas with Fermi momentum $p_{F}$ and mass $m$.

Note that $S_{0}(x-y)$ tends to zero with oscillations on scale $p_{F}^{-1}$ and speed $|x-y|^{-1}$, so that the first term in (1.11) dominates over the second "when non-zero."

The theorem was proposed by Tomonaga who developed theoretical argument for its validity, [T]; on the basis of Tomonaga's work Luttinger proposed a model which, if Tomonaga's ideas were correct, should behave in the same way as the system (1.1) that we are considering, [L]. The model differs from (1.1) in two respects: first there are two spinless particles, and second the kinetic energy is linear in the momentum. Luttinger also gave arguments to suggest that the model might be exactly soluble. The model was solved exactly, later, by Mattis and Lieb, proving that indeed it did behave as expected on the basis of its heuristic equivalence to Tomonaga's theory of the model (1.1), [ML].

For higher dimensional many fermions systems a formalism parallel to ours, also based on renormalization group analysis near the Fermi surface has been developed in [FT1, FT2, FMR] with emphasis on the BCS theory. In particular a constructive result similar to 1), (analyticity of the beta functional) but stated in a different language has been proven in [FMRT] for many fermions in two dimensions.

\section{Functional Integral Representation of Fermionic Correlation Functions}

The Schwinger functions of a Hamiltonian $H$ like (1.3) are defined by:

$$
S\left(\vec{x}_{1}, t_{1}, \sigma_{1}, \ldots, \vec{x}_{s}, t_{s}, \sigma_{s}\right)=\frac{\operatorname{Tr}\left(e^{-\left(\beta-t_{1}\right) H} \psi_{\vec{x}_{1}}^{\sigma_{1}} \cdot e^{-\left(t_{s-1}-t_{s}\right) H} \psi_{\vec{x}_{s}}^{\sigma_{s}} e^{-t_{s} H}\right)}{\operatorname{Tr} e^{-\beta H}}
$$

for $\beta>t_{1}>t_{2}>\ldots>t_{s}>0, \psi_{\vec{x}}^{\sigma}, \sigma= \pm$, being field operators on the Fock space of a fermion system confined in a box of size $L$, with periodic boundary conditions, and at temperature $\beta^{-1}>0$.

At fixed $\beta, L$ the (2.1) are, by inspection, real analytic in $\lambda, \alpha, \nu$ : their holomorphy domain has complex size which, for the time being, is totally out of control and it may shrink to 0 as $\beta \rightarrow \infty$ or $L \rightarrow \infty$.

If we are willing to take $\lambda, \nu, \alpha$ of $O\left(\eta L^{-1}\right)$ with $\eta$ small, it is not difficult to see that we have in fact uniformity in $\beta$ as $\beta \rightarrow \infty$. The basic reason is that, if $\lambda, \nu, \alpha$ are so small, we see by perturbation theory that the lowest eigenvalues of $H$ is separated by a gap from the next. Hence the limit as $\beta \rightarrow \infty$ is simply expressed in terms of the expectation value in the ground state $|0\rangle_{\lambda, \nu, \alpha}$ (which is also analytic in such small $\lambda, \nu, \alpha)$, as:

$$
S\left(\vec{x}_{1}, t_{1}, \sigma_{1}, \ldots, \vec{x}_{s}, t_{s}, \sigma_{s}\right)={ }_{\lambda, \nu, \alpha}\left\langle 0\left|\psi_{\vec{x}_{1}}^{\sigma_{1}} \ldots e^{-\left(t_{s-1}-t_{s}\right) H} \psi_{\vec{x}_{s}}^{\sigma_{s}}\right| 0\right\rangle_{\lambda, \nu, \alpha} .
$$

This is manifestly analytic in $\lambda, \nu, \alpha$. Knowing the above analyticity property we can find the expansion coefficients in powers of $\lambda, \nu, \alpha$. The classical calculation is as follows.

We define the imaginary time fields [see (1.4)] as:

$$
\psi_{\vec{x}, t}^{ \pm}=L^{-1 / 2} \sum_{\vec{k}} e^{ \pm i \vec{k} \vec{x} \pm e(k) t} a_{\vec{k}}^{ \pm} \equiv e^{t T} \psi_{\vec{x}}^{ \pm} e^{-t T}
$$


Then by using the representation [where $V \equiv \bar{V}+\nu N+\alpha T$, see (1.3)]:

$$
e^{-t H}=\lim _{n \rightarrow \infty}\left(e^{-t T / n}\left(1-\frac{t V}{n}\right)\right)^{n}
$$

we find that the numerator of (2.1) becomes:

$$
\begin{aligned}
\sum & \pm \int \operatorname{Tr}\left\{e^{-\beta T} V\left(t_{1}^{\prime}\right) \ldots V\left(t_{p_{1}-1}^{\prime}\right)\right. \\
& \left.\times \psi_{\vec{x}_{1}, t_{p_{1}}^{\prime}}^{\sigma_{1}} \ldots \psi_{\vec{x}_{s}, t_{p_{1}+}^{\prime}+p_{s}}^{\sigma_{s}} \ldots V\left(t_{p_{1}+}^{\prime}+p_{s+1}\right)\right\} d \underline{t}^{\prime}
\end{aligned}
$$

where $V(t)=e^{t T} V e^{-t T}$ and the sum runs over integers $p_{1}, p_{2}, \ldots$ while the integral is over all the $t_{j}^{\prime}$ variables with $j \neq p_{1}, p_{1}+p_{2}, \ldots, p_{1}+p_{2}+\ldots+p_{s}$ and $t_{p_{1}}^{\prime}, t_{p_{1}+p_{2}}^{\prime}, \ldots, t_{p_{1}+p_{2}+}^{\prime}+p_{s}$ are fixed to be $t_{1}>t_{2}>\ldots>t_{s} \geq 0$, respectively; finally the $t^{\prime}$ variables are constrained to decrease in their index $j$, and the sign \pm is + if the number of $V$ factors is even and - otherwise.

Since the product of $V^{\prime}$ s is an integral of a sum of products of $\psi_{\vec{x}, t}^{ \pm}$operators and since the $T$ is a quadratic hamiltonian in the $\psi^{ \pm}$operators, Wick's theorem holds for evaluating $\operatorname{Tr}(\exp -\beta T(\cdot)) / \operatorname{Tr}(\exp -\beta T)$ (see, for example, [NO]) and therefore it will be possible to express the various terms in (2.5) as suitable integrals of sums of products of expressions like:

$$
\begin{aligned}
& g_{+}(\vec{\xi}, \tau)=\operatorname{Tr} e^{-\beta T} \psi_{\vec{x}, t}^{-} \psi_{\vec{x}^{\prime}, t^{\prime}}^{+} / \operatorname{Tr} e^{-\beta T}, \\
& g_{-}(\vec{\xi}, \tau)=\operatorname{Tr} e^{-\beta T} \psi_{\vec{x}, t}^{+} \psi_{\vec{x}^{\prime}, t^{\prime}}^{-} / \operatorname{Tr} e^{-\beta T},
\end{aligned}
$$

if $\vec{\xi}=\vec{x}-\vec{x}^{\prime}, \tau=t-t^{\prime}>0$, which we combine to form a single function:

$$
g(\vec{\xi}, \tau)=\left\{\begin{array}{ll}
g_{+}(\vec{\xi}, \tau) & \text { if } \tau>0 \\
-g_{-}(-\vec{\xi},-\tau) & \text { if } \tau \leq 0
\end{array} .\right.
$$

Then it is easy to see, from Wick's theorem, that the generic term in (2.5) can be expressed graphically as follows.

One lays down graph elements like:

Fig. 1.
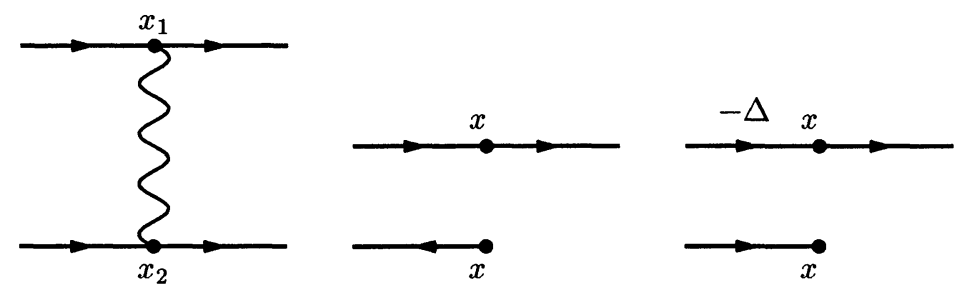

symbolizing respectively:

$$
\begin{aligned}
& -\lambda \bar{v}\left(\vec{x}_{1}-\vec{x}_{2}\right) \psi_{\vec{x}_{1}, t}^{+} \psi_{\vec{x}_{2}, t}^{+} \psi_{\vec{x}_{2}, t}^{-} \psi_{\vec{x}_{1}, t}^{-} \\
& -(\nu-\mu \alpha) \psi_{\vec{x}, t}^{+} \psi_{\vec{x}, t}^{-} \\
& (\alpha / 2 m) \psi_{\vec{x}, t}^{+}(-\Delta) \psi_{\vec{x}, t}^{-} \\
& \psi_{\vec{x}, t}^{+} \quad \text { and } \quad \psi_{\vec{x}, t}^{-} .
\end{aligned}
$$


One should then draw $n+s$ such elements so that the first $n$ have a shape of one of the first three forms with labels $\left(\vec{y}_{i}, t_{i}\right)$ attached arbitrarily to the vertices ("free labels") and the last $s$ have a shape of the last two forms (representing respectively $\psi_{\vec{x}, t}^{-}$or $\left.\psi_{\vec{x}, t}^{+}\right)$and carry "external labels" $\left(\vec{x}_{1}, t_{1}\right), \ldots,\left(\vec{x}_{s}, t_{s}\right)$.

Then one considers all Feynman graphs, that is all possible ways of joining together lines in pairs so that no unpaired line is left over and so that only lines with consistent orientations are allowed to form a pair.

To each graph we assign a sign $\sigma= \pm$ obtained by considering the permutation necessary to bring next to each other the pairs of operators which, in the given graph, are paired (one says also contracted), with the $\psi^{-}$to the left of the associated $\psi^{+}$, and then setting $\sigma=(-1)^{\pi}$ if $\pi$ is the permutation parity.

To each graph we assign a value which is the integral over the free vertices of the product of the sign factor times the product of factors $g(\vec{\xi}, \tau)$ (or of some of its derivatives) for every line with an arrow pointing from $\left(\vec{x}_{1}, t_{1}\right)$ to $\left(\vec{x}_{2}, t_{2}\right)$ with $\vec{\xi}=\left(\bar{x}_{2}-\bar{x}_{1}\right), \tau=t_{2}-t_{1}$, times a factor $-\lambda \bar{v}\left(\vec{x}_{1}-\vec{x}_{2}\right)$ for every wiggly line joining $\left(\vec{x}_{1}, t\right)$ to $\left(\vec{x}_{2}, t\right)$, times a factor $-(\nu-\mu \alpha)$ or $-\alpha / 2 m$ for every vertex of the type with only two lines.

The propagator function $g$ is given by (2.7) and can be represented as:

$$
g(\vec{\xi}, \tau)=L^{-1} \sum_{\vec{k}} e^{-i \vec{k} \vec{\xi}}\left\{\frac{e^{-\tau e(\vec{k})}}{1+e^{-\beta e(\vec{k})}} \chi(\tau>0)-\frac{e^{-(\beta+\tau) e(\vec{k})}}{1+e^{-\beta e(\vec{k})}} \chi(\tau \leq 0)\right\},
$$

where $\chi($ "condition") $=1$ if "condition" is verified and $\chi=0$ otherwise.

This can be written:

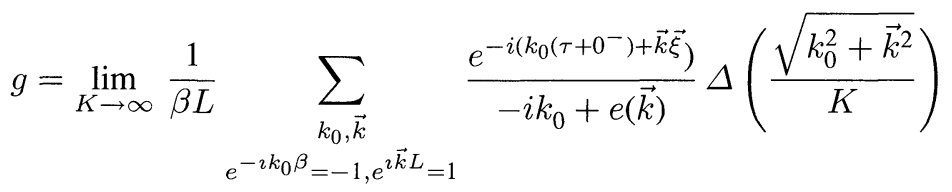

with the sum running over the $k_{0}, \vec{k}$ verifying $e^{-i k_{0} \beta}=-1, e^{-i \vec{k} L}=+1$; and $\Delta$ is a cutoff function like one of the following:

$$
\Delta_{s}(x)=\chi(x<1), \quad \Delta_{\alpha}(x)=\left(1+\frac{x^{2}}{\alpha}\right)^{-\alpha}, \quad \Delta_{\infty}(x)=e^{-x^{2}} .
$$

The (2.10) can be proved, in the case of the first regularization $\Delta=\Delta_{s}$ ("sharp momentum regularization"), by remarking that, if $\tau>0$ and $\vec{k}$ is fixed in the r.h.s. of (2.10), the sum over $k_{0}$ has a limit, for $K \rightarrow \infty$, equal to:

$$
\frac{1}{2 \pi} \oint \frac{e^{-i z \tau}}{(-i z+e(\vec{k}))\left(1+e^{-\imath z \beta}\right)} d z
$$

with the contour running parallel to the real axis (nearer than $|e(\vec{k})| \geq e_{\min } \sim \frac{p_{F}}{m} \frac{\pi}{L}$ ) and going from $-\infty$ to $+\infty$ if $\operatorname{Im} z<0$ and from $+\infty$ to $-\infty$ if $\operatorname{Im} z>0$. Using that $\beta>\tau>0$ we easily see that (2.10) implies (2.7). If $\tau<0$ (but $\beta>|\tau|$ so that $\beta+\tau>0$ ) we see from (2.10) that the sum has value -1 times the value when $\tau$ is replaced by $\beta+\tau$ (because $e^{i \beta k_{0}} \equiv-1$ ). Hence for such values of $\tau$ the value of $g$ is given by -1 times the value of (2.12) with $\tau$ replaced by $\beta+\tau$, and (2.9) follows also for $\tau<0$. 
The cutoff $\Delta_{\alpha}$ can be treated in the same way (if $\alpha=$ positive integer as we suppose): one finds instead of (2.10) a complex integral that can be, essentially, explicitly evaluated and one can therefore estimate easily the difference between (2.10) and (2.9) as $K \rightarrow \infty$.

The gaussian cut-off $\Delta_{\infty}(x)$ cannot be treated by using complex integrals because $\Delta_{\infty}$ has bad behaviour at $\pm i \infty$. But $\Delta_{\infty}(x)-\Delta_{\alpha}(x)=O\left(x^{4}\right)$ as $x \rightarrow 0$ and this, together with the fact that we know that (2.10) holds with the regularization $\Delta_{\alpha}$, easily implies the validity of (2.10) with the gaussian cut-off as well.

Therefore we can compute the coefficients of the perturbation theory for the Schwinger functions by the above graphical algorithms and by using propagators with one of the above cut-offs and then removing it.

The above discussion suggests the following definition:

Definition. Suppose that, for $\lambda$ in a small neighbourhood $D$ of the origin in the complex plane, and for $\alpha, \nu$ suitably chosen as analytic functions of $\lambda$ in $D$, the perturbation series for the Schwinger functions can be shown to admit an analytic continuation to the domain $D$, extending on the real axis to $\lambda$ 's of $O(1)$, i.e. $\beta$, $L$-independent, and suppose that the limits as $\beta \rightarrow \infty, L \rightarrow \infty$ of the Schwinger functions exist in $D$. Then we say that the limit as $L, \beta \rightarrow \infty$ of the Schwinger functions defines a Gibbs state for our system with Fermi momentum $p_{F}$ and particles mass $m+O(\lambda)$ if the asymptotic relation (1.11) holds for some $\eta(\lambda)$ and for all $\lambda$ small enough.

Note that such a definition would certainly not be adequate for $d=3$ (because changing the sign of $\lambda$ destroys the stability of the Hamiltonian, see [R, Th], and the system collapses) and probably not even for $d=2$ (although in this case the sign of $\lambda$ does not affect stability, if $\lambda$ is small enough). Hence, for $d>1$, we would replace the requirement that $D$ is a neighbourhood of the origin by the requirement that it is a domain in the right half plane.

This shows that one can conceive a purely perturbative approach to the low temperature Fermi systems. One starts with some expressions of the perturbation expansion for the Schwinger functions depending on various parameters to be eventually sent to $\infty$ (e.g. $\beta$ or $L$ or others that will be introduced later). At fixed values of the parameters the expansions should be obviously convergent for small $\lambda, \alpha, \nu$. Then one proves uniform analyticity in a region $D$ of complex $\lambda$, where $\alpha, \nu$ are suitably chosen as a function of $\lambda$ (analytic in the same domain) and thus one defines, by removing the cutoffs, a Gibbs state in the above sense.

As long as other cut-offs, besides $\beta, L$, are removed first, the already remarked and obvious analyticity in $\lambda, \alpha, \nu$ at fixed $\beta, L$ guarantees that the functions obtained in this way do have the required positivity property necessary to interpret them as Schwinger functions for a Gibbs state (namely the reflection positivity). In fact the series expansions for real $\lambda, \alpha, \nu$ must coincide with the non-perturbative definitions of the same expressions by analyticity and the latter, of course, have the reflection positivity property.

The most convenient representation of the Schwinger functions, for the above purposes, is the Euclidean functional integral representations. Such a representation is set up with the help of two extra regularization parameters that we call $R, U$, with $R \leq U$, and of a family of Grassmannian variables.

Here the Grassmannian variables will be denoted $A_{k \omega}^{h \sigma}, \varepsilon_{k}^{\sigma}$ and they bear labels $h \in \mathbf{Z}, \omega= \pm 1, \sigma= \pm 1, k=\left(k_{0}, \vec{k}\right)$ such that:

$$
e^{-i k_{0} \beta}=-1, \quad e^{i \vec{k} L}=+1
$$


They must verify anticommutation rules:

$$
\left\{A, A^{\prime}\right\}=0, \quad\{A, \varepsilon\}=0, \quad\left\{\varepsilon, \varepsilon^{\prime}\right\}=0 .
$$

It is most convenient to think of the $A, \varepsilon$ as concrete objects by using a representation on a Hilbert space $\mathbf{h}$. The best Hilbert space is probably the countable tensor product of two dimensional spaces $\mathbf{C}^{2}: \mathbf{h}=\bigotimes_{j=1}^{\infty} \mathbf{C}^{2}$. Then we order (absolutely arbitrarily) the variables labels, by replacing each of them with an integer label $j=1,2, \ldots$ and set the $j^{\text {th }}$ Grassmannian variable to be:

$$
\left[\bigotimes_{i<j} \sigma_{i}^{z}\right] \otimes \sigma_{j}^{+},
$$

where $\sigma^{z}, \sigma^{+}$are the usual Pauli matrices.

Hence the Grassmannian variables can be regarded as a set of creation operators (just creation and no annihilation) on a Fock space.

The $A, \varepsilon$ variables are norm 1 operators on $\mathbf{h}$. They will be used to define the euclidean field on scale $h$ and the external field $\varphi_{x}^{\sigma}$ as

$$
\begin{aligned}
\psi_{x \omega}^{h \sigma} & =\sum_{k} \frac{e^{i \sigma\left(k x-p_{F} \omega \vec{x}\right)}}{\sqrt{\beta L}} \frac{\left(e^{-\gamma^{-2 h} \beta(k)}-e^{-\gamma^{-2 h+2} \beta(k)}\right)^{1 / 2}}{\sqrt{-i k_{0}+e(\vec{k})}} \sqrt{\chi\left(\omega \gamma^{-h} \vec{k}\right)} A_{k \omega}^{h \sigma}, \\
\varphi_{x}^{\sigma} & =\sum_{k} e^{i \sigma k x} \varepsilon_{k}^{\sigma},
\end{aligned}
$$

where $x=(\vec{x}, t), \beta(k)=\left[k_{0}^{2}+e(\vec{k})^{2}\right] p_{0}^{-2}$, and $\chi(t)=\pi^{-1 / 2} \int_{-\infty}^{t} d s \exp \left(-s^{2}\right)$ is a regularization of the step function. The motivation of such a definition will be explained in the following sections.

We define the fields with ultraviolet cut off on scale $\gamma^{-U}$ and infrared on scale $\gamma^{-R}$ as:

$$
\psi_{x \omega}^{[R, U] \sigma}=\sum_{h=R}^{U} \psi_{x \omega}^{h \sigma}
$$

The fields $\psi_{x \omega}^{[R, U] \sigma}$ are bounded operators on $\mathbf{h}$, because the $A$ 's have norm 1 .

The fields $\psi_{x \omega}^{[R, U] \sigma}$ and $\psi_{x \omega}^{h \sigma}$ are quasi-particle fields. We also define the particle fields simply by:

$$
\psi_{x}^{h \sigma}=\sum_{\omega} e^{i \sigma p_{F} \omega \vec{x}} \psi_{x \omega}^{h \sigma}, \quad \psi_{x}^{[R, U] \sigma}=\sum_{\omega} e^{i \sigma p_{F} \omega \vec{x}} \psi_{x \omega}^{[R, U] \sigma}
$$

As a matter of fact, we shall not introduce the quasi-particle fields and we shall introduce the cut-off in a different way, in the ultraviolet region $h>0$; therefore the particle fields will be defined in terms of suitable $A_{k}^{h \sigma}$ variables in a different way. However, in order to simplify the notation, we nevertheless proceed in a symmetric way in the ultraviolet and infrared region; it will be clear that our definitions would work also for the representation of the field used in the following sections.

The Grassmannian or fermionic functional integral is then defined as a linear functional on the operators on $\mathbf{h}$, in the algebra generated by the Grassmannian 
variables. The integration rule is simply the Wick rule based on the following "propagator":

$$
\int A_{k \omega}^{h-} A_{k^{\prime} \omega^{\prime}}^{h^{\prime}+} d P=\delta_{h h^{\prime}} \delta_{k k^{\prime}} \delta_{\omega \omega^{\prime}}
$$

while all the integrals of $A^{+} A^{+}$and $A^{-} A^{-}$vanish. This means that the integral of an arbitrary monomial in the $A^{+}$and $A^{-}$is obtained as a sum over the pairings of the factors into pairs with non-zero propagator of the product of the propagators corresponding to the pairs times a sign \pm equal to the parity of the permutation necessary to bring the considered pairs next to each other.

The above rule is just a linear functional and we may have problems in the integration of expressions which are not finite linear combination of products of $A$ : but of course this is precisely the kind of operation that we shall wish to do.

Therefore it is convenient to define a class of operators on $\mathbf{h}$ on which we can operate the functional integral "absent-mindedly." It will be the class of integrable operators.

Definition. An operator $O(\psi, \varphi)$ is said to be integrable if it has the form:

$$
\begin{aligned}
O(\psi, \varphi)= & \sum_{n, m, \underline{\omega}, \underline{\omega}^{\prime}} \int\left(\prod_{i=1}^{n} d x_{i} d y_{i}\right)\left(\prod_{j=1}^{m} d u_{j} d v_{j}\right) O_{n, m}\left(\underline{x}, \underline{y}, \underline{u}, \underline{v}, \underline{\omega}, \underline{\omega}^{\prime}\right) \\
& \times\left[\mathscr{V}_{1} \psi_{u_{1} \omega_{1}}^{+} \ldots \mathscr{D}_{2 m} \psi_{v_{m} \omega_{m}^{\prime}}^{-}\right] \varphi_{x_{1}}^{+} \ldots \varphi_{y_{n}}^{-}
\end{aligned}
$$

where the $O_{n, m}(\ldots)$ are the "kernels of $O(\psi, \varphi)$ " and $\psi^{ \pm}$are quasi-particle operators on various scales between two scales $R, U$, for all $n$, and $\mathscr{D}_{1} \ldots \mathscr{D}_{2 m}$ are differential operators with constant coefficients (possibly dependent on $h, \omega$ ), and with order bounded by some $N$, for all $n$. Furthermore the $O_{n, m}$ should be measures (i.e. $\delta$ functions are allowed) and:

$$
\begin{aligned}
|O(\psi, \varphi)|_{b} \equiv & \sum_{n, m, \underline{\omega}, \underline{\omega}^{\prime}} b^{n+m} \int\left(\prod_{i=1}^{n} d x_{\imath} d y_{\imath}\right)\left(\prod_{\jmath=1}^{m} d u_{j} d v_{j}\right) \\
& \times\left|O_{n, m}\left(\underline{x}, \underline{y}, \underline{u}, \underline{v}, \underline{\omega}, \underline{\omega}^{\prime}\right)\right|<\infty \quad \forall b>0 .
\end{aligned}
$$

Then we define [consistently with (2.19), as it is possible to check]:

$$
\begin{aligned}
\int P(d \psi) O(\psi, \varphi)= & \sum_{n, m, \omega, \omega^{\prime}} \int\left(\prod_{i=1}^{n} d x_{i} d y_{i}\right)\left(\prod_{j=1}^{m} d u_{j} d v_{j}\right) O_{n, m}\left(\underline{x}, \underline{y}, \underline{u}, \underline{v}, \underline{\omega}, \underline{\omega}^{\prime}\right) \\
& \times \mathscr{D}_{1} \mathscr{D}_{2} \ldots \mathscr{D}_{2 m} \operatorname{det}\left[g_{\omega_{i} \omega_{j}^{\prime}}^{[R, U]}\left(u_{i}-v_{j}\right)\right] \varphi_{x_{1}}^{+} \ldots \varphi_{y_{n}}^{-},
\end{aligned}
$$

where the propagator $g^{[R, U]}(x-y)$ is $\sum_{h=R}^{U} g^{(h)}(x-y)$, with:

$$
\begin{aligned}
g_{\omega \omega^{\prime}}^{(h)}(x-y)= & \frac{\delta_{\omega \omega^{\prime}}}{\beta L} \sum_{k} e^{-i\left[k(x-y)-p_{F} \omega(\vec{x}-\vec{y})\right]} \\
& \times \frac{e^{-\gamma^{-2 h} \beta(k)}-e^{-\gamma^{-2 h+2} \beta(k)}}{-i k_{0}+e(\vec{k})} \chi\left(\omega \gamma^{-h} \vec{k}\right) .
\end{aligned}
$$


Remark. The r.h.s. of (2.22) is a well defined operator, thanks to (2.21), as a consequence of the Gramm-Hadamard inequality (see Appendix 2):

$$
\left|\mathscr{D}_{1} \ldots \mathscr{V}_{2 m} \operatorname{det}\left[g_{\omega_{i} \omega_{j}^{\prime}}^{[R, U]}\left(u_{\imath}-v_{j}\right)\right]\right| \leq B_{R, U}^{m}
$$

Furthermore the definition is meaningful since the representation (2.20) is unique if the kernels:

$$
\mathscr{D}_{1} \mathscr{D}_{2} \ldots \mathscr{D}_{2 m} O_{n, m}\left(\underline{x}, \underline{y}, \underline{u}, \underline{v}, \underline{\omega}, \underline{\omega}^{\prime}\right)
$$

are antisymmetric in the permutation between themselves of the $\left(u_{\imath}, \omega_{\imath}\right)$, of the $\left(v_{i}, \omega_{i}^{\prime}\right)$, of the $x_{i}$ and of the $y_{i}$.

Several easy theorems follow. For instance, if $O$ is integrable also $\exp O$ is integrable: this is a key property that overcompensates the fact that the fermionic integration is not a positive functional in the sense of measure theory (and makes the world of fermionic integration look like a fairy tale compared to that of measure theory.)

Also, if $O(\psi, \varphi)$ is integrable and if we write $\psi^{[R, U]}=\psi_{1}+\psi_{2}$ with $\psi_{1}=\psi^{\left[R, U_{1}\right]}$ and $\psi_{2}=\psi^{\left[U_{1}, U\right]}$, then $O\left(\psi_{1}+\psi_{2}, \varphi\right)=\sum O_{1}\left(\psi_{1}, \varphi\right) O_{2}\left(\psi_{2}, \varphi\right)$ and $O_{\imath}$ are integrable; moreover:

$$
\int P(d \psi) O(\psi, \varphi)=\sum \int P\left(d \psi_{1}\right) O_{1}\left(\psi_{1}, \varphi\right) \int P\left(d \psi_{2}\right) O_{2}\left(\psi_{2}, \varphi\right)
$$

i.e. "Fubini's theorem" holds.

The above obvious remarks constitute the theory of non-commutative or fermionic Grassmannian integration.

Its interest lies in the fact that it is easy to see that the coefficients of the perturbation expansion of the Schwinger functions are generated by:

$$
q_{R, U}(\varphi)=\log \int P\left(d \varphi^{[R, U]}\right) e^{-V(\psi)+\int d x\left(\varphi_{x}^{+} \psi_{x}^{-}+\psi_{x}^{+} \varphi_{x}^{-}\right)}
$$

via:

$$
S^{T}\left(x_{1}, \sigma_{1}, \ldots, x_{n}, \sigma_{n}\right)=\left.\lim _{\substack{U \rightarrow \infty \\ R \rightarrow-\infty}} \frac{\delta^{2 n} q_{R, U}(\varphi)}{\delta \varphi_{x_{1}}^{+} \ldots \delta \varphi_{y_{n}}^{-}}\right|_{\varphi=0}
$$

Hence we shall confine ourselves to studying $q_{R, U}(\varphi)$ and reorganizing the expansion of $S^{T}$ in powers of $\lambda$ (with $\nu, \alpha$ also expanded in terms of $\lambda$ ) so that the expansion have analyticity properties in $\lambda$ uniform in $R, U$ as well as in $L, \beta$. We shall also use the expansion to infer the long distance behaviour of $S^{T}\left(x_{1}, \sigma_{1}, \ldots, x_{n}, \sigma_{n}\right)$ [long means $O(L)$ in space and $O(\beta)$ in time].

Remark. $q_{R, U}(\varphi)$ has an expression like (2.20) (with $n=0$ ), whose kernels are the functional derivatives appearing in the r.h.s. of (2.28). Furthermore one can define the $\left|q_{R, U}(\varphi)\right|_{b}$ norm as in (2.21) and it is possible to see [using (2.24) and some standard procedure to bound the truncated expectations, see last part of Appendix 3] that is norm is finite for $b \leq b_{0}$, with $b_{0}$ depending on the strength of the interaction; this is sufficient to define $q_{R, U}(\varphi)$ as a bounded operator.

In order to simplify the notation, in the following sections we shall consider, for the propagator, only the limiting case $L=\beta=\infty$, by interpreting the functional integrals as a formal tool to represent in a convenient way the expansions of the Schwinger functions in powers of $\lambda, \alpha, \nu$. It will be clear that all our results are valid 
also for $L, \beta$ finite and that one can take the limit $L, \beta \rightarrow \infty$ without any further problem.

Moreover we shall change the meaning of the symbol $\psi_{x}^{\sigma}$ [see (2.3)], which from now on will denote the formal limit $R \rightarrow-\infty, U \rightarrow+\infty$ of the Grassmannian field $\psi_{x}^{[R, U] \sigma}$ defined in (2.18). Then we can write the generating functional of the Schwinger functions, in the limit where all the cut off are removed, as:

$$
q(\varphi)=\log \int P(d \psi) e^{-V(\psi)+\int d x\left(\varphi_{x}^{+} \psi_{x}^{-}+\psi_{x}^{+} \varphi_{x}^{-}\right)},
$$

and we can say that $P(d \psi)$ is Grassmannian gaussian measure with propagator:

$$
g(x-y)=\int P(d \psi) \psi_{x}^{-} \psi_{y}^{+}=\int \frac{d k_{0} d \vec{k}}{(2 \pi)^{2}} \frac{e^{-i\left[k_{0}\left(\left(x_{0}-y_{0}\right)+0^{-}\right)+\vec{k}(\vec{x}-\vec{y})\right]}}{-i k_{0}+e(\vec{k})},
$$

where the $0^{-}$in the exponential means that $g(0, \vec{x})$ must be interpreted as

$$
\lim _{x_{0} \rightarrow 0^{-}} g\left(x_{0}, \vec{x}\right) \text {. }
$$

Moreover, if $\Lambda=L \times[0, \beta]$ :

$$
\begin{aligned}
V(\psi)= & \lambda \int_{\Lambda \times \Lambda} d x d y v(x-y) \psi_{x}^{+} \psi_{y}^{+} \psi_{y}^{-} \psi_{x}^{-} \\
& +(\nu-\mu \alpha) \int_{\Lambda} d x \psi_{x}^{+} \psi_{x}^{-}+\alpha \int_{\Lambda} d x \psi_{x}^{+}(-\Delta) \psi_{x}^{-} \\
v(x-y) \equiv & \delta\left(x_{0}-y_{0}\right) \bar{v}(\vec{x}-\vec{y}),
\end{aligned}
$$

where $\Delta=\partial_{\vec{x}}^{2}$ is the Laplacian in the space variables.

A very convenient object which is related to $q(\varphi)$ is the effective potential defined by:

$$
e^{-V_{\mathrm{eff}}(\varphi)}=\frac{1}{\mathscr{N}} \int P(d \psi) e^{-V(\psi+\varphi)},
$$

where $\mathscr{N}^{\prime}$ is a normalization constant chosen so that $V_{\text {eff }}(0)=0$.

The relation is, if $(g \varphi)^{-}=g * \varphi^{-}$and $(g \varphi)^{+}=\varphi^{+} * g^{\prime}$, where the $*$ denotes convolution and $g^{\prime}(x)=g(-x)$, the following:

$$
-V_{\mathrm{eff}}(g \varphi)+\left(\varphi^{+}, g \varphi^{-}\right)=q(\varphi) \text {. }
$$

The above relations are formally trivial if one treats $\int P(d \psi)$. as an ordinary integral with respect to a Grassmannian measure proportional to:

$$
d \psi^{+} d \psi^{-} e^{-\int\left[\psi_{x}^{+}\left(\partial_{t}+\left(-\Delta+p_{F}^{2}\right) / 2 m\right] \psi_{x}^{-} d x\right.},
$$

and proceeding to the change of variables $\psi+g \varphi=\tilde{\psi}$.

The formal argument on the change of variables is meaningless as presented; however if one writes the above calculations (i.e. the change of variables) as relations between the power series in the fermion fields defining the fermionic integrals, one sees that they are indeed valid.

Equation (2.33) should allow us, in principle, to reduce the study of the Schwinger functions to that of the effective potential. However, because of the anomalous large distance behaviour, this is not so simple, in the sense that it is not possible to use 
directly (2.33), see [BGM]. In any event, the analysis of the effective potential will play an essential role; therefore, in the following three sections, we shall analyze the integral in the r.h.s. of (2.32) by an iterative procedure, based on the scale decomposition (2.17) of the field. This will allow us to define the effective potential on scale $\gamma^{-h}$, whose properties will be used in Sect. 6 to study the pair Schwinger function, by an expansion that will take the place of the relation (2.33). The same technique could be used also to study the other Schwinger functions, but we shall not do it explicitly.

\section{Ultraviolet Limit for the Effective Potential}

In this section we shall begin the analysis of the effective potential defined in Eq. (2.32), by studying the ultraviolet problem.

We start by decomposing $g(x)$ in its u.v. (ultraviolet) and its i.r. (infrared) part:

$$
g(x)=g_{\mathrm{u} \mathrm{v}}(x)+g_{\mathrm{ir}}(x)
$$

with

$$
g_{\mathrm{u} \mathrm{v}}(x)=\int \frac{d k_{0} d \vec{k}}{(2 \pi)^{2}} \frac{1-e^{-\left(k_{0}^{2}+e(\vec{k})^{2}\right) p_{0}^{-2}}}{-i k_{0}+e(\vec{k})} e^{-i\left(k_{0}\left(x_{0}+0^{-}\right)+\vec{k} \vec{x}\right)},
$$

where $p_{0}^{-1}$ is the range of the potential, see (1.1) and (3.24) below.

It is easy to see that:

$$
g(x)=\theta\left(x_{0}\right) e^{\frac{x_{0} p_{F}^{2}}{2 m}}\left(\frac{m}{2 \pi x_{0}}\right)^{1 / 2} e^{-\frac{m \vec{x}^{2}}{2 x_{0}}}-\int_{-p_{F}}^{p_{F}} \frac{d \vec{k}}{2 \pi} e^{-i \vec{k} \vec{x}-x_{0} \frac{\vec{k}^{2}-p_{F}^{2}}{2 m}}
$$

where $\theta\left(x_{0}\right)$ is the step function. Hence we can write:

$$
g_{\mathrm{u} \mathrm{v}}(x)=G(x)+R(x)
$$

with:

$$
\begin{aligned}
G(x)= & h(\vec{x}) h\left(x_{0}\right) \theta\left(x_{0}\right) e^{\frac{x_{0} p_{F}^{2}}{2 m}}\left(\frac{m}{2 \pi x_{0}}\right)^{1 / 2} e^{-\frac{m \vec{x}^{2}}{2 x_{0}}}, \\
R(x)= & {\left[1-h(\vec{x}) h\left(x_{0}\right)\right] g_{\mathrm{u} \mathrm{v}}(x)-h(\vec{x}) h\left(x_{0}\right) g_{\mathrm{i} \mathrm{r}}(x) } \\
& -h(\vec{x}) h\left(x_{0}\right) \int_{-p_{F}}^{p_{F}} \frac{d \vec{k}}{2 \pi} e^{-i \vec{k} \vec{x}-x_{0} \frac{\vec{k}^{2}-p_{F}^{2}}{2 m}},
\end{aligned}
$$

where $h(t), t \in \mathbf{R}^{1}$, is a smooth function of compact support such that $h(t)=1$, if $|t| \leq 1$, and $h(t)=0$, if $|t| \geq \gamma, \gamma$ being any number greater than 1 , fixed once and for all. $A, \bar{\kappa}$ :

It is easy to show that $R(x)$ is a smooth function on $\mathbf{R}^{2}$, such that, for suitable

$$
|R(x)| \leq A e^{-\bar{\kappa}|x|} .
$$

Equations (3.1), (3.4), and (2.32) imply that:

$$
e^{-V_{\text {eff }}(\varphi)}=\frac{\overline{\mathscr{N}}^{(0)}}{\mathscr{N}} \int P^{(\mathrm{i} \mathrm{r})}\left(d \psi^{(\mathrm{i} \mathrm{r})}\right) e^{-\bar{V}^{(0)}\left(\psi^{(\mathrm{i} \mathrm{r})}+\varphi\right)},
$$


where

$$
e^{-\bar{V}^{(0)}(\varphi)}=\frac{\mathscr{N}^{(0)}}{\overline{\mathscr{N}}^{(0)}} \int P^{(R)}(d \psi) e^{-V^{(0)}(\psi+\varphi)}
$$

and

$$
e^{-V^{(0)}(\varphi)}=\frac{1}{\mathscr{N}^{(0)}} \int P^{(G)}(d \psi) e^{-V(\psi+\varphi)}
$$

with $\mathscr{N}^{(0)}, \overline{\mathscr{N}}^{(0)}$ defined so that $V^{(0)}(0)=\bar{V}^{(0)}(0)=0$, and $P^{(\mathrm{i} \mathrm{r})}(d \psi), P^{(R)}(d \psi)$, $P^{(G)}(d \psi)$ are the Grassmannian integrations with propagator $g_{\mathrm{i}}(x), R(x)$, and $G(x)$, respectively.

In order to give a meaning to (3.10), we now introduce an u.v. cutoff by replacing $G$ with:

$$
G_{N}(x)=\theta_{N}\left(x_{0}\right) h(\vec{x}) e^{\frac{x_{0} p_{F}^{2}}{2 m}}\left(\frac{m}{2 \pi x_{0}}\right)^{d / 2} e^{-\frac{m \vec{x}^{2}}{2 x_{0}}}
$$

where $\theta_{N}(t)$ is a smooth function with support in the interval $\left[\gamma^{-N}, \gamma\right]$ and $N$ is a large positive integer.

Note that the cut-off is different from that introduced in Sect. 2, which has allowed us to present in a symmetric way the ultraviolet and the infrared problems. However, one can check that the results of this section do not depend on the choice of the cut-off; in fact, one could add the new cut-off to the previous one, parametrized by $U$, and note that all bounds are uniform in $U$. Furthermore, in this section we shall use only the particle field representation of the Grassmannian integrations, see (2.18).

It is convenient to define more precisely $\theta_{N}(t)$ in the following way:

$$
\theta_{N}(t)=\sum_{i=1}^{N} f\left(\gamma^{i} t\right)
$$

where:

$$
f(t)=[h(t / \gamma)-h(t)] \theta(t)
$$

is a smooth function with support on $\left[1, \gamma^{2}\right]$. The function $\theta_{N}(t)$ has the claimed support properties and:

$$
\theta(t) h(t)=\lim _{N \rightarrow \infty} \theta_{N}(t) .
$$

It is worth remarking that:

$$
\lim _{N \rightarrow \infty} G_{N}(x)=G(x), \quad \text { for all } x \in \mathbf{R}^{2},
$$

because in the discontinuity point $x_{0}=0$, by definition,

$$
G\left(0, x_{1}\right)=\lim _{x_{0} \rightarrow 0^{-}} G\left(x_{0}, x_{1}\right)=0=G_{N}\left(0, x_{1}\right) .
$$

Two other consequences immediately follow from (3.15):

1 ) in (3.10) we can suppose that the potential (2.31) is Wick ordered w.r.t. $G_{N}$, since only products of fields at coinciding times appear in it;

2) all Feynman graphs with closed fermion loops in the perturbative expansion of $V^{(0)}(\varphi)$ vanish; furthermore, because of the $\delta\left(x_{0}-y_{0}\right)$ in (2.31), also the loops containing some lines $v(x-y)$ are forbidden, if the directions of the fermionic lines are compatible. 
Then we define:

$$
V^{(0)}(\varphi)=\lim _{N \rightarrow \infty} \log \frac{1}{\mathscr{N}^{(0)}} \int P^{(\leq N)}\left(d \psi^{(\leq N)}\right) e^{-V\left(\psi^{(\leq N)}+\varphi\right)},
$$

where $P^{(\leq N)}\left(d \psi^{(\leq N)}\right)$ is the Grassmannian integration with propagator $G_{N}$.

We want to prove that the limit exists and that it is an analytic function of $z=(\lambda, \nu, \alpha)$ in a neighbourhood of $z=0$, in the sense that the kernels $O_{n}$ of the operator $O=V^{(0)}(\varphi)$, defined as in (2.20) (without the sum over $\omega, \omega^{\prime}$ ), are analytic functions verifying, in their holomorphy domain, bounds like (2.21). We shall also prove that $V^{(0)}(\varphi)$ has some "exponential decay" properties (i.e., its kernels decay exponentially fast as the arguments separate to $\infty$ ). The extension of these results to $\bar{V}^{(0)}(\varphi)$ will be trivial. More precisely we shall prove the following theorem:

Theorem 1. There exist $\varepsilon>0$ and $D>0$ such that $\bar{V}^{(0)}(\varphi)$ can be written, for $|z| \leq \varepsilon$, if $z=(\alpha, \nu, \lambda)$, in the following way:

$$
\begin{aligned}
\bar{V}^{(0)}(\psi)= & \lambda \int d x d y v(x-y) \psi_{x}^{+} \psi_{y}^{+} \psi_{y}^{-} \psi_{x}^{-}+2 \lambda \int d x d y v(x-y) R(x-y) \psi_{x}^{+} \psi_{y}^{-} \\
& +(\nu-4 \pi \lambda \hat{v}(0) R(0)) \int d x \psi_{x}^{+} \psi_{x}^{-}+\alpha \int d x \psi_{x}^{+}\left(\frac{-\Delta-p_{F}^{2}}{2 m}\right) \psi_{x}^{-} \\
& +\int d x d y \psi_{x}^{+} \Delta \psi_{y}^{-} \tilde{W}_{2}(z, x-y) \\
& +\sum_{n=1}^{\infty} \sum_{n_{1}, n_{2}} \int d x_{1} \ldots d x_{2 n} \psi_{x_{1}}^{+} \ldots \psi_{x_{n}}^{+} \psi_{x_{n+1}}^{-} \ldots \psi_{x_{2 n-n_{2}}}^{-} \\
& \times \Delta \psi_{x_{2 n-n_{2}+1}^{-}}^{-} \ldots \Delta \psi_{x_{2 n}}^{-} W_{n_{1} n_{2}}\left(z, x_{1} \ldots x_{2 n}\right)
\end{aligned}
$$

where the kernels $W_{n_{1} n_{2}}$ are products of suitable delta functions by smooth functions, which are analytic in $z$ if $|z| \leq \varepsilon$, and satisfy, uniformly in $N$, the following estimate:

$$
\begin{aligned}
& \left.\int d x_{1} \ldots d x_{2 n}\left|W_{n_{1} n_{2}}\left(z, x_{1} \ldots x_{2 n}\right)\right| e^{\frac{\kappa}{2} d^{(0)}\left(x_{1}\right.} x_{2 n}\right) \\
& \quad \leq|\Lambda|(D|z|)^{\max \{2, n-1\}}
\end{aligned}
$$

while $\tilde{W}_{2}(z, x)$ singles out some "special" contributions (see discussion after (3.40) below) and satisfies (uniformly in $N$ ):

$$
\begin{gathered}
\int d x\left|\tilde{W}_{2}(z, x)\right||x| e^{\frac{\kappa}{2}|x|} \leq(D|z|)^{2}, \\
\int d x \tilde{W}_{2}(z, x)=0 .
\end{gathered}
$$

The r.h s of (3.18) is summable in $n$, for $|z|$ small enough and we shall take this property as definition of analyticity round $z=0$ for a function of the field of the general form (3.17), see also Sect. 2, (2.20), (2.21).

We shall study the integral in (3.16) by decomposing the Grassmannian integration $P^{(\leq N)}\left(d \psi^{(\leq N)}\right)$ in the product of the independent integrations $P^{(h)}\left(d \psi^{(h)}\right), h=$ 
Fig. 2.

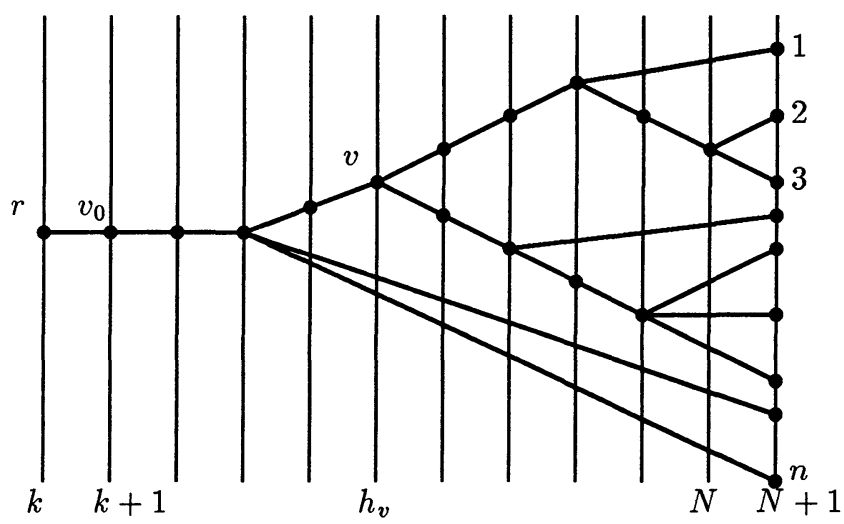

$1, \ldots, N$, with propagator:

$$
C_{h}(x)=f\left(\gamma^{h} x_{0}\right) h(\vec{x}) e^{\frac{x_{0} p_{F}^{2}}{2 m}}\left(\frac{m}{2 \pi x_{0}}\right)^{1 / 2} e^{-\frac{m \vec{x}^{2}}{2 x_{0}}}=\gamma^{h / 2} \bar{C}_{h}\left(\gamma^{h} x_{0}, \gamma^{h / 2} \vec{x}\right),
$$

where $\bar{C}_{h}(x)$ is a smooth function such that, for suitable $A$ and $\bar{\kappa}$ :

$$
\left|\bar{C}_{h}(x)\right| \leq A e^{-\bar{\kappa}|x|}, \quad \forall h \geq 1,
$$

and $\bar{\kappa}$ can be taken to be the same as in (3.7). In fact, by (3.12)

$$
G_{N}(x)=\sum_{h=1}^{N} C_{h}(x) .
$$

We shall assume that $A$ is chosen so that also the following bound is satisfied:

$$
|\bar{v}(\vec{x}-\vec{y})| \leq A e^{-p_{0}|\vec{x}-\vec{y}|}
$$

for a suitable $p_{0}$; we shall call $p_{0}^{-1}$ the range of the potential $\vec{v}$, see (1.1).

We shall integrate iteratively the fields $\psi^{(h)}$ in (3.16), by studying the properties of the effective potential on scale $\gamma^{-k}$, defined by:

$$
\begin{aligned}
V^{(k)}(\varphi)= & \lim _{N \rightarrow \infty} \log \frac{1}{\mathscr{N}^{(k)}} \int P^{(k+1)}\left(d \psi^{(k+1)}\right) \ldots P^{(N)}\left(d \psi^{(N)}\right) \\
& \times e^{-V\left(\psi^{(k+1)}+\psi^{(N)}+\varphi\right)}
\end{aligned}
$$

so that:

$$
e^{-V^{(0)}(\varphi)}=\frac{\mathscr{N}^{\cdot(k)}}{\mathscr{N}^{(0)}} \int P^{(\leq k)}\left(d \psi^{(\leq k)}\right) e^{-V^{(k)}\left(\psi^{(\leq k)}+\varphi\right)} .
$$

An essential role in our analysis will be played by the tree expansion (see [G]), with which we assume that the reader is familiar. We start with some definitions and notations.

1) Let us consider the family of all trees which can be constructed by joining a point $r$, the root, with an ordered set of $n \geq 1$ points, the endpoints of the unlabeled tree (see Fig. 2). Two unlabeled trees that can be superposed by a suitable continuous 
deformation, so that the endpoints with the same index coincide, will be said to have the same topological structure and they will be regarded as equivalent.

The unlabeled trees are partially ordered from the root to the endpoints in the natural way (we shall use the symbol $<$ to denote the order); $n$ will be called the order of the unlabeled tree.

We shall consider also the labeled tree (which in general will be simply called trees in the following); they are defined by associating some labels with the unlabeled trees, as explained in the following items. We shall denote $\mathscr{T}_{n}$ the set of labeled trees of order $n$.

2) Given $\tau \in \mathscr{T}_{n}$, we associate with each endpoint one of the three terms of (2.31), which we denote $\tilde{V}_{\alpha}, \alpha$ being a suitable label, and which we represent pictorially by the following graph elements:

Fig. 3.
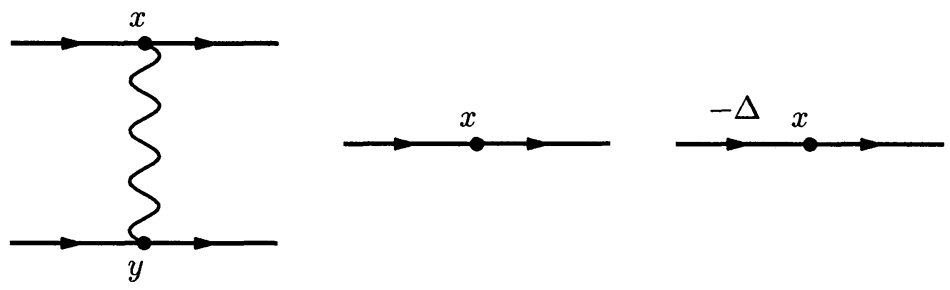

We shall say that the three different graph elements are of type $4,2,2^{\prime}$, respectively, and we shall call space vertices the corresponding integration variables (a more appropriate name would be "space inverse-temperature vertices," but this is too long). 3) We introduce a family of vertical lines, labeled by a frequency index $h$, which takes all the integer values between $k$ and $N+1$; the vertical lines are ordered from left to right as the frequency index increases. Furthermore the root of the labeled tree must belong to the line with index $k$, the endpoints must belong to the line index $N+1$ and, finally, any branch point must belong to a vertical line with index larger than $k$ and smaller than $N+1$.

We call non-trivial vertices of $\tau$ its branch points (this set is empty if $n=1$ and, in this case, there is only one unlabeled tree); we call trivial vertices the points where the branches connecting two non-trivial vertices intersect the family of vertical lines; finally, we call vertices the trivial or non-trivial vertices and the endpoints (see the dots in Fig. 2). Note that there are no vertices on the endbranches of the tree except the endpoints.

Given a vertex $v$, we denote $h_{v}$ the frequency index of the vertical line containing it; note that:

$$
h_{v^{\prime}}<h_{v}, \text { if } v^{\prime}<v \text {. }
$$

The first vertex of the tree (having frequency index $k+1$ ) will be denoted $v_{0}$. Given a trivial or non-trivial vertex $v, s_{v}$ will denote the number of lines branching from $v$ (then $s_{v}=1$, if $v$ is a trivial vertex).

4) We can associate to the endpoints $4 n_{4}+2 n_{2}+2 n_{2}^{\prime}=n_{r}$ fields, if the number of different graph elements associated with the tree is, respectively, $n_{4}, n_{2}, n_{2}^{\prime}$; we shall attach a label $f=1, \ldots, n_{\tau}$ to each field to distinguish them.

5) We shall denote $x_{2 i-1}, x_{2 i}, i=1, \ldots, n_{4}$, the $2 n_{4}$ space vertices associated with the $n_{4}$ graphs elements of type 4 and $x_{2 n_{4}+j}, j=1, \ldots, n-n_{4}$, those associated with 
the graph elements of type 2 and $2^{\prime} ; \underline{x}$ will be the set of all space vertices. We shall use also the notation $x_{j}=\left(t_{j}, \vec{x}_{j}\right)$ for the time and space components of $x_{j}$.

Note that each $x_{j}$ is associated with a pair of fields $\psi_{x_{j}}^{+}$and $\psi_{x_{j}}^{-}$or $\psi_{x_{j}}^{+}$and $\Delta \psi_{x_{j}}^{-}$ and, conversely, to every field of label $f$, representing a line of a graph element, corresponds a point $x_{(f)}$, representing the vertex of the graph element from which it emerges, and a label $\sigma_{f}$, allowing to distinguish the three possibilities $\psi_{x_{\jmath}}^{+}, \psi_{x_{j}}^{-}$, and $\Delta \psi_{x_{j}}^{-}$.

6) Finally, we denote $\mathscr{E}_{h}$ and $\mathscr{E}_{h}^{T}$ the expectation and the truncated expectation, respectively, with respect to the Grassmannian integration $P^{(h)}\left(d \psi^{(h)}\right)$.

As it is explained in [G], we can express the contribution to the effective potential on scale $\gamma^{-k}$ of order $n$ in the $z$ variables, say $V^{(k)}\left(N, n, \psi^{(\leq k)}+\varphi\right)$, in the following way (setting $\varphi=0$ for simplicity of notation):

$$
V^{(k)}\left(N, n, \psi^{(\leq k)}\right)=\sum_{\tau \in \mathbb{F}_{n}} V^{(k)}\left(N, \tau, \psi^{(\leq k)}\right),
$$

where:

$$
V^{(k)}\left(N, \tau, \psi^{(\leq k)}\right)=\left[\prod_{v} \frac{1}{s_{v} !} \mathscr{E}_{h_{v}}^{T}\right] \prod_{i=1}^{n}\left[\mathscr{E}_{h_{i}+1} \ldots \mathscr{E}_{N}\left(\tilde{V}_{\alpha_{\imath}}\left(\psi^{(\leq N)}\right)\right)\right] .
$$

The tree definition and the corresponding combinatorial weights in (3.28) are adopted from $[\mathrm{FG}]$, rather than from [GN, G].

By remark 1) after (3.15), the last expectations in the r.h.s. of (3.28) have the only effect of lowering the cutoff of the field to $h_{i}=h_{v_{\imath}}$ if $v_{\imath}$ is the top non-trivial vertex out of which emerge the line representing $\tilde{V}_{\alpha_{\imath}}\left(\psi^{(\leq N)}\right)$ (this is in fact one of the main properties of the Wick ordering); therefore we can write:

$$
V^{(k)}\left(N, \tau, \psi^{(\leq k)}\right)=\left[\prod_{v} \frac{1}{s_{v} !} \mathscr{E}_{h_{v}}^{T}\right]\left[\prod_{i=1}^{n} \tilde{V}_{\alpha_{\imath}}\left(\psi^{\left(\leq h_{\imath}\right)}\right)\right]
$$

This is a symbolic notation; the $\mathscr{E}^{T}$ operations have to be thought of as performed hierarchically starting from the higher non-trivial vertices and going down along the tree toward the root.

We need to find a more explicit expression for the r.h.s. of (3.29). We begin by proving that $V^{(k)}\left(N, n, \psi^{(\leq k)}\right)$ can be also written in the following way:

$$
V^{(k)}\left(N, n, \psi^{(\leq k)}\right)=\int d \underline{x}^{\left(P_{v_{0}}\right)} \sum_{\tau \in \mathscr{T}_{n}} \sum_{P_{v_{0}}} W^{(k)}\left(N, \tau, P_{v_{0}}, \underline{x}^{\left(P_{v_{0}}\right)}\right) \tilde{\psi}^{(\leq k)}\left(P_{v_{0}}\right)
$$

where:

$$
W^{(k)}\left(N, \tau, P_{v_{0}}, \underline{x}^{\left(P_{v_{0}}\right)}\right)=\int V^{(k)}\left(N, \tau, P_{v_{0}}, \underline{x}\right) d\left(\underline{x} \backslash \underline{x}^{\left(P_{v_{0}}\right)}\right) .
$$

$P_{v_{0}}$ is a non-empty subset of $I_{v_{0}}=\left\{1, . ., n_{\tau}\right\}$, the field labels associated with the tree endpoints reachable from $v_{0}$ (i.e. all of them); the sum $\sum_{P_{v_{0}}}$ is the sum over such 
subsets and $\underline{x}^{\left(P_{v_{0}}\right)}$ is the set of space vertices from which the fields labeled by $P_{v_{0}}$ emerge. Finally:

$$
\tilde{\psi}^{(\leq k)}\left(P_{v_{0}}\right)=\prod_{f \in P_{v_{0}}} \psi_{x_{(f)}^{\sigma_{f}(\leq k)}}
$$

and the kernels $V^{(k)}(\cdot)$ in (3.31) are suitably chosen. Note that in (3.32) there is no Wick order: it is in fact convenient to work without it.

It is convenient to think of $I_{v_{0}}$ as an ordered set and of $P_{v_{0}}$ as an ordered subset: in this way it will be easier to keep track of the sign changes due to the anticommutation relations between the Grassmannian variables.

If $\tau$ has no non-trivial vertex, (3.30) is trivial; hence we can proceed by induction, by assuming that (3.30) is true for all subtrees of $\tau$ branching from $v_{0}$ (if $s_{v_{0}}=1$, there is only one subtree). By using (3.29), we can write:

$$
\begin{aligned}
& \sum_{P_{v_{0}}} V^{(k)}\left(N, \tau, P_{v_{0}}, \underline{x}\right) \tilde{\psi}^{(\leq k)}\left(P_{v_{0}}\right) \\
& =\frac{1}{s_{v_{0}} !} \sum_{P_{v_{0}^{1}},,_{v_{v_{0}}}} \prod_{j=1}^{s_{v_{0}}}\left(V^{(k+1)}\left(N, \tau_{\jmath}, P_{v_{0}^{\jmath}}, \underline{x}_{\jmath}\right)\right) . \\
& \quad \times \mathscr{E}_{k+1}^{T}\left(\tilde{\psi}^{(\leq k+1)}\left(P_{v_{0}^{1}}\right), \ldots, \tilde{\psi}^{(\leq k+1)}\left(P_{v_{0}} s_{v_{0}}\right)\right),
\end{aligned}
$$

where:

1) $\tau_{j}, j=1, \ldots, s_{v_{0}}$, are the $s_{v_{0}}$ subtrees with root in $v_{0}$, whose frequency index is $k+1$

2) $n_{j}$ is the degree of the tree $\tau_{j}$, (with $\left.\sum_{j} n_{\jmath}=n\right)$;

3) $P_{v_{0}^{j}}$ is a non-empty subset of the set $I_{v_{0}^{j}}$ of the $n_{\tau_{j}}$ fields in $\tau_{j}$;

4) $v_{0}^{1}, \ldots, v_{0}^{s_{v_{0}}}$ are the vertices immediately following $v_{0}$ in the tree.

Now we use the identity:

$$
\tilde{\psi}^{(\leq k+1)}(P)=\sum_{Q \subseteq P}(-1)^{\Pi} \tilde{\psi}^{(k+1)}(P \backslash Q) \tilde{\psi}^{(<k+1)}(Q),
$$

where $\Pi$ is the parity of the permutation necessary to pass from the ordered subset $P$ to the ordered subset obtained by writing first $Q$ followed by $P \backslash Q$; and $P \backslash Q$ denotes the complement of $Q$ in $P$. We can write:

$$
\begin{aligned}
(3.33)= & \frac{1}{s_{v_{0}} !} \sum_{P_{v_{0}^{1}}, P_{v_{0}}} \prod_{j=1}^{s_{v_{0}}}\left(V^{(k+1)}\left(N, \tau_{j}, P_{v_{0}^{j}}, \underline{x}_{j}\right)\right) \\
& \times \sum_{Q_{v_{0}^{1}}, Q_{v_{0}} s_{v_{0}}} \mathscr{E}_{k+1}^{T}\left(\tilde{\psi}^{(k+1)}\left(P_{v_{0}^{1}} \backslash Q_{v_{0}^{1}}\right), \ldots,\right. \\
& \left.\times \tilde{\psi}^{(k+1)}\left(P_{v_{0} s_{0}} \backslash Q_{v_{0}}^{s_{v_{0}}}\right)\right) \tilde{\psi}^{(\leq k)}\left(P_{v_{0}}\right),
\end{aligned}
$$

where $P_{v_{0}}=\bigcup_{i} Q_{v_{0}^{2}}$ is the set of fields which are not contracted and $Q_{v_{0}^{2}} \subset P_{v_{0}^{2}}$. 
The latter relation proves our assumption (3.30) with:

$$
\begin{aligned}
& V^{(k)}\left(N, \tau, P_{v_{0}}, \underline{x}\right)=\frac{1}{s_{v_{0}} !} \sum_{P_{v_{0}^{1}},} \sum_{, P_{v_{0}}} \prod_{j=1}^{s v_{0}}\left(V^{(k+1)}\left(N, \tau_{j}, P_{v_{0}^{\jmath}}, \underline{x}_{j}\right)\right) \\
& \quad \times \mathscr{E}_{k+1}^{T}\left(\tilde{\psi}^{(k+1)}\left(P_{v_{0}^{1}} \backslash Q_{v_{0}^{1}}\right), \ldots, \tilde{\psi}^{(k+1)}\left(P_{v_{0}} s_{v_{0}} \backslash Q_{v_{0}} s_{v_{0}}\right)\right),
\end{aligned}
$$

where $Q_{v_{0}^{i}}=P_{v_{0}^{i}} \cap P_{v_{0}}$.

By using the expression (3.36) iteratively it is easy to find the general expression for $V\left(N, \tau, P_{v_{0}}, \underline{x}\right)$ : one has simply to write explicitly $V^{(j)}\left(N, \tau_{\jmath}, P_{v_{0}^{j}}, \underline{x}_{\jmath}\right)$ and so on, reaching finally the endpoints of the tree. This yields:

$$
\begin{aligned}
& V\left(N, \tau, P_{v_{0}}, \underline{x}\right)=\left[\prod_{\substack{v>v_{0} \\
n \text { not e p }}} \sum_{P_{v}}\right] \\
& \quad \times\left\{\prod_{v \text { not e p }} \frac{1}{s_{v} !} \mathscr{E}_{h_{v}}^{T}\left(\tilde{\psi}^{\left(h_{v}\right)}\left(P_{v^{1}} \backslash Q_{v^{1}}\right), \ldots, \tilde{\psi}^{\left(h_{v}\right)}\left(P_{v^{s} v} \backslash Q_{v^{s_{v}}}\right)\right)\right. \\
& \left.\quad \times(-\alpha)^{n_{2}^{\prime}}(-\nu)^{n_{2}} \prod_{\imath=1}^{n_{4}}\left[-\lambda v\left(x_{2 i-1}-x_{2 i}\right)\right]\right\},
\end{aligned}
$$

where:

1) $v^{1}, \ldots, v^{s v}$ are the vertices immediately following $v$;

2) If $v$ is a trivial or non-trivial vertex $P_{v}=\bigcup_{\jmath} Q_{v^{\jmath}}$ and $Q_{v^{\jmath}}=P_{v} \cap P_{v^{\imath}}$, then $P_{v}$ is a subset of the set $I_{v}$ of $n_{\tau^{(v)}}$ fields in $\tau^{(v)}$; if $v$ is an endpoint of the tree, $P_{v}$ coincides with the set of fields appearing in the corresponding graph element.

If we expanded the expectations in (3.37) by Wick's theorem, we could represent the r.h.s. as a sum of Feynman graphs in the usual way [see, however, comments after (3.44) below]. Such graphs have internal lines with propagator $C_{h_{v}}$ (and we shall say that they have frequency $h_{v}$ ), if they are generated in $v$ by the operation $\mathscr{E}_{h_{v}}^{T}$; the external lines are associated with the fields appearing in $\tilde{\psi}^{(\leq k)}\left(P_{v_{0}}\right)$. Furthermore, if $\mathscr{G}_{\tau}$ is the set of all Feynman graphs associated to $\tau$, given $g \in \mathscr{G}_{\tau}$, it is natural to associate a subgraph $g_{v}$ to the vertex $v$; the internal lines of $g_{v}$ are the lines generated in all vertices $\geq v$, while the external lines are those associated with the fields appearing in $\tilde{\psi}^{\left(\leq h_{v}-1\right)}\left(P_{v}\right)$.

If we insert (3.37) in (3.31), we obtain a rather explicit expression for the kernel $W^{(k)}$. It is an expression that we shall use to prove that the effective potential is an analytic function of $z \equiv(\lambda, \alpha, \nu)$ around $z=0$ (in the sense of the theorem that we are proving), uniformly in $N$, and that it decays exponentially on scale $\gamma^{-k}$, as the distance between the space vertices $\underline{x}^{\left(P_{v_{0}}\right)}$ goes to infinity. This will be the interpretation of the following ultraviolet bound stating that, for all $N, n, \tau, P_{v_{0}}$ :

$$
\int d \underline{x}^{\left(P_{v_{0}}\right)} \chi_{\tau}\left(\underline{x}^{\left(P_{v_{0}}\right)}\right)\left|W^{(k)}\left(N, \tau, P_{v_{0}}, \underline{x}^{\left(P_{v_{0}}\right)}\right)\right| e^{\frac{\kappa}{2} d^{(k)}\left(P_{v_{0}}\right)} \leq(C|z|)^{n}|\Lambda|,
$$




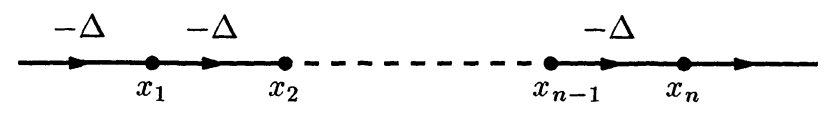

Fig. 4.

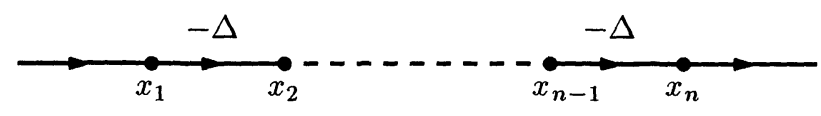

where (here and always in the following) $C$ denotes a suitable positive constant and $\kappa$ is the minimum between $\bar{\kappa}$ and $p_{0}$ [see (3.24), (3.7), (3.22)]; furthermore $d^{(k)}\left(P_{v_{0}}\right)$ and $\chi_{\tau}\left(\underline{x}^{\left(P_{v_{0}}\right)}\right)$ are defined in the following way.

Let $\mathbf{T}$ be the set of all connected tree graphs joining the $m\left(P_{v_{0}}\right)=\left|\underline{x}^{\left(P_{v_{0}}\right)}\right|$ space vertices; if $\mathbf{b} \in \mathbf{T}$, we call $b^{(1)}, \ldots, b^{\left(m\left(P_{v_{0}}\right)-1\right)}$ its bonds and $b_{j}^{(i)}, j=0,1$, the two components of $b^{(i)}(0$ is the index of the time component); then:

$$
d^{(k)}\left(P_{v_{0}}\right) \equiv \min _{b \in T} \sum_{i=1}^{m\left(P_{v_{0}}\right)-1}\left(\gamma^{k}\left|b_{0}^{(i)}\right|+\left|b_{1}^{(i)}\right|\right) .
$$

Let $\mathscr{T}_{n}^{*} \subset \mathscr{T}_{n}$ be the family of trees satisfying one of the following two conditions: a) the graph elements associated with the endpoints of $\tau$ are all of type $2^{\prime}$ and, as a consequence, $\tilde{\psi}^{(\leq k)}\left(P_{v_{0}}\right)=\psi_{x_{1}}^{+(\leq k)} \Delta \psi_{x_{n}}^{-(\leq k)}$;

b) there are $(n-1)$ graph elements of type $2^{\prime}$, while the other one is of type 2 and its $\psi^{-}$line is an external line, so that $\tilde{\psi}^{(\leq k)}\left(P_{v_{0}}\right)=\psi_{x_{1}}^{+(\leq k)} \psi_{x_{n}}^{-(\leq k)}$.

We define:

$$
\chi_{\tau}\left(\underline{x}^{\left(P_{v_{0}}\right)}\right)=\left\{\begin{array}{ll}
\gamma^{k}\left|t_{n}-t_{1}\right|+\gamma^{k / 2}\left|\vec{x}_{n}-\vec{x}_{1}\right| & \text { if } \tau \in \mathscr{T}_{n}^{*} \\
1 & \text { otherwise }
\end{array} .\right.
$$

Note that, if $\tau \in \mathscr{T}_{n}^{*}$, the corresponding graph expansion of

$$
V^{(k)}\left(N, \tau,\left\{h_{v}\right\}, P_{v_{0}}, \underline{x}\right)
$$

contains only chains connecting $x_{1}$ to $x_{n}$, see Fig. 4 .

It is easy to see that their contribution has a singularity, as $\left|x_{1}-x_{n}\right| \rightarrow 0$, whose $L_{1}$ norm is logarithmically divergent when $N \rightarrow \infty$; the $\chi_{\tau}$ factor in (3.38) is introduced to deal with the singularity, (see below).

The contribution to the effective potential of such trees can be easily summed; the result can be expressed in terms of the same two Feynman graphs of Fig. 4, where now the lines represent the full propagator $\sum_{h=k+1}^{N} C_{h}$. Let us consider, for example, the chain of item a) for $k=0$; it is easy to see, by explicit calculation, that such graphs behave, when $\left|x_{1}-x_{n}\right|$ is small, in the limit $N \rightarrow \infty$, as:

$$
\alpha^{n} \vartheta\left(t_{n}-t_{1}\right) \frac{\left(t_{n}-t_{1}\right)^{n-2}}{(n-2) !} \frac{\partial^{n-1}}{\partial t_{n}^{n-1}} e^{-\frac{m\left(\vec{x}_{1}-\vec{x}_{n}\right)^{2}}{2\left(t_{n}-t_{1}\right)}}\left(\frac{m}{t_{n}-t_{1}}\right)^{1 / 2}
$$

which is not $L_{1}$.

The origin of this singularity can be easily understood. Suppose, in fact, that there is an infrared cutoff on scale 1, so that the full propagator coincides with $G(x)$. 
Hence the contribution of the chain to the two points Schwinger function $S_{2}(x-y)$ is obtained by substituting the two external lines with the full propagators $G\left(x-x_{n}\right)$ and $G\left(x_{1}-y\right)$ and one finds that the leading contribution for $|x-y| \rightarrow 0$ behaves as:

$$
\alpha^{n} \vartheta\left(t-t^{\prime}\right) \frac{\left(t-t^{\prime}\right)^{n}}{n !} \frac{\partial^{n}}{\partial t^{n}} e^{-\frac{m(\vec{x}-\vec{y})^{2}}{2\left(t-t^{\prime}\right)}}\left(\frac{m}{t-t^{\prime}}\right)^{1 / 2}
$$

The latter expression can be summed over $n$ and we get a function with the same behaviour of $G(x-y)$ with the substitution $m \rightarrow m /(1+\alpha)$; this result should have been expected, since the term proportional to $\alpha$ in the interaction could be absorbed in the free Grassmannian integration producing exactly such a change in the bare mass of the particles.

The proof of Eq. (3.38) will make use of the fermionic nature of the fields and of the explicit form of the propagator defined in Sect. 2. We shall need the following results for the fermionic expectations:

$$
\begin{aligned}
& \frac{1}{s !}\left|\mathscr{E}_{h}^{T}\left(\tilde{\psi}^{(h)}\left(P_{1}\right), \ldots, \tilde{\psi}^{(h)}\left(P_{s}\right)\right)\right| \\
& \quad \leq \gamma^{\frac{h}{4} \sum_{\jmath}\left|P_{j}^{1}\right|} \gamma^{\frac{5}{4} h \sum_{\jmath}\left|P_{j}^{2}\right|} C^{\sum_{j}\left|P_{\jmath}\right|} \frac{1}{s !} \sum_{T} e^{-\kappa d_{T}^{(h)}\left(P_{1}, \quad, P_{s}\right)}
\end{aligned}
$$

where $|P|=\left|P^{1}\right|+\left|P^{2}\right|$ is the number of elements in $P,\left|P^{1}\right|$ is the number of fields $\left.\psi^{(}\right),\left|P^{2}\right|$ is the number of fields $\Delta \psi^{(~)}$. Furthermore $T$ is an anchored tree graph between the clusters of space vertices from which the fields labeled by $P_{1}, \ldots, P_{s}$ emerge; this means that $T$ is a set of lines connecting two points in different clusters, which becomes a tree graph if one identifies all the points in the same cluster. If $b^{1}, \ldots, b^{s}$ are the lines belonging to $T$ we define:

$$
d_{T}^{(h)}\left(P_{1} \ldots P_{s}\right)=\sum_{j=1}^{s}\left(\gamma^{h}\left|b_{0}^{\jmath}\right|+\gamma^{h / 2}\left|b_{1}^{\jmath}\right|\right) .
$$

Note that, if $s=1$, the sum over $T$ is void and must be understood as a trivial factor 1.

The proof of the bounds (3.43) is in Appendix 2; here we want to stress the absence of factorials in the number of fields, which is essentially linked to the fact that we do not expand 1.h.s. in Feynman graphs

With the aid of (3.43) we can bound (3.37) as follows:

$$
\begin{aligned}
& \left|V^{(k)}\left(N, \tau,\left\{h_{v}\right\}, P_{v_{0}}, \underline{x}\right)\right| \leq\left\{\prod_{\substack{v>v_{0} \\
v \text { not e p }}} \sum_{P_{v}}\right\} \\
& \times \prod_{v \text { not e p }} \prod_{j}\left[\gamma^{\frac{h_{v}}{4}\left[\left|P_{v^{j}}^{1}\right|-\left|Q_{v^{\jmath}}^{1}\right|+5\left|P_{v^{j}}^{2}\right|-5\left|Q_{v^{j}}^{2}\right|\right]}\right] C^{\sum_{j}\left(\left|P_{v^{\jmath}}\right|-\left|Q_{v^{j}}\right|\right)}
\end{aligned}
$$

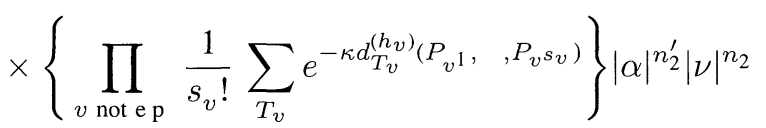

$$
\begin{aligned}
& \times \prod_{\imath=1}^{n_{4}}\left|\lambda v\left(x_{2 \imath-1}-x_{2 i}\right)\right|
\end{aligned}
$$


where $Q_{v^{j}}^{i}=P_{v}^{\imath} \cap P_{v^{j}}^{i}, i=1,2$ and $j=1, \ldots, s_{v}$.

Now we have to integrate the expression (3.45) multiplied by the weight

$$
e^{\frac{\kappa}{2} d^{(k)}\left(P_{v_{0}}\right)}
$$

It is clear that in the r.h.s. of (3.45) $\underline{x}$ appears only in the last line; therefore we have to evaluate the expression:

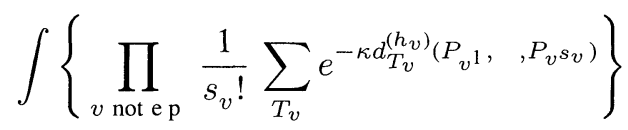

$$
\begin{aligned}
& \times \prod_{i=1}^{n_{4}}\left|\lambda v\left(x_{2 \imath-1}-x_{2 i}\right)\right| e^{\frac{\kappa}{2} d^{(k)}\left(P_{v_{0}}\right)} d \underline{x} .
\end{aligned}
$$

Here we have to use the properties of $v(x-y)$ : in fact a global tree graph (on all the scales) requires in general also the $v$ 's to insure the connection. The property of $v$ that we need is [see (3.24) and (2.31)]:

$$
|\lambda v(x-y)| \leq|\lambda| A e^{-p_{0}|\vec{x}-\vec{y}|} \delta\left(t-t^{\prime}\right)
$$

where $x=(t, \vec{x}), y=\left(t^{\prime}, \vec{y}\right)$.

The latter inequality and a standard estimation of the integral [see Appendix 3, (A3.18)-(A3.20)], allow to bound (3.46) by:

$$
C^{n}|\Lambda| \prod_{v \geq v_{0}} \gamma^{-\frac{3}{2} h_{v}\left(s_{v}-1\right)}|\lambda|^{n_{4}}
$$

By (3.48) and (3.45) we have

$$
\begin{aligned}
& \frac{1}{|\Lambda|} \int d \underline{x}\left|V^{(k)}\left(N, \tau, P_{v_{0}}, \underline{x}\right)\right| e^{\frac{\kappa}{2} d^{(k)}\left(P_{v_{0}}\right)} \\
& \leq C^{n}|\lambda|^{n_{4}}|\alpha|^{n_{2}^{\prime}}|\nu|^{n_{2}}\left\{\prod_{\substack{v>v_{0} \\
v \text { not e p }}} \sum_{P_{v}}\right\} \\
& \times \prod_{v \text { not e p }}\left[\gamma^{\frac{h_{v}}{4}}\left[\sum_{j}\left|P_{v j}^{1}\right|-\left|P_{v}^{1}\right|+5 \sum_{j}\left|P_{v j}^{2}\right|-5\left|P_{v}^{2}\right|\right] \gamma^{-\frac{3}{2} h_{v}\left(s_{v}-1\right)}\right]
\end{aligned}
$$

and we note that:

$$
\begin{aligned}
& \prod_{v \text { not e p }} \gamma^{\frac{h_{v}}{4}\left[\sum_{j}\left|P_{v \jmath}^{1}\right|-\left|P_{v}^{1}\right|+5 \sum_{j}\left|P_{v j}^{2}\right|-5\left|P_{v}^{2}\right|\right]} \\
& \quad=\left[\prod_{v \text { not e p }}\left[\gamma^{\frac{1}{4}\left[6 n_{v}^{2^{\prime}}+4 n_{v}^{4}+2 n_{v}^{2}-\left|P_{v}^{1}\right|-5\left|P_{v}^{2}\right|\right]}\right] \gamma^{\frac{k}{4}\left[6 n_{2^{\prime}}+4 n_{4}+2 n_{2}-\left|P_{v_{0}}^{1}\right|-5\left|P_{v_{0}}^{2}\right|\right]}\right.
\end{aligned}
$$

and:

$$
\prod_{v \text { not e p }} \gamma^{-\frac{3}{2} h_{v}\left(s_{v}-1\right)}=\left[\prod_{v \text { not e p }} \gamma^{-\frac{3}{2}\left(n_{v}-1\right)}\right] \gamma^{-\frac{3}{2} k(n-1)}
$$


Therefore we can rewrite the last factor of 3.49) as:

$$
\left[\prod_{v \text { not e p }} \gamma^{-\frac{1}{4}\left[\left|P_{v}^{1}\right|+5\left|P_{v}^{2}\right|+2 n_{v}^{4}+4 n_{v}^{2}-6\right]}\right] \gamma^{-k / 4\left[2 n_{4}+4 n_{2}+\left|P_{v_{0}}^{1}\right|+5\left|P_{v_{0}}^{2}\right|-6\right]}
$$

where $n_{v}$ is the number of endpoints which follow $v$ in the tree, while $n_{v}^{4}, n_{v}^{2}, n_{v}^{2^{\prime}}$ are the numbers of endpoints of type $4,2,2^{\prime}$ which follow $v$.

Let us observe now that:

$$
\left[\left|P_{v}^{1}\right|+5\left|P_{v}^{2}\right|+2 n_{v}^{4}+4 n_{v}^{2}-6\right]>0
$$

(hence $\geq 1$ ), except in the following cases, that we discuss separately.

1) $\left|P_{v}^{1}\right|=2, n_{v}^{4}=2,\left|P_{v}^{2}\right|=n_{v}^{2}=0$.

The only possibly Feynman graphs associated with $\tau_{v}$ are, in this case:

Fig. 5.
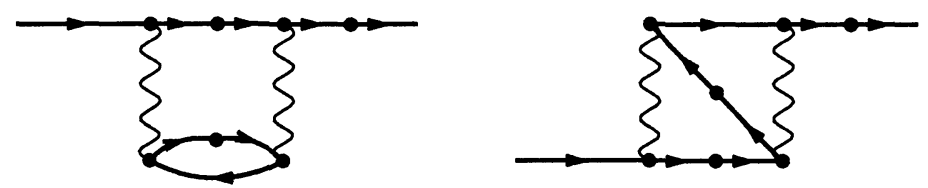

where the dots on the inner lines and on the external outgoing lines represent insertions of type $2^{\prime}$ graph elements. However, their contribution is exactly zero by Remark 2 ) after (3.15), which is valid also for Feynman graphs with propagators of different frequencies.

2) $\left|P_{v}^{1}\right|=2, n_{v}^{4}=1,\left|P_{v}^{2}\right|=n_{v}^{2}=0$.

This is the case of the graphs:

Fig. 6.

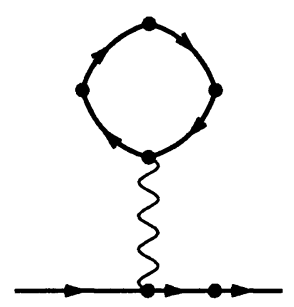

which vanish for the same reason of case 1).

3) $\left|P_{v}^{1}\right|=1,\left|P_{v}^{2}\right|=1, n_{v}^{4}=n_{v}^{2}=0$.

This is the case of the trees, whose graph elements are all of type $2^{\prime}$, so that only the chains of Fig. 7 are allowed.

If $v \neq v_{0}$, one of the two lines external with respect to $v$ is internal to the nontrivial vertex $v^{\prime}$ preceding $v$. To be definite, let us suppose that this is the case for the line emerging from $x_{m}$ (the other case can be treated in the same way); then all terms

Fig. 7.

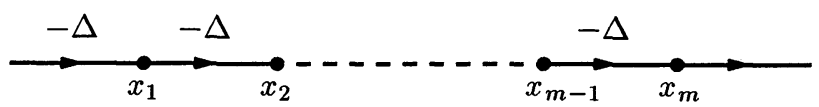


contributing to the expansion in Feynman graphs of $W^{(k)}\left(N, \tau, P_{v_{0}}, \underline{x}^{\left(P_{v_{0}}\right)}\right)$ contain a factor of the type:

$$
\begin{aligned}
& \int d x_{2} \ldots d x_{m} \Delta_{x_{2}} C_{h_{1}}\left(x_{1}-x_{2}\right) \ldots \Delta_{x_{m}} C_{h_{m-1}}\left(x_{m-1}-x_{m}\right) \\
& \left(\Delta_{y}\right)^{\varrho} C_{h_{v^{\prime}}}\left(x_{m}-y\right)
\end{aligned}
$$

where $\varrho=0$ or $\varrho=1$.

Let us suppose first that all the lines have the same frequency, that is $h_{\imath}=h_{v}$, for $i=1, \ldots, m-1$. Then, since $\int d x_{m} \Delta C_{h}\left(x_{m-1}-x_{m}\right)=0$, we can substitute in (3.54) $C_{h_{v^{\prime}}}\left(x_{m}-y\right)$ with:

$$
\begin{aligned}
& C_{h_{v^{\prime}}}\left(x_{m}-y\right)-C_{h_{v^{\prime}}}\left(x_{m-1}-y\right) \\
& \quad=\left(x_{m}-x_{m-1}\right) \int_{0}^{1} d t \partial C_{h_{v^{\prime}}}\left(x_{m}-y-t\left(x_{m}-x_{m-1}\right)\right),
\end{aligned}
$$

and it is easy to see that such a substitution allows us to improve the bound by a factor $\gamma^{-\left(h_{v}-h_{v^{\prime}}\right) / 2}$.

If the lines have different frequencies, i.e. if there are other non-trivial vertices following $v$, we have to apply the previous argument iteratively starting from the higher vertices. The only change is that some covariance in (3.54) is substituted by its gradient calculated at an interpolated point as in the r.h.s. of (3.55); it is easy to see that the improvement for each non-trivial vertex is always the same, i.e. $\gamma^{-1 / 2}$ raised to a power equal to the difference between the frequency of the vertex and that of the preceding non-trivial one. Furthermore, there is at most a factor $\left|x-x^{\prime}\right|$ for each line connecting $x$ and $x^{\prime}$ and each covariance must be interpolated at most two times; so no dangerous factorials appear.

Of course, in order to improve the bound, we have to expand in Feynman graphs the subtree starting in the vertex $v$ and extract the propagator $C_{h_{v^{\prime}}}\left(x_{m}-y\right)$ from the truncated expectation associated with $v^{\prime}$. One could be afraid that this destroys the good combinatorial properties of (3.43), but this is not the case. In fact the subtree starting from $v$ belongs to $\mathscr{T}_{m}^{*}$ and it is easy to see that its expansion in Feynman graphs contains exactly $s_{v}$ ! terms, which is compensated by making use of the $1 / s_{v}$ ! factors of (3.37); so there is no combinatorial problem here. The problem of the extraction of $C_{h_{v^{\prime}}}\left(x_{m}-y\right)$ from the truncated expectation is not really present, since each term contributing to the r.h.s. of (3.43) has a factor equal to one of the external propagators of $v$ [see the proof of (3.43) in Appendix 2].

We have still to consider the case $v=v_{0}$, but now $\tau \in \mathscr{T}_{n}^{*}$ and we can use the factor $\chi_{\tau}\left(\underline{x}^{\left(P_{v_{0}}\right)}\right)$ in (3.38) to improve the bound by a factor $\gamma^{-\left(h_{v}-k\right) / 2}$.

4) $\left|P_{v}^{1}\right|=2,\left|P_{v}^{2}\right|=0, n_{v}^{4}=0, n_{v}^{2}=1$.

This is the case of the tree with an arbitrary number of type $2^{\prime}$ graph elements and one of type 2. The same considerations of Case 3) apply, so that again we can improve the bound by a factor $\gamma^{-\left(h_{v}-h_{v^{\prime}}\right) / 2}$.

We can summarize the discussion above, by saying that the last line of (3.49) can be replaced by expression:

$$
\left[\prod_{v \text { not ep }} \gamma^{-\frac{1}{4} D_{v}} \chi\left(D_{v}>0\right)\right] \gamma^{-\frac{k}{4} D_{v_{0}}}
$$


where $\chi\left(D_{v}>0\right)$ is the characteristic function of the set $\left\{D_{v}>0\right\}$ (it reminds us that the graphs of items 1 and 2 are not allowed) and

$$
\begin{aligned}
D_{v}= & \left|P_{v}^{1}\right|+5\left|P_{v}^{2}\right|+2 n_{v}^{4}+4 n_{v}^{2}-6+2 \delta_{\mid P_{v}^{1 \mid, 1}} \delta_{\left|P_{v}^{2}\right|, 1} \delta_{n_{v}^{4}, 0} \delta_{n_{v}^{2}, 0} \\
& +2 \delta_{\left|P_{v}^{1}\right|, 2} \delta_{\left|P_{v}^{2}\right|, 0} \delta_{n_{v}^{4}, 0} \delta_{n_{v}^{2}, 1} .
\end{aligned}
$$

The above discussion shows the essentially trivial renormalizability of this model. In fact, since the number of unlabeled trees with $n$ endpoints can be bounded by $2^{4 n}$, in order to prove the bound (3.38) it is sufficient to control the multiple sums in (3.49) and the sum over the labeled trees with a fixed topological structure. This can be easily done by using the factors $\gamma^{-\frac{1}{4} D_{v}}$ of (3.56).

We first observe that, given an unlabeled tree $\tilde{\tau}$, there are only $3^{n}$ corresponding families of labeled trees differing for the choice of the graph elements associated with the endpoints; hence it is sufficient to consider only one of such families, say $\tilde{\mathscr{T}}$. Threes $\tau \in \tilde{\mathscr{T}}$ can be distinguished by fixing the frequency indices of the non-trivial vertices, which we shall denote $\tilde{v}$. We can write:

$$
\prod_{v \text { not e p }} \gamma^{-\frac{1}{4} D_{v}} \leq\left[\prod_{\tilde{v}} \gamma^{-\frac{1}{8}\left(h_{\tilde{v}}-h_{\tilde{v}^{\prime}}\right)}\right]\left[\prod_{v \text { not e p }} \gamma^{-\frac{1}{8} D_{v}}\right],
$$

where $\tilde{v}^{\prime}$ is the non-trivial vertex immediately preceding $\tilde{v}$ or the root, if there is no such vertex.

The sum over the set $\tilde{\mathscr{T}}$ of the first factor in the r.h.s. of (3.58) can be bounded in a trivial way by a factor $C^{n}$. Furthermore, by (3.57), if $D_{v}>0$ :

$$
D_{v} \geq \max \left\{1,\left|P_{v}\right|-2\right\} \geq \frac{\left|P_{v}\right|}{3} \text {. }
$$

Hence, in order to complete the proof of (3.38), it is sufficient to prove that:

$$
\prod_{v \text { not e p }} \sum_{P_{v}} \gamma^{-\frac{\left|P_{v}\right|}{24}} \equiv S\left(P_{v_{0}}, \tau, n\right) \leq C^{n}
$$

where the sums over the sets $P_{v}$ are constrained by the condition that $P_{v}=\bigcup_{j} Q_{v^{j}}$ with $Q_{v^{j}}$ a subset, possibly empty, of $P_{v j}$; furthermore $P_{v}$ is a fixed set with four or two elements, if $v$ is an endpoint, and we have eliminated the constraint that $P_{v_{0}}$ is a fixed subset of the fields associated with the tree graph elements.

The latter estimate, evident for large $\gamma$, can be proved in the general case $\gamma>1$ in the following way. We note that:

$$
S\left(P_{v_{0}}, \tau, n\right) \leq \prod_{v \text { not e p }} \sum_{p_{v}} \gamma^{-\frac{p_{v}}{24}} C_{v}
$$

where $C_{v}$ counts the number of ways of choosing a subset $P_{v}$ with $p_{v}$ elements, satisfying the constraints; hence it can be easily bounded by a binomial coefficient and we obtain:

$$
S\left(P_{v_{0}}, \tau, n\right) \leq \prod_{v \text { not e p }} \sum_{p_{v}} \gamma^{-\frac{p_{v}}{24}}\left(\begin{array}{c}
\sum_{j=1}^{s_{v}} p_{v^{\jmath}} \\
p_{v}
\end{array}\right) .
$$


Set $\bar{\gamma}=\gamma^{1 / 24}$ and let us denote with $\mathscr{P}$ a path from the root of the tree to an endpoint and with $l(\mathscr{P})$ the number of vertices lying on $\mathscr{P}$. It is easy to show, by simply performing the sums in (3.62) one after the other starting from $v_{0}$, that:

$$
S\left(P_{v_{0}}, \tau, n\right) \leq \prod_{\tilde{p}}\left(\sum_{n=0}^{l(\mathfrak{p})} \bar{\gamma}^{-n}\right)^{4} \leq\left(\frac{1}{1-\bar{\gamma}^{-1}}\right)^{4 n} .
$$

The bound (3.38) implies that we can sum, for $|z|$ small enough, say $|z| \leq \varepsilon$, and uniformly in $N$, the terms in the effective potential, which have the same dependence on the field (i.e. that have the same set of labels $\left\{\sigma_{f}, f \in P_{v_{0}}\right\}$ ). In fact, we have still to bound only the sum of all trees of order $n$ satisfying that condition: as mentioned above this gives simply another factor $\leq 2^{4 n}$, as the trees are "topological trees," see item 1) after Fig. 2.

We can now integrate also the field fluctuations associated with the regular part $R(x)$ of the u.v. covariance, see (3.4) and (3.9). The regularity of the propagator $R$ makes this a trivial repetition of, say, the last integration lowering the u.v. cut-off from $h=1$ to $h=0$ and we do not have to perform it in detail.

The bounds of this section imply that $\bar{V}^{(0)}(\varphi)$ can be written, for $|z| \leq \varepsilon$, as in (3.17) and that a similar expression is valid for the effective potential on scale $\gamma^{-k}$. Furthermore the kernel $\tilde{W}_{2}(z, x)$ singles out the contributions coming from the trees in $\mathscr{T}_{n}^{*}$ [see discussion after (3.40)] and therefore satisfies (uniformly in $N$ ) the bound (3.19) and Eq. (3.20).

From the considerations of Sect. 2 it is almost obvious that the effective potentials can be given by the expression (3.17) for $|z|<O\left(L^{-1} \gamma^{-N}\right)$. The results just derived show that in fact the analyticity in $z$ of the kernels for the effective potentials can be extended to $|z|<\varepsilon$ for some $\varepsilon>0$ and of order $O(1)$ and have a uniform exponential decay ( $\Lambda, N$-independent): see (3.18), (3.19).

This means that we can sum the coefficients of given order in $z$ and that their sum admits good exponential bounds.

Note that this is not sufficient to guarantee the integrability in the sense of Sect. 2 of $\exp \bar{V}^{(0)}\left(\psi^{(\mathrm{ir})}+\varphi\right)$ with respect to the i.r. part of the Grassmannian fields for $|z|<\varepsilon$.

We shall proceed by imagining that we have a u.v. cut-off $N$ and perform the integrations down to the infrared cut-off $R$ : and we shall see that it is possible to perform a resummation of perturbation theory permitting us to express the effective potentials as uniformly convergent power series in a sequence of constants $\underline{r}_{h}$, called the running couplings, which are themselves expressed as sums of series in the initial couplings $z$. The series for the running couplings will have very small $L, N, R$ dependent radii of convergence. But they will be related by a map permitting us to express their values at scale $h$ in terms of the values at the preceding scales $h-1, \ldots, 0$. We shall show that the relation is expressed by an analytic function, the beta functional, of the preceding couplings with a radius of convergence which is uniform in $N, R, L$. Thus if by some other means, see Sect. 7, one can be sure that the beta functional generates a sequence $\underline{r}_{h}, h=0,-1, \ldots$, of running couplings which stay small uniformly in the index $h$, then one will have shown the possibility of a resummation of the perturbation series for the full effective potential kernels, which is uniform in $R, N, L$ and a theory of the ground state will have been constructed (up to the technicalities analyzed in Sect. 6). 
In the next section we begin the discussion on the beta functional and its analyticity properties.

\section{The Effective Potential in the Infrared Region. Failure of Normal Scaling}

In this section we shall begin the analysis of the infrared problem, that is of the possibility of giving a meaning to the integration in (3.8) of the infrared fluctuations of the field, associated with the propagator:

$$
g_{\mathrm{ir}}(x) \equiv g^{(\leq 0)}(x)=\int \frac{d k_{0} d \vec{k}}{(2 \pi)^{2}} \frac{e^{-\imath k x}}{-i k_{0}+e(\vec{k})} e^{-\left[k_{0}^{2}+e(\vec{k})^{2}\right] p_{0}^{-2}}
$$

Note that the Fourier transform of $g^{(\leq 0)}(x)$ has a linear divergence on the Fermi surface $k_{0}=0, \vec{k}= \pm p_{F}$, which cannot be treated by a naive multiscale decomposition as the one used for the u.v. problem, because of the presence of the built-in scale $p_{F}$. It is possible, however, to rewrite the problem in terms of quasiparticle fields in the way presented in $[\mathrm{BG}]$, that we briefly summarize here.

We write the particle field $\psi_{x}^{\sigma(\leq 0)}$ of covariance $g^{(\leq 0)}(x)$ as a sum of independent quasi-particle fields:

$$
\psi_{x}^{\sigma(\leq 0)} \equiv \sum_{\omega= \pm 1} e^{\imath \sigma p_{F} \omega \vec{x}} \psi_{\omega, x}^{\sigma(\leq 0)}
$$

and, as usual, the fields $\psi_{\omega, x}^{\sigma(\leq 0)}$, essentially describing the fluctuations around the two points of the Fermi surface, are decomposed as sums of independent fields in the following way:

$$
\psi_{\omega, x}^{\sigma(\leq 0)}=\sum_{h=-\infty}^{0} \psi_{\omega, x}^{\sigma(h)}
$$

where $\psi_{\omega, x}^{\sigma(h)}$ has covariance:

$$
\begin{aligned}
g_{\omega}^{(h)}(x)= & e^{\imath p_{F} \omega \vec{x}} \int_{\gamma^{-2 h}}^{\gamma^{-2 h+2}} d \alpha \int \frac{d k}{(2 \pi)^{2}} \\
& \times e^{-i k}{ }^{x}\left(i k_{0}+e(\vec{k})\right) e^{-\alpha p_{0}^{-2}\left[k_{0}^{2}+e(\vec{k})^{2}\right]} \chi\left(\omega \gamma^{-h} \vec{k}\right) .
\end{aligned}
$$

Here $\chi(t)=\pi^{-1 / 2} \int_{-\infty}^{t} d s \exp \left(-s^{2}\right)$ is a regularization of the step function.

In Appendix 1 we show (see also [BG], Appendix A) that, for any integer $m \geq 0$ :

$$
\left|\partial^{m} g_{\omega}^{(h)}(x)\right| \leq C_{m} \gamma^{h(1+m)} e^{-\kappa \gamma^{h}|x|}
$$

for some suitable constants $C_{m}$ and $\kappa$, independent of $h$. 
In the following we shall use also the definitions:

$$
\psi_{\omega, x}^{\sigma(\leq h)}=\sum_{k=-\infty}^{h} \psi_{\omega, x}^{\sigma(k)}, \quad \psi_{x}^{\sigma(\leq h)}=\sum_{\omega= \pm 1} e^{\imath \sigma p_{F} \omega \vec{x}} \psi_{\omega, x}^{\sigma(\leq h)}
$$

In order to evaluate $V_{\text {eff }}(\varphi)$, by (3.8) and (3.9), we should study the functional integral

$$
\int P\left(d \psi^{\leq 0}\right) e^{-\bar{V}^{(0)}\left(\psi^{\leq 0}+\varphi\right)}
$$

However, the analysis of this integral is more delicate in comparison to the analogous ultraviolet problem, because of the anomalous scaling. Therefore we split the problem into the simpler problem of defining the running couplings and into that of evaluating the effective potential. The first problem already emerges from the study of the integral (4.7) for $\varphi=0$, i.e. from the study of the normalization constant in (3.8); this analysis will be performed in this section and in the following one. The second problem will be faced up in Sect. 6 indirectly, through the analysis of the Schwinger functions, which are the physically relevant quantities.

Setting $\psi=\psi^{(\leq 0)}$ to simplify the notation, we represent the potential $\bar{V}^{(0)}(\psi)$, see (3.17), in terms of quasi-particle fields and we obtain:

$$
\begin{aligned}
\bar{V}^{(0)}(\psi)= & \lambda \int d x d y \sum_{\omega_{1} \omega_{4}} e^{i p_{F}\left[\left(\omega_{1}-\omega_{2}\right) \vec{x}+\left(\omega_{3}-\omega_{4}\right) \vec{y}\right]} \psi_{\omega_{1} x}^{+} \psi_{\omega_{2} x}^{-} v(x-y) \psi_{\omega_{3} y}^{+} \psi_{\omega_{4} y}^{-} \\
& +\nu \int d x \sum_{\omega_{1}, \omega_{2}} e^{i p_{F}\left(\omega_{1}-\omega_{2}\right) \vec{x}_{1}} \psi_{\omega_{1} x}^{+} \psi_{\omega_{2} x}^{-} \\
& +\alpha \int d x \sum_{\omega_{1}, \omega_{2}} e^{i p_{F}\left(\omega_{1}-\omega_{2}\right) \vec{x}} \psi_{\omega_{1} x}^{+} i \beta \omega_{2} \mathscr{D}_{\omega_{2}}^{-} \psi_{\omega_{2} x}^{-} \\
& +\sum_{n=1}^{\infty} \sum_{n_{1}, n_{2}} \sum_{\omega_{1}} \int d x_{1} \ldots d x_{2 n} e^{i p_{F}} \sum_{i=1}^{n}\left(\omega_{2} \vec{x}_{2}-\omega_{2}^{\prime} \vec{x}_{n+i}\right) \\
& \times \psi_{\omega_{1} x_{1}}^{+} \ldots \psi_{\omega_{n} x_{n}}^{+} \psi_{\omega_{1}^{\prime} x_{n+1}}^{-} \ldots \psi_{\omega_{n-n_{2}}^{\prime} x_{2 n-n_{2}}} \\
& \times i \omega_{n-n_{2}+1}^{\prime} \mathscr{D}_{\omega_{n-n_{2}+1}^{\prime}}^{-} \psi_{\omega_{n-n_{2}+1}^{\prime} x_{2 n-n_{2}+1}}^{\prime} \ldots i \omega_{n}^{\prime} \mathscr{D}_{\omega_{n}^{\prime}}^{-} \psi_{\omega_{n} x_{2 n}}^{-} \\
& \times \bar{W}_{n_{1} n_{2}}\left(z, x_{1} \ldots x_{2 n}\right)
\end{aligned}
$$

where $\beta \equiv p_{F} / m$, the covariant derivative $\mathscr{D}_{\omega}^{-}$is a differential operator acting only on the space coordinate, defined by:

$$
\mathscr{V}_{\omega}^{-}=\partial_{\vec{x}}+\frac{i \omega \partial_{\vec{x}}^{2}}{2 p_{F}}
$$

and the contribution of the third line in (3.17) has been included in the last term [see discussion related to (4.38) and (4.39) below]. 
The $\mathscr{D}_{\omega}^{-}$operator satisfies the following identity, which will play an important role in the following:

$$
\begin{aligned}
& \int d x \psi_{x}^{+(\leq h)} e\left(i \partial_{\vec{x}}\right) \psi_{x}^{-(\leq h)} \\
& =\sum_{\omega_{1}, \omega_{2}} \int d x e^{i p_{F}\left(\omega_{1}-\omega_{2}\right) \vec{x}} \psi_{\omega_{1} x}^{+(\leq h)} i \beta \omega_{2} \mathscr{D}_{\omega_{2}}^{-} \psi_{\omega_{2} x}^{-(\leq h)}
\end{aligned}
$$

It is now very natural to define the effective potential on scale $\gamma^{-h}$, for $h<0$, as in (3.25), through the expression:

$$
e^{-\bar{V}^{(h)}\left(\psi^{(\leq h)}\right)}=\frac{1}{\mathscr{N}} \int P\left(d \psi^{(h+1)}\right) \ldots \int P\left(d \psi^{(0)}\right) e^{-\bar{V}^{(0)}\left(\psi^{(\leq 0)}\right)} .
$$

We shall see in the following that this is not the correct definition, because of the anomalous scaling properties of the model. However we proceed for the moment with this definition in order to show where and why the problem arises.

As explained in [BG], we can isolate the relevant part of the effective potential by introducing a localization operator $\mathscr{L}$ which acts linearly on the monomials in the fields of the form $\prod_{i} \psi_{\omega_{i} x_{i}}^{\sigma_{2}}$ and is zero on all monomials of degree $\geq 6$. Its action on the monomials of degree 2 and 4 is generated by linearity from:

$$
\begin{aligned}
\mathscr{L}\left(\psi_{\omega_{1} x_{1}}^{+} \psi_{\omega_{2} x_{2}}^{+} \psi_{\omega_{3} x_{3}}^{-} \psi_{\omega_{4} x_{4}}^{-}\right)= & \frac{1}{2}\left[\psi_{\omega_{1} x_{1}}^{+} \psi_{\omega_{2} x_{1}}^{+} \psi_{\omega_{3} x_{1}}^{-} \psi_{\omega_{4} x_{1}}^{-}\right. \\
& \left.+\psi_{\omega_{1} x_{2}}^{+} \psi_{\omega_{2} x_{2}}^{+} \psi_{\omega_{3} x_{2}}^{-} \psi_{\omega_{4} x_{2}}^{-}\right], \\
\mathscr{L}\left(\psi_{\omega_{1} x_{1}}^{+} \psi_{\omega_{2} x_{2}}^{-}\right)= & \psi_{\omega_{1} x_{1}}^{+} \psi_{\omega_{2} x_{1}}^{-}+\left(x_{2}-x_{1}\right) \psi_{\omega_{1} x_{1}}^{+} \mathscr{D}_{\omega_{2}} \psi_{\omega_{2} x_{1}}^{-},
\end{aligned}
$$

where [see (4.9)]:

$$
\mathscr{D}_{\omega} \equiv\left(\partial_{t}, \mathscr{D}_{\omega}^{-}\right)
$$

We used in the second line of (4.12) the covariant derivative (4.9) instead of the normal space derivative, which could perhaps look more natural, for a reason which will be explained later [see remark following (4.30) below]; in any event our choice (4.12) differs from the other one only by an irrelevant term.

If $\mathscr{B}=1-\mathscr{L}$, we have also:

$$
\begin{aligned}
\mathscr{R}\left(\psi_{\omega_{1} x_{1}}^{+} \psi_{\omega_{2} x_{2}}^{+} \psi_{\omega_{3} x_{3}}^{-} \psi_{\omega_{4} x_{4}}^{-}\right) \\
=\frac{1}{2}\left[\psi_{\omega_{1} x_{1}}^{+} D_{21 \omega_{2}}^{+} \psi_{\omega_{3} x_{3}}^{-} \psi_{\omega_{4} x_{4}}^{-}+\psi_{\omega_{1} x_{1}}^{+} \psi_{\omega_{2} x_{1}}^{+} D_{31 \omega_{3}}^{-} \psi_{\omega_{4} x_{4}}^{-}\right. \\
\left.\quad+\psi_{\omega_{1} x_{1}}^{+} \psi_{\omega_{2} x_{1}}^{+} \psi_{\omega_{3} x_{1}}^{-} D_{41 \omega_{4}}^{-}\right] \\
\quad+\frac{1}{2}\left[D_{12 \omega_{2}}^{+} \psi_{\omega_{2} x_{2}}^{+} \psi_{\omega_{3} x_{3}}^{-} \psi_{\omega_{4} x_{4}}^{-}+\psi_{\omega_{1} x_{2}}^{+} \psi_{\omega_{2} x_{2}}^{+} D_{32 \omega_{3}}^{-} \psi_{\omega_{4} x_{4}}^{-}\right. \\
\left.\quad+\psi_{\omega_{1} x_{2}}^{+} \psi_{\omega_{2} x_{2}}^{+} \psi_{\omega_{3} x_{2}}^{-} D_{42 \omega_{4}}^{-}\right]
\end{aligned}
$$

where

$$
\begin{gathered}
D_{j i \omega_{j}}^{\sigma}=\psi_{\omega_{j} x_{\jmath}}^{\sigma}-\psi_{\omega_{j} x_{\imath}}^{\sigma}=\left(x_{j}-x_{i}\right) \int_{0}^{1} d r \partial \psi_{\omega_{j} x_{\jmath \imath}(r)}^{\sigma} \\
x_{j i}(r)=x_{i}+r\left(x_{j}-x_{i}\right), \quad \partial \equiv\left(\partial_{t}, \partial_{\vec{x}}\right)
\end{gathered}
$$


and for the quadratic term in the fields we have:

$$
\begin{aligned}
\mathscr{R}\left(\psi_{\omega_{1} x_{1}}^{+} \psi_{\omega_{2} x_{2}}^{-}\right)= & -\frac{i \omega_{2}}{2 p_{F}}\left(\vec{x}_{2}-\vec{x}_{1}\right) \psi_{\omega_{1} x_{1}}^{+} \partial_{\vec{x}}^{2} \psi_{\omega_{2} x_{1}}^{-} \\
& +\left(x_{2}-x_{1}\right)^{2} \psi_{\omega_{1} x_{1}}^{+} \int_{0}^{1} d r \int_{0}^{r} d s \partial^{2} \psi_{\omega_{2} x_{21}(s)}^{-}
\end{aligned}
$$

where

$$
\left(x_{2}-x_{1}\right)^{2} \int_{0}^{1} d r \int_{0}^{r} d s \partial^{2} \psi_{\omega_{2} x_{21}(s)}^{-}=\psi_{\omega_{2} x_{2}}^{-}-\psi_{\omega_{2} x_{1}}^{-}-\left(x_{2}-x_{1}\right) \partial \psi_{\omega_{2} x_{1}}^{-} .
$$

We plan to evaluate iteratively the integrals in the r.h.s. of (4.11), by rewriting at each step $\bar{V}^{(h)}$ in the form $\mathscr{L} \bar{V}^{(h)}+\mathscr{B} \bar{V}^{(h)}$. This implies that we have to consider the action of $\mathscr{L}$ also on other monomials of second and fourth order, besides those appearing in (4.8). We shall give now the complete list of the monomials that one has to take into account, for which the action of $\mathscr{L}$ does not give zero, together with the result of the application of $\mathscr{L}$ and $\mathscr{R}$, deduced from (4.12) by linearity.

In the case of the fourth order monomials there is only one more term on which $\mathscr{L}$ is not trivial, in principle; it is the one of the form $\psi_{x_{1} \omega_{1}}^{+} \partial \psi_{x_{2} \omega_{2}}^{+} \psi_{x_{3} \omega_{3}}^{-} \psi_{x_{4} \omega_{4}}^{-}$. This term can only appear if $x_{1}$ is an interpolated point, see (4.15), so that we really need the following equation:

$$
\begin{aligned}
& \mathscr{L} \psi_{x_{1} \vec{\omega}_{1}}^{+} D_{x_{2} x_{2^{\prime}} \vec{\omega}_{2}}^{+} \psi_{x_{3} \vec{\omega}_{3}}^{-} \psi_{x_{4} \vec{\omega}_{4}}^{-} \\
& =\frac{1}{2}\left[\psi_{x_{2} \vec{\omega}_{1}}^{+} \psi_{x_{2} \vec{\omega}_{2}}^{+} \psi_{x_{2} \vec{\omega}_{3}}^{-} \psi_{x_{2} \vec{\omega}_{4}}^{-}-\psi_{x_{2^{\prime}} \vec{\omega}_{1}}^{+} \psi_{x_{2^{\prime}} \vec{\omega}_{2}}^{+} \psi_{x_{2^{\prime}} \vec{\omega}_{3}}^{-} \psi_{x_{2^{\prime}} \vec{\omega}_{4}}^{-}\right] .
\end{aligned}
$$

By the anticommutation properties of the field, the r.h.s. can be different from zero only if $\omega_{1}=-\omega_{2}, \omega_{3}=-\omega_{4}$. However, in this case, the integration on the $x$ variables cancels it, because the monomial in the l.h.s. appears multiplied by a translation invariant function of the $x$-variables; furthermore the oscillating factor $e^{i p_{F}\left(\omega_{1} \vec{x}_{1}+\omega_{2} \vec{x}_{2}-\omega_{3} \vec{x}_{3}-\omega_{4} \vec{x}_{4}\right)}$ is also translation invariant, if $\omega_{1}=-\omega_{2}, \omega_{3}=-\omega_{4}$.

Hence, for our purpose:

$$
\mathscr{L} \psi_{x_{1} \omega_{1}}^{+} \partial \psi_{x_{2} \omega_{2}}^{+} \psi_{x_{3} \omega_{3}}^{-} \psi_{x_{4} \omega_{4}}^{-}=0
$$

and we do not have to consider any other localization operation on the fourth order monomials, besides that of (4.12).

In the case of the second order monomials, we have to consider the following localization operations:

$$
\begin{aligned}
\mathscr{L}\left(\psi_{\omega_{1} x_{1}}^{+} \mathscr{D}_{\omega_{2}} \psi_{\omega_{2} x_{2}}^{-}\right)= & \psi_{\omega_{1} x_{1}}^{+} \mathscr{D}_{\omega_{2}} \psi_{\omega_{2} x_{1}}^{-}, \\
\mathscr{C}\left(\psi_{\omega_{1} x_{1}}^{+} \partial \psi_{\omega_{2} x_{2^{\prime}}}^{-}\right)= & \psi_{\omega_{1} x_{1}}^{+} \mathscr{D}_{\omega_{2}} \psi_{\omega_{2} x_{1}}^{-}, \\
\mathscr{L}\left(\partial \psi_{\omega_{1} x_{1^{\prime}}}^{+} \psi_{\omega_{2} x_{2}}^{-}\right)= & \partial\left(\psi_{\omega_{1} x_{1^{\prime}}}^{+} \psi_{\omega_{2} x_{1^{\prime}}}^{-}\right)-\psi_{\omega_{1} x_{1^{\prime}}}^{+} \mathscr{D}_{\omega_{2}} \psi_{\omega_{2} x_{1^{\prime}}}^{-} \\
& +\left(x_{2}-x_{1^{\prime}}\right) \partial\left(\psi_{\omega_{1} x_{1^{\prime}}}^{+} \mathscr{D}_{\omega_{2}} \psi_{\omega_{2} x_{1^{\prime}}}^{-}\right) \\
\mathscr{L}\left(\partial \psi_{\omega_{1} x_{1^{\prime}}}^{+} \mathscr{D}_{\omega_{2}} \psi_{\omega_{2} x_{2}}^{-}\right)= & \partial\left(\psi_{\omega_{1} x_{1^{\prime}}}^{+} \mathscr{D}_{\omega_{2}} \psi_{\omega_{2} x_{1^{\prime}}}^{-}\right) \\
\mathscr{L}\left(\partial \psi_{\omega_{1} x_{1^{\prime}}} \partial \psi_{\omega_{2} x_{2^{\prime}}}^{-}\right)= & \partial\left(\psi_{\omega_{1} x_{1^{\prime}}}^{+} \mathscr{D}_{\omega_{2}} \psi_{\omega_{2} x_{1^{\prime}}}^{-}\right)
\end{aligned}
$$


and the corresponding $\mathscr{R}$ operations:

$$
\begin{aligned}
\mathscr{R}\left(\psi_{\omega_{1} x_{1}}^{+} \mathscr{D}_{\omega_{2}} \psi_{\omega_{2} x_{2}}^{-}\right)= & \left(x_{2}-x_{1}\right) \psi_{\omega_{1} x_{1}}^{+} \int_{0}^{1} d r \mathscr{D}_{\omega_{2}} \partial \psi_{\left.\omega_{2} x_{21(r)}\right)}^{-}, \\
\mathscr{R}\left(\psi_{\omega_{1} x_{1}}^{+} \partial \psi_{\omega_{2} x_{2^{\prime}}}^{-}\right)= & -\frac{i \omega_{2}}{2 p_{F}} \psi_{\omega_{1} x_{1}}^{+} \partial_{\vec{x}}^{2} \psi_{\omega_{2} x_{1}}^{-} \\
& +\left(x_{2^{\prime}}-x_{1}\right) \psi_{\omega_{1}}^{+}\left(x_{1}\right) \int_{0}^{1} d r \partial \partial \psi_{\omega_{2} x_{2^{\prime} 1(r)}}^{-}, \\
& -\frac{i \omega_{2}}{2 p_{F}} \psi_{\omega_{1} x_{1^{\prime}}}^{+} \partial_{\vec{x}}^{2} \psi_{\omega_{2} x_{1^{\prime}}}^{-} \\
& -\left(x_{2}-x_{1^{\prime}}\right) \partial \psi_{\omega_{1} x_{1^{\prime}}}^{+} \mathscr{D}_{\omega_{2}} \psi_{\omega_{2} x_{1^{\prime}}}^{-} \\
& -\left(x_{2}-x_{1^{\prime}}\right) \psi_{\omega_{1} x_{1^{\prime}}}^{+} \partial \mathscr{D}_{\omega_{2}} \psi_{\omega_{2} x_{1^{\prime}}}^{-} \\
\mathscr{R}\left(\partial \psi_{\omega_{1} x_{1^{\prime}}}^{+} \psi_{\omega_{2} x_{2}}^{-}\right)= & \left(x_{2}-x_{1^{\prime}}\right) \partial \psi_{\omega_{1} x_{1^{\prime}}}^{+} \int_{0}^{1} d r \partial \psi_{\omega_{2} x_{21^{\prime}(r)}}^{-} \\
\mathscr{R}\left(\partial \psi_{\omega_{1} x_{1^{\prime}}}^{+} \mathscr{D}_{\omega_{2}} \psi_{\omega_{2} x_{2}}^{-}\right)= & \partial \psi_{\omega_{1} x_{1^{\prime}}}^{+} \mathscr{D}_{\omega_{2}} \psi_{\omega_{2} x_{2}}^{-}-\partial\left(\psi_{\omega_{1} x_{1^{\prime}}}^{+} \mathscr{D}_{\omega_{2}} \psi_{\omega_{2} x_{1^{\prime}}}^{-}\right) \\
\mathscr{R}\left(\partial \psi_{\omega_{1} x_{1}}^{+} \partial \psi_{\omega_{2} x_{2}^{\prime}}^{-}\right)= & \partial \psi_{\omega_{1} x_{1^{\prime}}}^{+} \partial \psi_{\omega_{2} x_{2^{\prime}}}^{-}-\partial\left(\psi_{\omega_{1} x_{1^{\prime}}}^{+} \mathscr{D}_{\omega_{2}} \psi_{\omega_{2} x_{1^{\prime}}}^{-}\right)
\end{aligned}
$$

where the symbol $x_{j^{\prime}}$ is used to stress that $x_{j^{\prime}}$ is a point on the segment connecting $x_{j}$ and some other point.

In the r.h.s. of the last three of equations (4.20), some new local terms appear with respect to the second relation in the r.h.s. of (4.12). However, the field $\partial \psi_{\omega_{1} x_{1}}^{+}$in the 1.h.s. of (4.20) can appear only through a field $D_{12}^{+}$by interpolation, see (4.15). Hence one has really to consider the following localization operations:

$$
\begin{aligned}
\mathscr{L}\left(D_{12 \omega_{1}}^{+} \psi_{x_{3} \omega_{2}}^{-}\right)= & \psi_{x_{1} \omega_{1}}^{+} \psi_{x_{1} \omega_{2}}^{-}-\psi_{x_{2} \omega_{1}}^{+} \psi_{x_{2} \omega_{2}}^{-} \\
& +\left(x_{3}-x_{1}\right) \psi_{x_{1} \omega_{1}}^{+} \mathscr{D}_{\omega_{2}} \psi_{x_{1} \omega_{2}}^{-} \\
& -\left(x_{3}-x_{2}\right) \psi_{x_{2} \omega_{1}}^{+} \mathscr{D}_{\omega_{2}} \psi_{x_{2} \omega_{2}}^{-}, \\
\mathscr{L}\left(D_{12 \omega_{1}}^{+} \mathscr{D}_{\omega_{2}} \psi_{x_{3} \omega_{2}}^{-}\right)= & \psi_{x_{1} \omega_{1}}^{+} \mathscr{D}_{\omega_{2}} \psi_{x_{1} \omega_{2}}^{-}-\psi_{x_{2} \omega_{1}}^{+} \mathscr{D}_{\omega_{2}} \psi_{x_{2} \omega_{2}}^{-}, \\
\mathscr{L}\left(D_{12 \omega_{1}}^{+} D_{34 \omega_{2}}^{-}\right)= & \left(x_{3}-x_{4}\right)\left(\psi_{x_{1} \omega_{1}}^{+} \mathscr{D}_{\omega_{2}} \psi_{x_{1} \omega_{2}}^{-}-\psi_{x_{2} \omega_{1}}^{+} \mathscr{D}_{\omega_{2}} \psi_{x_{2} \omega_{2}}^{-}\right) .
\end{aligned}
$$

And we can conclude that, as a result of the localization operation on the effective potential, we get, for each scale, the following local monomials:

$$
\psi_{x+}^{+} \psi_{x-}^{-} \psi_{x+}^{-} \psi_{x-}^{-}, \quad \psi_{x \omega}^{+} \psi_{x \omega^{\prime}}^{-}, \quad \psi_{x \omega}^{+} i \omega^{\prime} \beta \mathscr{D}_{\omega^{\prime}}^{-} \psi_{x \omega^{\prime}}^{-}, \quad \psi_{x \omega}^{+} \partial_{t} \psi_{x \omega^{\prime}}^{-}
$$

multiplied by some constants, the running coupling constants of the model, that we shall indicate, respectively, with $\lambda_{h}, \gamma^{h} \nu_{h}, \alpha_{h}, \zeta_{h}$.

At first sight, the running coupling constants depend on the $\omega$ variables; however, we shall see that they are actually $\omega$-independent. 
The fourth order local part must have the form:

$$
\sum_{\omega} \int d x \lambda_{h}\left(\omega_{1}, \omega_{2}, \omega_{3}, \omega_{4}\right) e^{\imath p_{F}\left(\omega_{1}+\omega_{2}-\omega_{3}-\omega_{4}\right) \vec{x}} \psi_{\omega_{1} x}^{+(\leq h)} \psi_{\omega_{2} x}^{+(\leq h)} \psi_{\omega_{3} x}^{-(\leq h)} \psi_{\omega_{4} x}^{-(\leq h)}
$$

and recalling the anticommutation properties of fermions, we can write:

$$
\lambda_{h}\left(\omega_{1}, \omega_{2}, \omega_{3}, \omega_{4}\right)=-\frac{\lambda_{h}}{4} \omega_{1} \omega_{3} \delta_{\omega_{1},-\omega_{2}} \delta_{\omega_{3},-\omega_{4}}
$$

Hence we can rewrite the quartic relevant part in the simpler way:

$$
\lambda_{h} \int d x \psi_{+1 x}^{+(\leq h)} \psi_{-1 x}^{+(\leq h)} \psi_{-1 x}^{-(\leq h)} \psi_{+1 x}^{-(\leq h)}
$$

Let us now investigate the $\omega$-dependence of the running coupling constants associated with the quadratic terms in the effective potential on scale $\gamma^{-h}$. By the linearity of $\mathscr{L}$, we can calculate the local part in a different way. First we can do all the integrations in (4.11) without introducing the quasi-particle field representation; then we represent the effective potential in terms of the quasi-particle fields and finally we apply the localization operator. After the first step, the quadratic part of the effective potential on scale $\gamma^{-h}$, expressed in terms of particle fields, looks as follows:

$$
\begin{aligned}
\bar{V}^{[2](h)}= & \int d x d y v_{h}(x-y) \psi^{+}(x) \psi^{-}(y) \\
& +\int d x d y w_{h}(x-y) \psi^{+}(x) e\left(i \partial_{\vec{y}}\right) \psi^{-}(y)
\end{aligned}
$$

where $v_{h}$ and $w_{h}$ are rotation invariant kernels (this means, in one dimension, that they are even functions in the spatial coordinate); such property follows from the fact that the free propagator of the theory and the interaction are indeed rotation invariant.

We represent now $\bar{V}^{[2](h)}$ in terms of quasi-particle fields:

$$
\begin{aligned}
\bar{V}^{[2](h)}= & \sum_{\omega, \omega^{\prime}}\left[\int d x d y v_{h}(x-y) e^{\imath p_{F}\left(\omega \vec{x}-\omega^{\prime} \vec{y}\right)} \psi_{\omega x}^{+} \psi_{\omega^{\prime} y}^{-}\right. \\
& \left.+\int d x d y w_{h}(x-y) e^{i p_{F}\left(\omega \vec{x}-\omega^{\prime} \vec{y}\right)} \psi_{\omega x}^{+} i \beta \omega^{\prime} \mathscr{D}_{\omega^{\prime}}^{-} \psi_{\omega^{\prime} y}^{-}\right] .
\end{aligned}
$$

Hence the second order local part has the form:

$$
\begin{aligned}
\mathscr{S} \bar{V}^{[2](h)}= & \gamma^{h} \nu_{h} \sum_{\omega \omega^{\prime}} \int d x e^{i p_{F}\left(\omega-\omega^{\prime}\right) \vec{x}} \psi_{\omega x}^{+} \psi_{\omega^{\prime} x}^{-} \\
& +\alpha_{h} \sum_{\omega \omega^{\prime}} \int d x e^{i p_{F}\left(\omega-\omega^{\prime}\right) \vec{x}} \psi_{\omega x}^{+} i \beta \omega^{\prime} \mathscr{D}_{\omega^{\prime}}^{-} \psi_{\omega^{\prime} x}^{-} \\
& +\zeta_{h} \sum_{\omega \omega^{\prime}} \int d x e^{\imath p_{F}\left(\omega-\omega^{\prime}\right) \vec{x}} \psi_{\omega x}^{+} \partial_{t} \psi_{\omega^{\prime} x}^{-},
\end{aligned}
$$


where, if $\vec{z}$ is the spatial part of the two dimensional space-time vector $z$ and $z_{0}$ is its time component:

$$
\begin{aligned}
\gamma^{h} \nu_{h} & =\int d z v_{h}(z) e^{i p_{F} \omega^{\prime} \vec{z}} \\
\alpha_{h} & =\int d z e^{i p_{F} \omega^{\prime} \vec{z}}\left[w_{h}(z)+\frac{i}{\beta} \omega^{\prime} \vec{z} v_{h}(z)\right] \\
\zeta_{h} & =\int d z e^{\imath p_{F} \omega^{\prime} \vec{z}}\left(-z_{0}\right) v_{h}(z) .
\end{aligned}
$$

The latter definitions immediately imply that $\nu_{h}, \alpha_{h}$, and $\zeta_{h}$ are independent of the $\omega$ 's, as a consequence of the rotation invariance of the theory.

The previous observation has another consequence, which will play an important role in the following analysis. The structure of (4.22) is, in fact, not suitable for the dimensional bounds that we want to discuss: the r.h.s. of (4.22) is written as a sum of terms which do not vanish when $x_{1}=x_{2}$, i.e. we loose track of the fact that the 1.h.s. of (4.22) vanishes for $x_{1}=x_{2}$, a property which is manifest in the l.h.s. through the field $D_{12 \omega_{1}}^{+}$; this is disappointing because the property of vanishing of the 1.h.s. must be used to regularize the vertex where the field $D_{12 \omega_{1}}^{+}$appeared at a previous scale, along the iterative construction of $\bar{V}^{(h)}$.

As a consequence we cannot have good bounds for the contributions to $\nu_{h}, \alpha_{h}, \zeta_{h}$, coming from the individual terms in the r.h.s. of (4.22). However, if $\omega_{1}=\omega_{2}$, it is easy to see that the contributions arising from the second and the third of (4.22) cancel out, because of the translation invariance of the theory, by an argument similar to that used in the remark following (4.18) and leading to the "effective validity" of (4.19) (see also [BG], Sect. 11). In the first of (4.22) the translation invariance implies that, if $\omega_{1}=\omega_{2}=\omega$, the r.h.s. can be replaced by $\left(x_{1}-x_{2}\right) \psi_{x_{1} \omega}^{+} \mathscr{D}_{\omega} \psi_{x_{1} \omega}^{-}$, and in this way the needed $\left(x_{1}-x_{2}\right)$ factor is explicitly exhibited.

To summarize, if $\omega_{1}=\omega_{2}=\omega$, we can replace (4.22) with:

$$
\begin{aligned}
\mathscr{L}\left(D_{12 \omega}^{+} \psi_{x_{3} \omega}^{-}\right) & =\left(x_{1}-x_{2}\right) \psi_{x_{1} \omega}^{+} \mathscr{D}_{\omega} \psi_{x_{1} \omega}^{-}, \\
\mathscr{L}\left(D_{12 \omega}^{+} \mathscr{D} \psi_{x_{3} \omega}^{-}\right) & =\mathscr{L}\left(D_{12 \omega}^{+} D_{34 \omega}^{-}\right)=0 .
\end{aligned}
$$

The previous properties are not valid anymore, if $\omega_{1} \neq \omega_{2}$; hence there would be a serious problem, if we had to bound the contributions to the effective potential associated with the local terms in the r.h.s. of (4.22) for all $\omega_{1}, \omega_{2}$. But this is not the case, since we know a priori that $\nu_{h}, \alpha_{h}, \zeta_{h}$ are independent of $\omega_{1}, \omega_{2}$ and we are not interested in the single contributions building the running coupling constants expansions, but only in their sums. Hence we can choose to compute the running coupling constants via their expansions valid for $\omega_{1}=\omega_{2}$, which does not give any trouble, as we shall see.

Before starting the inductive evaluation of (4.11), we write:

$$
\bar{V}^{(0)}\left(\psi^{\leq(0)}\right)=\mathscr{L} \bar{V}^{(0)}\left(\psi^{\leq(0)}\right)+\mathscr{R} \bar{V}^{(0)}\left(\psi^{\leq(0)}\right)
$$


It is easy to see that:

$$
\begin{aligned}
\mathscr{L} \bar{V}^{(0)}\left(\psi^{\leq(0)}\right)= & \lambda_{0} \int d x \psi_{+1 x}^{+(\leq 0)} \psi_{-1 x}^{+(\leq 0)} \psi_{-1 x}^{-(\leq 0)} \psi_{+1 x}^{-(\leq 0)} \\
& +\nu_{0} \int d x \sum_{\omega_{1} \omega_{2}} e^{i p_{F}\left(\omega_{1}-\omega_{2}\right) \vec{x}} \psi_{\omega_{1} x}^{+(\leq 0)} \psi_{\omega_{2} x}^{-(\leq 0)} \\
& +\alpha_{0} \int d x \sum_{\omega_{1} \omega_{2}} e^{\imath p_{F}\left(\omega_{1}-\omega_{2}\right) \vec{x}} \psi_{\omega_{1} x}^{+(\leq 0)} i \beta \mathscr{D}_{\omega_{2}}^{-} \psi_{\omega_{2} x}^{-(\leq 0)} \\
& +\zeta_{0} \int d x \sum_{\omega_{1} \omega_{2}} e^{i p_{F}\left(\omega_{1}-\omega_{2}\right) \vec{x}} \psi_{\omega_{1} x}^{+(\leq 0)} \partial_{t} \psi_{\omega_{2} x}^{-(\leq 0)}
\end{aligned}
$$

for suitably chosen $\lambda_{0}, \nu_{0}, \alpha_{0}, \zeta_{0}$, and:

$$
\mathscr{R} \bar{V}^{(0)}\left(\psi^{\leq(0)}\right)=\sum_{n=1}^{\infty} \sum_{\varrho \in I_{n}} \int d \underline{x} \bar{W}_{\varrho}(z, \underline{x}) M_{\varrho}\left(\psi^{\leq(0)}\right) .
$$

Here $I_{n}$ is the finite set of different monomials of the form:

$$
M_{\varrho}(\psi)=\left(\prod_{\imath=1}^{n} \Phi_{\imath}^{+}\right)\left(\prod_{j=1}^{n} \Phi_{j}^{-}\right)
$$

where $\Phi_{i}^{+}$has to be chosen between the fields [see (4.21)]:

$$
e^{\imath p_{F} \omega \vec{x}} \psi_{\omega x}^{+}, \quad e^{\imath p_{F} \omega \vec{x}_{2}} \int_{0}^{1} d r \partial \psi_{\omega x_{21}(r)}^{+}
$$

and $\Phi_{\jmath}^{-}$has to be chosen between the fields:

$$
\begin{aligned}
& e^{-i p_{F} \omega \vec{x}} \psi_{\omega x}^{-}, \quad e^{-\imath p_{F} \omega \vec{x}} \mathscr{D}_{\omega} \psi_{\omega x}^{-}, \\
& \left(-\frac{i \omega}{2 p_{F}}\right) e^{-i p_{F} \omega \vec{x}_{2}} \partial_{\vec{x}}^{2} \psi_{\omega x_{1}}^{-}, \quad e^{-\imath p_{F} \omega \vec{x}_{2}} \int_{0}^{1} d r \partial \psi_{\omega x_{21}(r)}^{-}, \\
& e^{-i p_{F} \omega \vec{x}_{2}} \int_{0}^{1} d r \mathscr{D}_{\omega} \partial \psi_{\omega x_{21}(r)}^{-}, \quad e^{-i p_{F} \omega \vec{x}_{2}} \int_{0}^{1} d r \int_{0}^{r} d s \partial^{2} \psi_{\omega x_{21}(s)}^{-} .
\end{aligned}
$$

Moreover, in (4.34) $\underline{x}$ represents the set of points appearing as labels of the fields in the monomial $M_{\varrho}$.

Remark. The running couplings $\lambda_{0}, \nu_{0}, \alpha_{0}$, and $\zeta_{0}$ are in fact convergent series of the bare couplings $z=(\lambda, \nu, \alpha)$, uniformly in the u.v. cutoff $N$. This follows from (3.18) for the contributions coming from $\bar{W}_{n_{1} n_{2}}$, with $n_{1}+n_{2}=2$ or 4 , but there is, at first sight, a problem for the contributions to $\nu_{0}, a_{0}$, and $\zeta_{0}$, coming from $\tilde{W}_{2}$. However we can use here (3.20), which implies, for example, that the contribution of $\tilde{W}_{2}$ to $\alpha_{0}$ is:

$$
-2 m \int d x e^{i p_{F} \vec{x}} \tilde{W}_{2}(x)=2 m \int d x\left[1-e^{\imath p_{F} \vec{x}}\right] \tilde{W}_{2}(x),
$$


which can be bounded by:

$$
2 m p_{F} \int d x|\vec{x}|\left|\tilde{W}_{2}(x)\right|,
$$

a finite bound uniformly in $N$ by (3.19).

It is also important to stress that, by (3.18) and (3.19), the kernels of (4.34) are convergent series of $z$, which satisfy for $|z|$ small enough the bound:

$$
\int d \underline{x}\left|\bar{W}_{\varrho}(z, \underline{x})\right| e^{\frac{\kappa}{2} d^{(0)}(\underline{x})} \leq|\Lambda|(C|z|)^{\max \{1, n-1\}},
$$

and the power on the r.h.s. can be really 1 only in the case of the term coming from the action of $\mathscr{R}$ on the first term of (4.8).

The first order in the bare constants gives:

$$
\begin{aligned}
\lambda_{0} & =2 \lambda \int d \vec{x} \bar{v}(\vec{x})\left[1-\cos \left(2 p_{F} \vec{x}\right)\right], \\
\nu_{0} & =\nu+2 \lambda \int d \vec{x} \bar{v}(\vec{x})\left[e^{i p_{F} \vec{x}} R(0, \vec{x})-R(0)\right], \\
\alpha_{0} & =\alpha+2 \lambda \frac{i}{\beta} \int d \vec{x} \bar{v}(\vec{x}) R(0, \vec{x}) \vec{x} e^{\imath p_{F} \vec{x}}, \quad \zeta_{0}=0 .
\end{aligned}
$$

We can now start the inductive evaluation of (4.11), by applying at each step the localization operator to the effective potential. We will obtain for $\mathscr{L} V^{(h)}$ a formula like (4.33), with $\lambda_{0}, \nu_{0}, \alpha_{0}, \zeta_{0}$ replaced by $\left(\lambda_{h}, \gamma^{h} \nu_{h}, \alpha_{h}, \zeta_{h}\right)$; and $\left(\lambda_{h}, \nu_{h}, \alpha_{h}, \zeta_{h}\right) \equiv r_{h}$ will be called the running coupling constants of frequency $h$. The $r_{h}$ can be expressed as a series of the running coupling constants of frequencies $k \geq h+1$, i.e. $r_{h+1} \ldots r_{0}$. We could show that this series, called the beta functional, is convergent if all the running coupling constants $r_{h+1}, \ldots, r_{0}$ stay bounded within a certain radius of convergence, and we could show as well that the irrelevant part of the effective potential can be written as a convergent series of $r_{h+1}, \ldots, r_{0}$ (for a general discussion on the beta-functional see for example [G]).

Of course, in order to use this result, we would also have to prove that the running constants really do stay bounded, at least if the bare constants are small enough. However, if we try to pursue this program, we immediately find a difficulty. In fact, if we calculate the beta functional at second order, we find ([BG, $G])$ :

$$
\begin{aligned}
\lambda_{h-1} & =\lambda_{h}, \\
\alpha_{h-1} & =\alpha_{h}+\beta_{2} \lambda_{h}^{2}+O\left(\gamma^{h}\right), \\
\zeta_{h-1} & =\zeta_{h}+\beta_{2} \lambda_{h}^{2}+O\left(\gamma^{h}\right)
\end{aligned}
$$

with $\beta_{2} \neq 0$.

The latter equations imply that, at the second order, $\lambda_{h}$ neither does increase nor does decrease; so we need the third order to decide what happens to $\lambda_{h}$. However, even if we suppose that the third order for $\lambda_{h}$, once calculated, will imply that $\lambda_{h}$ goes to zero when $h \rightarrow-\infty$, the best that we can hope to find for its behaviour is clearly a rate $\sqrt{1 /|h|}$. Looking at second order equations for $\alpha_{h}$ and $\zeta_{h}$, this implies that $\alpha_{h}$ and $\zeta_{h}$ go to infinity at least as $\sum_{h} 1 /|h|$, i.e. we get out of the established domain of convergence of the beta functional in a finite number of steps. 
From the mathematical point of view this is a big trouble, because it makes it impossible to construct a perturbation theory for the model; from the physical point of view this means, as it is well known, that the expectation of the number of particles with fixed momentum, in the one dimensional Fermi gas, has a singularity, at the Fermi momenta $\pm p_{F}$, of a different kind with respect to the free case, where it is simply discontinuous.

Hence we need to introduce a different type of scaling, allowing us to study the nature of the singularity on the Fermi surface via a consistent perturbation theory.

\section{The Effective Potential in the Infrared Region. Running Couplings and Anomalous Scaling. The Ground State Energy}

A new and more general scaling approach is based on a representation of the field $\psi^{(\leq 0)}$ alternative to the one described by (4.2)-(4.4).

In fact there are many ways to represent the Grassmannian integration $P\left(d \psi^{(\leq 0)}\right)$ with $\psi^{(\leq 0)}=\sum_{h=-\infty}^{0} \psi^{(h)}$, each parametrized by an arbitrary sequence $Z_{0}=$ $1, Z_{-1}, Z_{-2}, \ldots$ of non-zero numbers.

Denote $P_{Z_{h}}(d \psi)$ the Grassmannian integration with propagator $\frac{1}{Z_{h}} g^{(\leq h)}$ and $\tilde{P}_{Z_{h}}(d \psi)$ the integration with propagator $\frac{1}{Z_{h}} \tilde{g}^{(h)}$, where $\tilde{g}^{(0)}=g^{(0)}$ and $\tilde{g}^{(h)}$ will be fixed below.

The $\tilde{g}^{(-1)}$ will be fixed, given the sequence $Z_{h}$, starting from the following obvious identities:

$$
\begin{aligned}
P_{Z_{0}}\left(d \psi^{(\leq 0)}\right)= & \tilde{P}_{Z_{0}}\left(d \psi^{(0)}\right) P_{Z_{0}}(d \psi \leq(-1)) \\
= & \tilde{P}_{Z_{0}}\left(d \psi^{(0)}\right)\left[P_{Z_{0}}\left(d \psi^{\leq(-1)}\right) e^{-\left(Z_{-1}-Z_{0}\right)\left(\psi^{(\leq-1)+}, T \psi^{(\leq-1)-}\right)-t_{-1}^{\prime}|\Lambda|}\right] \\
& \times e^{+\left(Z_{-1}-Z_{0}\right)\left(\psi^{(\leq-1)+}, T \psi^{(\leq-1)-}\right)+t_{-1}^{\prime}|\Lambda|}
\end{aligned}
$$

where $T$ is the differential operator $\partial_{t}+e\left(i \partial_{\vec{x}}\right)$ and $t_{-1}^{\prime}$ is a normalization constant such that the term in square brackets is a normalized Grassmannian integration with propagator:

$$
\left[Z_{0}\left(g^{(\leq-1)}\right)^{-1}+\left(Z_{-1}-Z_{0}\right) T\right]^{-1},
$$

and, according to Sect. 4 :

$$
g^{(\leq h)}(k)=\frac{C_{h}(k)^{-1}}{-i k_{0}+e(\vec{k})}, \quad C_{h}(k)=e^{\gamma^{-2 h}\left(k_{0}^{2}+e(\vec{k})^{2}\right) p_{0}^{-2}}=e^{\gamma^{-2 h} \beta(k)},
$$

with $\beta(k)$ being defined here. Therefore the normalization constant is:

$$
t_{-1}^{\prime}=\int \frac{d^{2} k}{(2 \pi)^{2}} \log \left(1+\frac{Z_{-1}-Z_{0}}{Z_{0}} e^{-\gamma^{2}\left(k_{0}^{2}+e(\vec{k})^{2}\right) p_{0}^{-2}}\right)
$$

and, finally, from (5.2) we define $\tilde{g}^{(-1)}$ as:

$$
\frac{\left[Z_{0} C_{-1}(k)+z Z_{0}\right]^{-1}}{-i k_{0}+e\left(k_{1}\right)}=\frac{\left[Z_{-1} C_{-2}(k)\right]^{-1}}{-i k_{0}+e\left(k_{1}\right)}+\frac{1}{Z_{-1}} \tilde{g}^{(-1)}(k)
$$


where, if $z=\left(Z_{-1}-Z_{0}\right) / Z_{0}$ :

$$
\begin{gathered}
\tilde{g}^{(-1)}(k)=g^{(-1)}(k)+r^{(-1)}(k), \quad g^{(-1)}(k)=\frac{e^{-\gamma^{2} \beta(k)}-e^{-\gamma^{4} \beta(k)}}{-i k_{0}+e\left(k_{1}\right)}, \\
r^{(-1)}(k)=\frac{e^{-\gamma^{2} \beta(k)}\left(1-e^{-\gamma^{2} \beta(k)}\right)}{-i k_{0}+e\left(k_{1}\right)} \frac{z}{1+z e^{-\gamma^{2} \beta(k)}} .
\end{gathered}
$$

Hence (5.1) becomes:

$$
\begin{aligned}
P\left(d \psi^{(\leq 0)}\right)= & \tilde{P}_{Z_{0}}\left(d \psi^{(0)}\right) P_{Z_{0}}\left(d \psi^{(\leq-1)}\right) \\
= & \tilde{P}_{Z_{0}}\left(d \psi^{(0)}\right) \tilde{P}_{Z_{-1}}\left(d \psi^{(-1)}\right) P_{Z_{-1}}\left(d \psi^{(\leq-2)}\right) \\
& \times e^{\left(Z_{-1}-Z_{0}\right)\left(\psi^{(\leq-1)}, T \psi^{(\leq-1)}\right)+t_{-1}^{\prime}|\Lambda|}
\end{aligned}
$$

By iteration we define $z_{h}=\left(Z_{h}-Z_{h+1}\right) / Z_{h+1}$ and $\tilde{g}^{(h)}$ as:

$$
\frac{\left[Z_{h+1} C_{h}(k)+z_{h} Z_{h+1}\right]^{-1}}{-i k_{0}+e\left(k_{1}\right)}=\frac{\left[Z_{h} C_{h-1}(k)\right]^{-1}}{-i k_{0}+e\left(k_{1}\right)}+\frac{1}{Z_{h}} \tilde{g}^{(h)}(k),
$$

so that we must take:

$$
\begin{aligned}
& \tilde{g}^{(h)}(k)=g^{(h)}(k)+r^{(h)}(k), \\
& r^{(h)}(k)=\frac{e^{-\gamma^{-2 h} \beta(k)}\left(1-e^{-\gamma^{-2 h} \beta(k)}\right)}{-i k_{0}+e\left(k_{1}\right)} \frac{z_{h}}{1+z_{h} e^{-\gamma^{-2 h} \beta(k)}},
\end{aligned}
$$

arriving at the representation, valid for all $k \leq-1$ :

$$
\begin{aligned}
P_{Z_{0}}\left(d \psi^{(\leq 0)}\right)= & \left(\prod_{h=-\infty}^{0} \tilde{P}_{Z_{h}}\left(d \psi^{(h)}\right)\right) \\
& \times\left(\prod_{h=-\infty}^{-1} e^{\left(Z_{h}-Z_{h+1}\right)\left(\psi^{(\leq h)}, T \psi^{(\leq h)}\right)+t_{h}^{\prime}|\Lambda|}\right) \\
= & \left(\prod_{h=k+1}^{0} \tilde{P}_{Z_{h}}\left(d \psi^{(h)}\right)\right) \\
& \times\left(\prod_{h=k+1}^{-1} e^{\left(Z_{h}-Z_{h+1}\right)\left(\psi^{(\leq h)}, T \psi^{(\leq h)}\right)+t_{h}^{\prime}|\Lambda|}\right) P_{Z_{k+1}}\left(d \psi^{(\leq k)}\right)
\end{aligned}
$$

with $\psi^{(\leq p)}=\sum_{h=-\infty}^{p} \psi^{(h)}$. 
We recover the decomposition of Sect. 4 by setting $Z_{h} \equiv 1$.

The freedom in the choice of the sequence $Z_{h}$ can be used to cancel terms proportional to $\left(\psi^{(\leq h)}, T \psi^{(\leq h)}\right)$ arising in the calculation of the effective potential.

We define the anomalous effective potentials $V^{(h)}$ via:

$$
\begin{aligned}
& e^{-V^{(h)}\left(\sqrt{Z_{h}} \psi^{(\leq h)}\right)}=\int \prod_{h^{\prime}=h+1}^{0} \tilde{P}_{Z_{h^{\prime}}}\left(d \psi^{\left(h^{\prime}\right)}\right) \\
& \quad \times e^{-V^{(0)}\left(\sqrt{Z_{0}} \psi^{(\leq 0)}\right)+\sum_{h^{\prime}=h}^{-1}\left[\left(Z_{h^{\prime}}-Z_{h^{\prime}+1}\right)\left(\psi^{\left(\leq h^{\prime}\right)}, T \psi^{\left(\leq h^{\prime}\right)}\right)+t_{h^{\prime}}^{\prime}|\Lambda|\right]},
\end{aligned}
$$

where $V^{(0)}\left(\psi^{(\leq 0)}\right) \equiv \bar{V}^{(0)}\left(\psi^{(\leq 0)}\right)$; so that:

$$
\begin{aligned}
\int & P\left(d \psi^{(\leq 0)}\right) e^{V^{(0)}\left(\psi^{(\leq 0)}\right)} \\
& =\int P_{Z_{h+1}}\left(d \psi^{(\leq h)}\right) e^{-\left(Z_{h}-Z_{h+1}\right)\left(\psi^{(\leq h)}, T \psi^{(\leq h)}\right)-t_{h}^{\prime}|\Lambda|} e^{-V^{(h)}\left(\sqrt{Z_{h}} \psi^{(\leq h)}\right)} \\
& =\int \tilde{P}_{Z_{h}}\left(d \psi^{(h)}\right) P_{Z_{h}}\left(d \psi^{(<h)}\right) e^{-V^{(h)}\left(\sqrt{Z_{h}} \psi^{(\leq h)}\right)} .
\end{aligned}
$$

In the following the sequence $Z_{h}$ can and will be chosen so that $\mathscr{L} V^{(h)}$ contains no term proportional to $\int\left(\psi^{(\leq h)}, \partial_{t} \psi^{(\leq h)}\right)$. And we shall apply the above ideas to study the ground state energy per unit volume. To understand in detail the mechanism behind the recursive definition of $Z_{h}$ we perform in detail the analysis of $Z_{-1}$.

Let us consider the first step of our construction, i.e. the integration of the field of frequency $h=0$. If we put $Z_{0}=1$, we can write the partition function

$$
\begin{aligned}
e^{E|\Lambda|} & =\int P_{Z_{0}}\left(d \psi^{(\leq-1)}\right) P_{Z_{0}}\left(d \psi^{(0)}\right) e^{-\bar{V}^{(0)}\left[\sqrt{Z_{0}}\left(\psi^{(0)}+\psi^{(\leq-1)}\right)\right]} \\
& =\int P_{Z_{0}}\left(d \psi^{(\leq-1)}\right) e^{-\bar{V}^{(-1)}\left(\sqrt{Z_{0}} \psi^{(\leq-1)}\right)}
\end{aligned}
$$

where the Grassmannian integration can be thought of in terms of quasi-particle fields as well as of particle fields.

We note that, if $Z_{0}$ had been different from $1, \bar{V}^{(-1)}$ would have changed only because the external lines (of the generic graph contributing to it) would represent $\sqrt{Z_{0}} \psi_{x}^{(\leq-1)}$ instead of $\psi_{x}^{(\leq-1)}$; in fact, in the internal lines, the factor $1 / Z_{0}$ coming from the propagator is compensated by the factor $\left(\sqrt{Z_{0}}\right)^{2}$ coming from the fields which are contracted in that line.

We now split $\bar{V}^{(-1)}$, as a functional of the quasi-particle field, into its quadratic relevant part plus the rest. Such splitting is a quasi-particle invariant one in the sense that the two parts of the splitting of $\bar{V}^{(-1)}$ can be expressed independently in terms of particle fields.

Hence we write:

$$
\begin{aligned}
& \bar{V}^{(-1)}\left(\sqrt{Z_{0}} \psi^{(\leq-1)}\right)=Z_{0} \sum_{\omega \omega^{\prime}} \int d x e^{i p_{F}\left(\omega-\omega^{\prime}\right)} \vec{x}\left[n \psi_{\omega x}^{+(\leq-1)} \psi_{\omega^{\prime} x}^{-(\leq-1)}\right. \\
& \left.\quad+a \psi_{\omega x}^{+(\leq-1)} i \beta \omega^{\prime} \mathscr{D}_{\omega^{\prime}}^{-} \psi_{\omega^{\prime} x}^{-(\leq-1)}+z \psi_{\omega x}^{+(\leq-1)} \partial_{t} \psi_{\omega^{\prime} x}^{-(\leq-1)}\right]+t_{-1}|\Lambda| \\
& \quad+\mathscr{R}\left[\text { quadratic part of } \bar{V}^{(-1)}\right] \\
& \left.\quad+(\mathscr{L}+\mathscr{R}) \text { [higher than quadratic part of } \bar{V}^{(-1)}\right] .
\end{aligned}
$$


The constant part $t_{-1}|\Lambda|$ of $\bar{V}^{(-1)}$ is introduced here explicitly as we wish to compute the ground state energy density $E$. The constants $n, a$, and $z$ are (of course, as $Z_{0}=1$ ) the old running coupling constants of frequency $h=-1$, i.e. $\nu_{-1}, \alpha_{-1}$, and $\zeta_{-1}$. In terms of particle fields, (5.14) can be written:

$$
\begin{aligned}
& \bar{V}^{(-1)}\left(\sqrt{Z_{0}} \psi^{(\leq-1)}\right)=Z_{0} \int d x\left[n \psi_{x}^{+(\leq-1)} \psi_{x}^{-(\leq-1)}\right. \\
& \left.\quad+a \psi_{x}^{+(\leq-1)} e\left(i \partial_{\vec{x}}\right) \psi_{x}^{-(\leq-1)}+z \psi_{x}^{+(\leq-1)} \partial_{t} \psi_{x}^{-(\leq-1)}+t_{-1}|\Lambda|\right] \\
& \quad+[\text { all the other terms expressed in terms of particle fields] }
\end{aligned}
$$

Let us remark that, by (5.15), (5.11):

$$
\begin{aligned}
\int & P_{Z_{0}}\left(d \psi^{(0)}\right) P_{Z_{0}}\left(d \psi^{(\leq-1)}\right) e^{-V^{(0)}\left(\sqrt{Z_{0}} \psi^{(\leq 0)}\right)} \\
& =\int P_{Z_{0}}\left(d \psi^{(\leq-1)}\right) e^{-\bar{V}^{(-1)}\left(\sqrt{Z_{0}} \psi^{(\leq-1)}\right)} \\
& =\frac{1}{\mathscr{N}} \int d \psi^{(\leq-1)} e^{-Z_{0}\left(\psi^{(\leq-1)},\left(C_{-1}+z\right) T \psi^{(\leq-1)}\right)} e^{-V^{(-1)}\left(\sqrt{Z_{-1}} \psi^{(\leq-1)}\right)} \\
& =\int P_{Z_{-1}}\left(d \psi^{(\leq-2)}\right) \tilde{P}_{Z_{-1}}\left(d \psi^{(-1)}\right) e^{-V^{(-1)}\left(\sqrt{Z_{-1}} \psi^{(\leq-1)}\right)}
\end{aligned}
$$

where $\mathscr{N}$ is a (formal) normalization factor, and provided $Z_{-1}, V^{(-1)}$ are defined appropriately, and precisely as:

$$
\begin{aligned}
Z_{-1}= & Z_{0}(1+z) \\
V^{(-1)}\left(\sqrt{Z_{-1}} \psi^{(\leq-1)}\right)= & \int d x\left[Z_{0}(a-z) \psi_{x}^{+} e\left(i \partial_{\vec{x}}\right) \psi_{x}^{-}+Z_{0} n \psi_{x}^{+} \psi_{x}^{-}\right] \\
& +\left(t_{-1}+t_{-1}^{\prime}\right)|\Lambda| \\
& +[\text { all the other terms as in }(5.15)] .
\end{aligned}
$$

The above identities define $Z_{-1}$ and the anomalous effective potential $V^{(-1)}$ in terms of particle fields. Here we see that the property that the effective potential can be expressed in terms of particle fields holds; this is a "symmetry property," of great importance in the following, and we shall refer to it by saying that our definition of anomalous scaling preserves the quasi-particle invariance of the theory.

We can write r.h.s. of (5.16) as:

$$
\begin{aligned}
& \int P_{Z_{-1}}\left(d \psi^{(\leq-2)}\right) \tilde{P}_{Z_{-1}}\left(d \psi^{(-1)}\right) e^{-V^{(-1)}\left(\sqrt{Z_{-1}}\left[\psi^{(\leq-2)}+\psi^{(-1)}\right]\right)} \\
& =\int P_{Z_{-1}}\left(d \psi^{(\leq-2)}\right) e^{-\tilde{V}^{(-2)}\left(\sqrt{Z_{-1}} \psi^{(\leq-2)}\right)}
\end{aligned}
$$

and the r.h.s. has the same structure of the 1.h.s. in (5.13), (5.16) so that we are in a position to repeat the procedure.

Remark If we rewrite the anomalous effective potential $V^{(-1)}$ (which, from now on, will be called simply the "effective potential," dropping the adjective anomalous) in 
terms of quasi-particle fields and we call $V^{(-1)[2 n]}$ the part of $V^{(-1)}$ which contains the monomials of order $2 n$, we get:

$$
\begin{aligned}
V^{(-1)}(\psi)= & \sum_{\omega \omega^{\prime}} \int d x e^{i p_{F}\left(\omega-\omega^{\prime}\right) \vec{x}} \\
& \times\left[\frac{Z_{0}}{Z_{-1}}(a-z) \psi_{\omega x}^{+} i \beta \omega^{\prime} \mathscr{D}_{\omega^{\prime}}^{-} \psi_{\omega^{\prime} x}^{-}+\frac{Z_{0}}{Z_{-1}} n \psi_{\omega x}^{+} \psi_{\omega^{\prime} x}^{-}\right] \\
& +\int d x\left(\frac{Z_{0}}{Z_{-1}}\right)^{2} l \psi_{x+}^{+} \psi_{x-}^{+} \psi_{x+}^{-} \psi_{x-}^{-} \\
& +\mathscr{R} V^{(-1)[2]}\left(\sqrt{\frac{Z_{0}}{Z_{-1}} \psi}\right)+\mathscr{B} V^{(-1)[4]}\left(\sqrt{\frac{Z_{0}}{Z_{-1}} \psi}\right) \\
& +\sum_{n>2} V^{(-1)[2 n]}\left(\sqrt{\frac{Z_{0}}{Z_{-1}}} \psi\right)+\left(t_{-1}+t_{-1}^{\prime}\right)|\Lambda|,
\end{aligned}
$$

where the constant $l$ in front of the quartic relevant term is of course the old $\lambda_{-1}$ (because $Z_{0}=1$ ). Hence we have only four relevant terms, including the vacuum terms $\left(t_{-1}+t_{-1}^{\prime}\right)$ in $V^{(-1)}(\psi)$, and therefore only four running coupling constants which, by (5.19), are given by the equations:

$$
\begin{array}{cc}
\gamma^{-2} \vartheta_{-1}=\left(t_{-1}+t_{-1}^{\prime}\right), & \gamma^{-1} \nu_{-1}=\frac{Z_{0}}{Z_{-1}} n, \\
\delta_{-1}=\frac{Z_{0}}{Z_{-1}}(a-z), & \lambda_{-1}=\frac{Z_{0}^{2}}{Z_{-1}^{2}} l
\end{array}
$$

with $z, n, l, a$ and $t_{-1}, t_{-1}^{\prime}$ convergent series of the bare constants.

We repeat step by step for all single scale integrations the procedure followed in going from (5.13) to (5.18). We define:

$$
e^{-\tilde{V}^{(h)}\left(\sqrt{Z_{h+1}} \psi^{(\leq h)}\right)}=\int \tilde{P}_{Z_{h+1}}\left(d \psi^{(h+1)}\right) e^{-V^{(h+1)}\left[\sqrt{Z_{h+1}}\left(\psi^{(h+1)}+\psi^{(\leq h)}\right)\right]}
$$

where $\psi^{(h)}$ is the field of propagator $\tilde{g}^{(h)} / Z_{h}$ defined above.

And we write also the analogous of Eqs. (5.14) and (5.15) (we shall call $n_{h}, a_{h}$, $z_{h}, l_{h}$ the coefficients of the local terms) and we define the (anomalous) effective potential of frequency $h$, with running coupling constants $\lambda_{h}, \nu_{h}, \delta_{h}$, as in (5.16), that is:

$$
\begin{aligned}
& \int P_{Z_{h+1}}\left(d \psi^{(\leq h)}\right) e^{-\tilde{V}^{(h)}\left(\sqrt{Z_{h+1}} \psi^{(\leq h)}\right)} \\
&=\int \tilde{P}_{Z_{h}}\left(d \psi^{(h)}\right) P_{Z_{h}}\left(d \psi^{(<h)}\right) e^{-V^{(h)}\left(\sqrt{Z_{h}} \psi^{(\leq h)}\right)},
\end{aligned}
$$

where, as in (5.17) and (5.20), we have [setting the $z$ introduced in (5.17) $z \equiv z_{-1}$ ]:

$$
Z_{h}=Z_{h+1}\left(1+z_{h}\right)
$$


and

$$
\gamma^{2 h} \vartheta_{h}=t_{h}+t_{h}^{\prime}, \quad \gamma^{h} \nu_{h}=\frac{Z_{h+1}}{Z_{h}} n_{h}, \quad \delta_{h}=\frac{Z_{h+1}}{Z_{h}}\left(a_{h}-z_{h}\right), \quad \lambda_{h}=\frac{Z_{h+1}^{2}}{Z_{h}^{2}} l_{h}
$$

with

$$
t_{h}^{\prime}=\int \frac{d^{2} k}{(2 \pi)^{2}} \log \left(1+\frac{Z_{h}-Z_{h+1}}{Z_{h+1}} e^{-\gamma^{-2 h}\left(k_{0}^{2}+e(\vec{k})^{2}\right) p_{0}^{-2}}\right)
$$

The calculation of the integral in the r.h.s. of (5.21) is done by using the quasiparticle representation of the fields; hence we define, as in (4.2):

$$
\psi_{x}^{\sigma(h)} \equiv \sum_{\omega= \pm 1} e^{i \sigma p_{F} \omega \vec{x}} \psi_{\omega x}^{\sigma(h)}
$$

In Appendix 1 we show that the field $\psi_{\omega x}^{(h)}$ has a propagator $\tilde{g}_{\omega}^{(h)}(x) / Z_{h}$ satisfying, for any integer $m \geq 0$, the bound:

$$
\left|\partial^{m} \tilde{g}_{\omega}^{(h)}(x)\right| \leq C_{m} \gamma^{h(1+m)} e^{-\kappa \gamma^{h}|x|}
$$

for some suitable constants $C_{m}$ and $\kappa$, independent of $h$, if $\left|z_{h}\right|$ is sufficiently small, for example:

$$
\left|z_{h}\right| \leq \frac{1}{2}
$$

In other words, the fields $\psi_{\omega x}^{(h)}$ are, really, on scale $\gamma^{-h}$.

We can describe the structure of $V^{h}$ in terms of a tree expansion, similar to that used for the u.v. problem. By (5.21), we can write:

$$
\begin{aligned}
\tilde{V}^{(h)} & \left(\sqrt{Z_{h+1}} \psi^{(\leq h)}\right) \\
& =\sum_{n=1}^{\infty} \frac{1}{n !} E_{h+1}^{T}\left[V^{(h+1)}\left(\sqrt{Z_{h+1}} \psi^{(\leq h+1)}\right), \ldots, V^{(h+1)}\left(\sqrt{Z_{h+1}} \psi^{(\leq h+1)}\right)\right],
\end{aligned}
$$

where we used the symbol $E_{h}^{T}$ to denote the truncated expectation with respect to the field of covariance $\tilde{g}^{(h)} / Z_{h}$.

We can obtain $V^{(h)}$ from $\tilde{V}^{(h)}$ with the following obvious modification of the $\mathscr{L}$ operation:

$$
V^{(h)}(\psi)=\mathscr{L}^{*} \tilde{V}^{(h)}\left(\sqrt{\frac{Z_{h+1}}{Z_{h}}} \psi\right)+\mathscr{B} \tilde{V}^{(h)}\left(\sqrt{\frac{Z_{h+1}}{Z_{h}}} \psi\right),
$$

where $\mathscr{L}^{*} \tilde{V}^{(h)}$ differs from $\mathscr{L} \tilde{V}^{(h)}$ only because it does not contain anymore the addend proportional to $\psi^{+} \partial_{t} \psi^{-}$; moreover the coefficients of the other four relevant terms are written as in the left- hand sides of Eqs. (5.24).

The tree expansion of $V^{(k)}$ is produced by iteration of (5.29) and (5.30), starting from (4.32)-(4.34), as in the u.v. case. However we need to change the definition of the trees, which was devised in Sect. 3 in connection with Eq. (3.28); in fact the latter is no longer valid, mainly because of the localization procedure. But we shall still call $\mathscr{T}_{n}$ the family of labeled trees with $n$ endpoints (see Sect. 3, Fig. 2) and we shall use the definitions of Sect. 3, when there is no difference of meaning in the notation; the differences are stressed in the following items. 
Fig. 8.

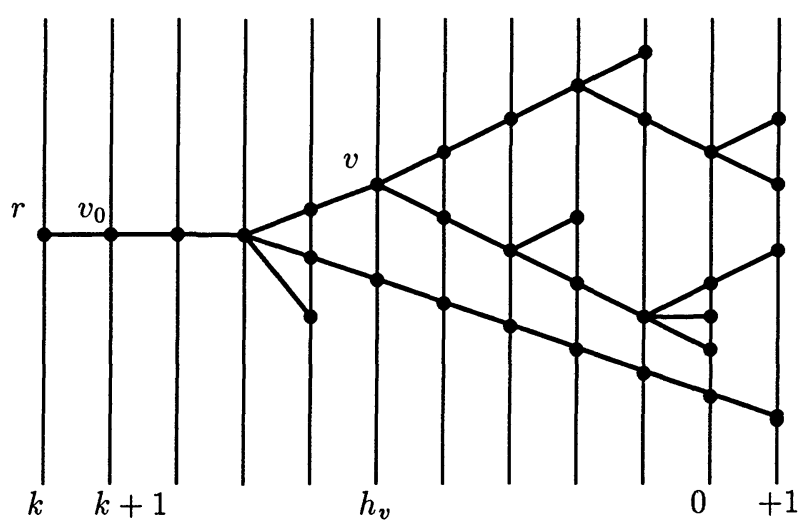

1) The frequency index of the vertical lines (see Fig. 2 in Sect. 3 and compare it with Fig. 8) takes all the integer values between $k$ and +1 , analogously to what happens in the u.v. case, but the endpoints do not necessarily belong to the line with index +1 . Moreover also the intersections of the vertical lines with the endbranches have to be considered as trivial vertices.

2) To each endpoint we associate either one of the four different local relevant terms, and in such a case we shall say that the endpoint is of type $\nu, \delta, \lambda, \vartheta$ (the latter, however, can be associated only with the trivial tree) respectively, or one of the irrelevant terms of (4.34), and we shall call the endpoint of type $M_{\varrho}$, see (4.35). Note that there is an infinite number of choices for $M_{\varrho}$, because after the u.v. integration the potential will contain monomials of any order in the field; the monomials $M_{\varrho}$ will thus be called monomials generated by the u.v. integration

If the endpoint is of type $\nu, \delta$, or $\lambda$ and $n \geq 2$, there is no trivial vertex between the endpoint and the non-trivial vertex $v$ immediately preceding it on the tree; this implies that the endpoint belongs to the vertical line with frequency index $h_{v}+1$. Moreover, $h_{v}$ is equal to the frequency index of the running coupling constant associated with the endpoint.

If the endpoint is of type $\nu, \delta, \lambda$ or $\vartheta$ and $n=1$, there is only the vertex $v_{0}$ on the tree, besides the endpoint, and the frequency index of the corresponding running coupling constant is equal to $h_{v_{0}}=h_{k+1}$. +1 .

If the endpoint is of type $M_{\varrho}$, it must belong to the vertical line of frequency index

3) As in Sect. 3, we associate with each tree a set $\underline{x}$ of space vertices and a set of field variables (called simply fields in the following), that is all the integration variables and all the field variables appearing in the terms associated with the endpoints. We shall still attach a label $f=1, \ldots, n_{\tau}$ to each field to distinguish them. Analogously we define $\underline{x}_{v}$ for the subtree starting from the vertex $v$. Furthermore, as in the u.v. case, the tree selects a class of graphs with lines of different frequencies and such graphs can be collected into families; such families are characterized by the choice of the external lines in all the subgraphs related to the tree vertices.

There is however an important difference, which is a consequence of the localization procedure; we shall have to describe the effect of the $\mathscr{R}$ operator on all trivial or non-trivial vertices of the tree $\tau$, and we have to specify if the tree contributes to the relevant part of the effective potential or to the irrelevant one. 
If the tree contributes to the irrelevant part, we shall associate a label $r_{v}$ with each trivial or non-trivial vertex. The label $r_{v}$ depends on the set of external lines in the subgraph related to the vertex; it specifies one of the monomials which are produced by the $\mathscr{R}$ operation at the corresponding frequency [see (4.21)] and note that there is only one choice when the subgraph associated to the vertex has more than four external lines).

If the tree contributes to the relevant local part, we associate a label $r_{v}$ in the same way to all vertices, except $v_{0}$, which will carry a label $L=L_{0}, L_{1}, L_{2}, L_{3}$, if the graph contributes to the running coupling constant $\vartheta_{k}, \lambda_{k}, \nu_{k}, \delta_{k}$, respectively.

We stress that the total number of choices for all the labels $\left\{r_{v}\right\}$ is of order $C^{m}$, if $m$ is the number of non-trivial vertices [as one sees from (4.21)].

4) The Grassmannian field variables can be those appearing in the lists (4.36), (4.37) or similar ones (see also next item); it will be important the remark that at most two derivatives can act on each single field. We shall use a label $u$ to distinguish the different possibilities. The field variables depend also on the label $\omega$, but in the following we shall not indicate such dependence, as it will not play any role in the rest of this section.

5) A Grassmannian field variable can depend upon more than one space vertex $x$, if it is involved in one or more $\mathscr{R}$ operations associated with the tree (of course as long as it remains an external field with respect to the graph associated to the tree vertices); in particular this can happen at an endpoint, if it belongs to a term of type $M_{\varrho}$, see (4.34). Furthermore, the label $u$ changes each time an $\mathscr{B}$ operation acts in a non-trivial way on the field, together with the set of space points on which it depends, see (4.14), (4.16), (4.21). Such sets of points are uniquely determined, once the set of indices $\left\{r_{v}\right\}$ is fixed. In all cases the sets contain a special point, namely the space vertex from which the field emerged before the first application of the $\mathscr{R}$ operation, or the localization point, if the field is associated with an external line of the total graph and the tree contributes to the local part; the special point also appears in the factor $e^{i p_{F} \sigma \omega \vec{x}}$ included in the definition of the field variables (4.36) and (4.37). In agreement with the above remarks we shall use for the fields the notation:

$$
\Psi_{x \underline{x}_{v}^{\prime}}^{u_{v}}
$$

where $u_{v}$ is the label in the vertex $v$ and $\underline{x}_{v}^{\prime} \subset \underline{x}_{v}$ is the set of space vertices from which the field depends, besides $x$ (and the set $\underline{x}_{v}^{\prime}$ can be empty).

We can now proceed in a way very similar to the ultraviolet case, with some natural changes due to the anomalous scaling procedure and to the different propagaors involed. We write

$$
V^{(k)}\left(\psi^{(\leq k)}\right)=\sum_{n=1}^{\infty} \sum_{\tau \in \mathscr{T}_{n}} V^{(k)}\left(\tau, \psi^{(\leq k)}\right)
$$

and

$$
V^{(k)}\left(\tau, Z_{k}^{1 / 2} \psi^{(\leq k)}\right)=\int d \underline{x}_{v_{0}} \sum_{P_{v_{0}}}\left(Z_{k}\right)^{\frac{1}{2}\left|P_{v_{0}}\right|} \tilde{\psi}^{(\leq k)}\left(P_{v_{0}}\right) V^{(k)}\left(\tau, P_{v_{0}}, \underline{x}_{v_{0}}\right)
$$

where, in the integral over the space-time coordinates, also the sum over the $\omega$ 's is included and the dependence of $V^{(k)}$ on the $\omega$ 's is not explicitly indicated; furthermore 
$P_{v_{0}}$ is a subset of $I_{v_{0}}=\left\{1, \ldots, n_{\tau}\right\}$ and

$$
\tilde{\psi}^{(\leq k)}\left(P_{v_{0}}\right)=\prod_{f \in P_{v_{0}}} \Psi_{x(f)\left(\underline{x}_{v_{0}}^{\prime}\right)(f)}^{\left(u_{f}\right) v_{0}(\leq k)}
$$

Note that the 1.h.s. of (5.34) is not completely identified by the set $P_{v_{0}}$, but we shall use it all of the same for sake of simplicity.

If $P_{v_{0}}$ is empty, i.e. if we consider a vacuum contribution, the corresponding contribution to $V^{(k)}$ will be written as a constant times $|\Lambda|$.

In order to simplify further the notation, we shall suppose, from now on, that there is no endpoint of type $M_{\varrho}$. It will be clear that the following analysis extends to the general case without any difficulty. This is equivalent to setting at the beginning the u.v. cut-off at $h=0$.

If $n=1$ (i.e. if the tree has only one line) and the endpoint is of type $\vartheta, \lambda, \nu$ or $\delta$, there is only one contribution to the sums in the r.h.s. of (5.33) and

$$
V^{(k)}\left(\tau, P_{v_{0}}, x\right)=\gamma^{2 k} \vartheta_{k}, \lambda_{k}, \gamma^{k} \nu_{k}, \delta_{k}
$$

respectively.

If $n \geq 2$ and $\tau^{1} \ldots \tau^{s v_{0}}$ are the subtrees starting from $v_{0}$ (the first vertex above the root) the definitions are such that

$$
\left.V^{(k)}\left(\tau, Z_{k}^{1 / 2} \psi^{(\leq k)}\right)=\bigodot \frac{1}{s_{v_{0}} !} E_{k+1}^{T}\left[V^{(k+1)}\left(\tau^{1}, Z_{k+1}^{1 / 2} \psi^{(\leq k+1)}\right), \ldots\right)\right],
$$

where the symbols $E_{h}, E_{h}^{T}$ denote the expectations with respect to a measure with covariance $Z_{h}^{-1} \tilde{g}^{(h)}$ (we reserved the symbols $\mathscr{E}_{h}, \mathscr{E}_{h}^{T}$ for the expectations with respect to the measure with covariance $\tilde{g}^{(h)}$ ) and $\left(\mathcal{O}\right.$ is equal to $\mathscr{L}^{*}$, if the tree contributes to the local part of the potential, or $\mathscr{R}$, if it contributes to the non-local irrelevant part.

The relations (5.33) and (5.36) imply that

$$
\begin{aligned}
& V^{(k)}\left(\tau, Z_{k}^{1 / 2} \psi^{(\leq k)}\right)=\int d \underline{x}_{v_{0}} \sum_{P_{v_{0}^{1}}, P_{v_{0}}} \prod_{v_{0}}^{s_{v_{0}}} V^{(k+1)}\left(\tau^{i}, P_{v_{0}^{i}}, \underline{x}_{v_{0}^{i}}\right) \\
& \quad \times\left(Z_{k+1}\right)^{\frac{1}{2} \sum_{i}\left|P_{v_{0}^{i}}\right|} \odot \frac{1}{s_{v_{0}} !} E_{k+1}^{T}\left[\tilde{\psi}^{(\leq k+1)}\left(P_{v_{0}^{1}}\right), \ldots, \tilde{\psi}^{(\leq k+1)}\left(P_{v_{0}} s_{v_{0}}\right)\right] .
\end{aligned}
$$

We write now, as in the u.v. case,

$$
\tilde{\psi}^{(\leq k+1)}\left(P_{v_{0}^{i}}\right)=\sum_{Q_{v_{0}^{2}}} \tilde{\psi}^{(<k+1)}\left(Q_{v_{0}^{2}}\right) \tilde{\psi}^{(k+1)}\left(P_{v_{0}^{2}} \backslash Q_{v_{0}^{i}}\right)
$$

By using the relations

$$
E_{h}^{T}\left[\tilde{\psi}^{h}\left(P_{1}\right), \ldots, \tilde{\psi}^{h}\left(P_{s}\right)\right]=\left(Z_{h}\right)^{-\frac{1}{2} \sum_{i}\left|P_{\imath}\right|} \mathscr{E}_{h}^{T}\left[\tilde{\psi}^{h}\left(P_{1}\right), \ldots, \tilde{\psi}^{h}\left(P_{s}\right)\right]
$$


(5.37) can be rewritten as

$$
\begin{aligned}
& V^{(k)}\left(\tau, Z_{k}^{1 / 2} \psi^{(\leq k)}\right)=\int d \underline{x}_{v_{0}} \sum_{P_{v_{0}^{1}}, \quad, P_{v_{0}} s_{0}} \sum_{Q_{v_{0}^{1}}, \quad, Q} \prod_{v_{0}} \prod_{i=1}^{s_{v_{0}}} V^{(k+1)}\left(\tau^{\imath}, P_{v_{0}^{2}}, \underline{x}_{v_{0}^{2}}\right) \\
& \times\left(Z_{k+1}\right)^{\frac{1}{2}\left|P_{v_{0}}\right|} \odot\left[\tilde{\psi}^{(\leq k)}\left(P_{v_{0}}\right)\right] \\
& \times \frac{1}{s_{v_{0}} !} \mathscr{E}_{k+1}^{T}\left[\tilde{\psi}^{(k+1)}\left(P_{v_{0}^{1}} \backslash Q_{v_{0}^{1}}\right), \ldots, \tilde{\psi}^{(k+1)}\left(P_{v_{0}} s_{v_{0}} \backslash Q_{v_{0}} s_{v_{0}}\right)\right]
\end{aligned}
$$

where $Q_{v_{0}^{i}} \subset P_{v_{0}^{i}}$ and $P_{v_{0}}=\bigcup_{i} Q_{v_{0}^{i}}$ represents the external lines of the subgraph associated with $v_{0}$.

The situation is now slightly different if compared with the u.v. case, because the propagator associated with $E_{h}$ contains the factor $Z_{h}^{-1}$.

We can rewrite $(5.40)$ as

$$
\begin{aligned}
& V^{(k)}\left(\tau, Z_{k}^{1 / 2} \psi^{(\leq k)}\right)=\int d \underline{x}_{v_{0}} \sum_{P_{v_{0}}}\left(Z_{k+1}\right)^{\frac{1}{2}\left|P_{v_{0}}\right|} \tilde{\psi}^{(\leq k)}\left(P_{v_{0}}\right) \\
& \quad \times \sum_{P_{v_{0}^{1}}, P_{v_{0} s_{v_{0}}}}\left(\xi_{v_{0}}-\xi_{v_{0}}^{\prime}\right)^{\bar{z}_{v_{0}}} \prod_{i=1}^{s_{v_{0}}} V^{(k+1)}\left(\tau^{\imath}, P_{v_{0}^{i}}, \underline{x}_{v_{0}^{i}}\right) \\
& \quad \times \frac{1}{s_{v_{0}} !} \mathscr{E}_{k+1}^{T}\left[\tilde{\psi}^{(k+1)}\left(P_{v_{0}^{1}} \backslash Q_{v_{0}^{1}}\right), \ldots, \tilde{\psi}^{(k+1)}\left(P_{v_{0}} s_{v_{0}} \backslash Q_{v_{0} s_{v_{0}}}\right)\right]
\end{aligned}
$$

where $\left(\xi_{v_{0}}-\xi_{v_{0}}^{\prime}\right)^{\bar{z}_{v_{0}}}$ is the factor, depending on the space vertices, which is produced by the $\mathscr{R}$ operation and is selected by the label $r_{v_{0}} ; \bar{z}_{v_{0}}$ is a positive integer less or equal to 2 and $\left(\xi_{v_{0}}-\xi_{v_{0}}^{\prime}\right)^{\bar{z}_{0}}$ must be interpreted, if $\bar{z}_{v_{0}}=2$, as a tensor of rank two.

Note that $\tilde{\psi}^{(\leq k)}\left(P_{v_{0}}\right)$ has in general a different meaning in (5.40) and in (5.41), as a consequence of the 9 operation [see also the remark after (5.34)].

From (5.41) we get a recurrence relation for $V^{(k)}\left(\tau, P_{v_{0}}, \underline{x}_{v_{0}}\right)$,

$$
\begin{aligned}
& V^{(k)}\left(\tau, P_{v_{0}}, \underline{x}_{v_{0}}\right)=\left(\frac{Z_{k+1}}{Z_{k}}\right)^{\frac{1}{2}\left|P_{v_{0}}\right|}\left(\xi_{v_{0}}-\xi_{v_{0}}^{\prime}\right)^{\bar{z}_{v_{0}}} \\
& \quad \times \sum_{P_{v_{0}^{1}}, P_{v_{0}} \sum_{v_{0}}} \prod_{i=1}^{s_{v_{0}}} V^{(k+1)}\left(\tau^{\imath}, P_{v_{0}^{i}}, \underline{x}_{v_{0}^{i}}\right) \\
& \quad \times \frac{1}{s_{v_{0}} !} \mathscr{E}_{k+1}^{T}\left[\tilde{\psi}^{(k+1)}\left(P_{v_{0}^{1}} \backslash Q_{v_{0}^{1}}\right), \ldots, \tilde{\psi}^{(k+1)}\left(P_{v_{0}} s_{v_{0}} \backslash Q_{v_{0}} s_{v_{0}}\right)\right]
\end{aligned}
$$


By iterating (5.42) and using (5.35), we can write the following closed expression:

$$
\begin{aligned}
V^{(k)}\left(\tau, P_{v_{0}}, \underline{x}_{v_{0}}\right)= & \sum_{\left\{P_{v}\right\}} \prod_{v \text { not e p }}\left(\frac{Z_{h_{v}}}{Z_{h_{v}-1}}\right)^{\frac{1}{2}\left|P_{v}\right|}\left(\xi_{v}-\xi_{v}^{\prime}\right)^{\bar{z}_{v}} \\
& \times \frac{1}{s_{v} !} \mathscr{E}_{h_{v}}^{T}\left[\tilde{\psi}^{\left(h_{v}\right)}\left(P_{v^{1}} \backslash Q_{v^{1}}\right), \ldots, \tilde{\psi}^{\left(h_{v}\right)}\left(P_{v^{s_{v}}} \backslash Q_{v^{s_{v}}}\right)\right] \\
& \times \prod_{i \in S_{\lambda}} \lambda_{h_{\imath}} \prod_{i \in S_{\nu}} \gamma^{h_{i}} \nu_{h_{\imath}} \prod_{i \in S_{\delta}} \delta_{h_{\imath}},
\end{aligned}
$$

where $S_{\alpha}$ denotes the set of endpoints of type $\alpha$ (recall that we are supposing there is no endpoint of type $M_{\varrho}$ ) and $h_{i}$ is the frequency of the non-trivial vertex which precedes the endpoint $i$.

The symbol $\sum_{\left\{P_{v}\right\}}$ denotes the sum over all the compatible choices of the subsets $P_{v}$ in all the non-trivial vertices of the tree, except $v_{0}$; such subsets are constrained by the same inclusion relations of the ultraviolet case. Hence the following constraints must hold:

$$
Q_{v} \subset P_{v}, \quad P_{v}=\bigcup Q_{v_{i}}
$$

As in the u.v. case, we now define the kernels

$$
W^{(k)}\left(\tau, P_{v_{0}}, \underline{x}^{\left(P_{v_{0}}\right)}=\int d\left(\underline{x} \backslash \underline{x}^{\left(P_{v_{0}}\right)} V^{(k)}\left(\tau, P_{v_{0}}, \underline{x}\right)\right.\right.
$$

so that

$$
\begin{aligned}
& V^{(k)}\left(\tau, Z_{k}^{1 / 2} \psi^{(\leq k)}\right)=\sum_{P_{v_{0}}} \int d \underline{x}^{\left(P_{v_{0}}\right.} W^{(k)}\left(\tau, P_{v_{0}}, \underline{x}^{\left(P_{v_{0}}\right)}\right) \\
& Z_{k}^{\frac{1}{2}\left|P_{v_{0}}\right|} \tilde{\psi}^{(\leq k)}\left(P_{v_{0}}\right) .
\end{aligned}
$$

Here $\underline{x}^{\left(P_{v_{0}}\right)}$ is the set of points on which the monomial $\tilde{\psi}^{(\leq k)}\left(P_{v_{0}}\right)$ depends (recall that there can be more than one point for each field). In particular $\underline{x}^{\left(P_{v_{0}}\right)}$ is a single point (or an empty set), if the tree contributes to a local (or vacuum) term, and in that case $W^{(k)}$ is a constant, (by translation invariance), whose value is used to calculate the running coupling constants of frequency $k$.

Let us now suppose that we know all the constants $\lambda_{h}, \nu_{h}, \delta_{h}, Z_{h}, \vartheta_{h}$, with $h>k$. In order to get from (5.46) the values of the kernels, we must first calculate $Z_{k}$. It is easy to see, by using (5.23) and (5.29), that we can write

$$
\frac{Z_{k}}{Z_{k+1}}=1+z_{k}=1+\sum_{n=2}^{\infty} \sum_{\tau \in \mathscr{T} n} \tilde{W}_{2, t}^{(k)}(\tau),
$$

where $\tilde{W}_{2, t}^{(k)}(\tau)$ is obtained by applying the $\mathscr{L}$ operator to the monomials with two external lines associated with the tree and then summing the coefficients of $\psi_{\omega x}^{+(\leq k)} \partial_{t} \psi_{\omega x}^{-(\leq k)}$, divided by $Z_{k+1}$. 
We can now calculate the new coupling constants and we get, $\forall k \leq-1$,

$$
\begin{aligned}
\lambda_{k} & =\left(\frac{Z_{k+1}}{Z_{k}}\right)^{2}\left[\lambda_{k+1}+\sum_{n=2}^{\infty} \sum_{\tau \in \mathscr{T}_{n}, r_{v_{0}}=L_{1}} W_{4}^{(k)}(\tau)\right], \\
\delta_{k} & =\left(\frac{Z_{k+1}}{Z_{k}}\right)\left[\delta_{k+1}+\sum_{n=2}^{\infty} \sum_{\tau \in \widetilde{T}_{n}, r_{v_{0}}=L_{3}} W_{2, \delta}^{(k)}(\tau)\right], \\
\nu_{k} & =\left(\frac{Z_{k+1}}{Z_{k}}\right)\left[\gamma \nu_{k+1}+\gamma^{-k} \sum_{n=2}^{\infty} \sum_{\tau \in \mathscr{T}_{n}, r_{v_{0}}=L_{2}} W_{2, \nu}^{(k)}(\tau)\right], \\
\vartheta_{k} & =\left[\gamma^{2} \vartheta_{k+1}+\gamma^{-2 k} \sum_{n=2}^{\infty} \sum_{\tau \in \mathscr{T}_{n}, r_{v_{0}}=L_{0}} W_{0}^{(k)}(\tau)\right],
\end{aligned}
$$

where the constants $W_{\alpha}^{(k)}$ are defined in an obvious way through (5.45), taking into account the remark about the independence of $\omega$ of the r.c.c., allowing us to restrict to consider only the terms with two external lines having the same $\omega$ label. Furthermore $\lambda_{0}, \nu_{0}$ and $\delta_{0}=\alpha_{0}$ are defined as in Sect. 4 [see (4.41) for their first order values in the bare constants].

Let us define

$$
r_{h}=\left(\lambda_{h}, \delta_{h}, \nu_{h}\right), \quad \varepsilon_{k}=\max _{h \geq k}\left|r_{h}\right|
$$

where the $r_{h}$ can take also complex values; then we can formulate the main result of this section:

Theorem 2. There exists a constant $\bar{\varepsilon}>0$, such that, if

$$
\varepsilon_{k+1} \leq \bar{\varepsilon}
$$

and, for some $c_{2}>0$,

$$
\sup _{k<h}\left|\frac{Z_{h}}{Z_{h-1}}\right| \leq e^{c_{2} \bar{\varepsilon}^{2}}
$$

then, for a suitable $\bar{\kappa}>0$,

$$
\int d \underline{x}^{\left(P v_{0}\right)} \sum_{\tau \in \mathbb{Z} n}\left|W^{(k)}\left(\tau, P_{v_{0}}, \underline{x}^{\left(P v_{0}\right)}\right)\right|\left|e^{\bar{\kappa} \gamma^{k} d\left(P_{v_{0}}\right)} \leq \gamma^{-k D\left(P_{v_{0}}\right)}\left(C \varepsilon_{k}\right)^{n}\right| \Lambda \mid
$$

where $d\left(P_{v_{0}}\right)$ is the length of the shortest tree graph connecting the set of points $\underline{x}^{\left(P_{v_{0}}\right)}$ and $D\left(P_{v_{0}}\right)$ is the "scaling dimension" of the monomial $\tilde{\psi}^{(\leq k)}\left(P_{v_{0}}\right)$, defined by

$$
D\left(P_{v_{0}}\right)=-2+\sum_{f \in P_{v_{0}}}\left(\frac{1}{2}+m_{f}\right),
$$

with $m_{f}$ being the order of the derivative operator applied to the field of label $f$.

Our proof will also imply that, see (5.47),

$$
\sum_{\tau}\left|\tilde{W}_{2, t}^{(k)}(\tau)\right| \leq\left(C \varepsilon_{k}\right)^{n} .
$$


Remarks. 1) It is easy to see that (5.52) and (5.54) imply that the series in the r.h.s. of (5.47) and (5.48) are convergent, uniformly in $k$, if (5.50) holds. Hence the condition (5.51) is satisfied for any $k$, for a suitable $c_{2}$, if $\bar{\varepsilon}$ is small enough and (5.50) stays valid. However it is not obvious at all that it is possible to choose $\bar{\varepsilon}$ so that the condition (5.50) is satisfied for all $k$. In order to get this result, one has to choose in a suitable way the constant $\nu$ of (2.31) and one has to compare the beta functional with that of the exactly soluble Luttinger model, as suggested in [BG, BGM], see Sect. 1. The problem will be discussed in detail in Sect. 7 below (and solved).

2) It is important to keep in mind that (5.45) and the bound (5.58) below allow us to get a version of the bound (5.52) without the integration, i.e. in the form

$$
\sum_{\tau \in \mathcal{T}_{n}}\left|W^{(k)}\left(\tau, P_{v_{0}}, \vec{x}^{\left(P_{v_{0}}\right)}\right)\right| \leq \sum_{h=k}^{0}\left(C \varepsilon_{h}\right)^{n} \gamma^{-h D\left(P_{v_{0}}\right)+2 h\left(\left|x^{\left(P_{v_{0}}\right)}\right|-1\right)} e^{-\bar{\kappa} \gamma^{h} d\left(P_{v_{0}}\right)},
$$

which is valid if the points belonging to $x^{\left(P_{v_{0}}\right)}$ are pairwise at a distance greater than $p_{0}^{-1}$, say [in order to avoid the trivial ultraviolet divergences due to the irrelevant terms present on scale 1, see (4.34)].

Hence the dimensionless potential, i.e. the kernels obtained from those of $V^{(k)}$ by multiplying them by $\gamma^{k D\left(P_{v_{0}}\right)-2 k\left(\mid x^{\left(P_{v_{0}} \mid-1\right)}\right.}$ and by replacing their $\vec{x}$ 's arguments by $\gamma^{-k} \vec{x}$ [we do not need to apply also a wave function renormalization, because the $Z_{h}$ factors were already extracted from the definition of the kernels, see (5.46)], verify

$$
\begin{gathered}
W_{\text {dimless }}^{(k)}\left(\vec{x}^{\left(P_{v_{0}}\right)}\right) \equiv \gamma^{k D\left(P_{v_{0}}\right)-2 k\left(\left|x^{\left(P v_{0}\right)}\right|-1\right)} \sum_{\tau} W^{(k)}\left(\tau, P_{v_{0}}, \gamma^{-k} x^{\left(P_{v_{0}}\right)}\right), \\
\left|W_{\text {dimless }}^{(k)}\left(\vec{x}^{\left(P v_{0}\right)}\right)\right|<e^{-\bar{\kappa} d\left(\vec{x}^{\left(P v_{0}\right)}\right)}\left(C \varepsilon_{k}\right)^{n}
\end{gathered}
$$

and the bound can be improved by replacing $\varepsilon_{k}$ with $\varepsilon_{k}^{\prime}=\sum_{h \geq k} \gamma^{-\vartheta(h-k)}\left|r_{h}\right|$ for some $\vartheta>0$ (so that if $r_{k} \underset{k \rightarrow-\infty}{\longrightarrow} 0$ the dimensionless potential tends to zero: a situation not arising in our problem but which can arise in asymptotically free theories).

3) The discussion of Sect. 7 will imply that the dimensionless potentials have a well defined limit as $k \rightarrow-\infty$, which can be interpreted as an exact fixed point of the renormalization group transformations that we consider, if regarded as a transformation of the dimensionless potentials. However the Schwinger functions are related to the non-rescaled potential, see (2.33). The latter also has a limit as $k \rightarrow \infty$, but this cannot be seen directly from the discussion in this section, because of the divergence of $Z_{h}$, which will be also proved in Sect. 7. Hence we cannot use (2.33) to study the Schwinger functions; in the next section we shall solve this problem by developing a more refined tree expansion, based on the application of the method of this section directly to the Schwinger functions. The structure of the effective potential on all scales found in this section will play an essential role, especially through the bound (5.68), in getting the "right" bounds on the asymptotic behaviour of the Schwinger functions.

It is important to remark that, also if the wave function renormalization constants were finite, we could not hope to use directly (2.33) to estimate the asymptotic behaviour of the Schwinger functions. We could only obtain a convergent expansion for their values at fixed distances.

4) The following general statement, ultimately relying on (5.52) and the latter improvement (5.68), can be also derived from the estimates of Sects. 5 and 6: the 
dimensionless effective potential $V_{\text {dimless }}^{(-\infty)}$ governs the corrections to the free asymptotic behaviour at large distances of the Schwinger functions (by "free" we mean here that the Schwinger functions can be evaluated from the pair Schwinger functions via the Wick rule, to leading order in the arguments distance), while the dimensional effective potential $V_{\text {eff }}\left[\right.$ formally equal to $\left.\lim _{h \rightarrow-\infty} V^{(h)}\left(\sqrt{Z_{h}} \cdot\right)\right]$ describes the correlations on all scales. Hence the vanishing of $V_{\text {dimless }}^{(-\infty)}$ has the physical meaning of trivial, i.e. free, asymptotic behaviour. The $V_{\text {dimless }}^{(-\infty)}$ is quite independent from the initial potential, it is universal; while $V_{\text {eff }}$ is, of course, explicitly dependent on the initial potential.

The proof of (5.52) is based on the following estimates of the truncated and simple expectations, which are very similar to those used in the u.v. case, and which are proved in Appendix 2:

$$
\begin{aligned}
\frac{1}{s !}\left|\mathscr{E}_{h}^{T}\left[\tilde{\psi}^{(h)}\left(P_{1}\right), \ldots, \tilde{\psi}^{(h)}\left(P_{s}\right)\right]\right| \leq & C^{\sum_{i}\left|P_{\imath}\right|} \gamma^{\frac{h}{2} \sum_{\imath} \sum_{j=0}^{2}(2 j+1)\left|P_{\imath}^{j}\right|} \\
& \times \frac{1}{s !} \sum_{T} \int d \underline{r}^{(P)} e^{-\kappa \gamma^{h} d_{T}^{*}\left(P_{1}, \quad, P_{s}\right)}
\end{aligned}
$$

where

1) $P^{3}$ denotes the subset of $P$ related to the fields containing a derivative operator of order $j$.

2) $\underline{r}^{(P)}$ is the set of interpolation parameters, appearing in the definition of some of the fields in $P$, see (4.36) and (4.37);

3) $T$ is an anchored tree graph between the clusters of space vertices (depending on $\left.\underline{r}^{(P)}\right)$ from which the fields labeled by $P_{1}, . ., P_{s}$ emerge; this means that $T$ is a set of lines connecting pairs of points in different clusters, and $T$ becomes a tree graph if one identifies all the points in the same cluster; $d_{T}^{*}\left(P_{1}, \ldots, P_{s}\right)$ is the sum of the lengths of the lines in $T$.

Hence, after some algebra, we can bound (5.43) as

$$
\begin{aligned}
\left|V^{(k)}\left(\tau, P_{v_{0}}, \underline{x}_{v_{0}}\right)\right| \leq & \sum_{\left\{P_{v}\right\}} \prod_{v \text { not e p }}\left|\frac{Z_{h_{v}}}{Z_{h_{v}-1}}\right|^{\frac{1}{2}\left|P_{v}\right|} C^{\sum_{i}\left|P_{v^{2}}\right|-\left|P_{v}\right|} \\
& \times J\left(\tau, P_{v_{0}}, \underline{x}_{v_{0}}\right) \cdot \gamma^{\frac{h_{v}}{2} \sum_{j=0}^{2}(2 j+1) \sum_{i}\left(\left|P_{v^{2}}^{j}\right|-\left|Q_{v^{2}}^{j}\right|\right)} \\
& \times\left(\prod_{i \in S_{\lambda}}\left|\lambda_{h_{i}}\right|\right)\left(\prod_{i \in S_{\nu}}\left|\gamma^{h_{\imath}} \nu_{h_{i}}\right|\right)\left(\prod_{i \in S_{\delta}}\left|\delta_{h_{i}}\right|\right),
\end{aligned}
$$

where $Q_{v}^{j}$ is defined analogously to $P_{v}^{j}$ and

$$
\begin{aligned}
& J\left(\tau, P_{v_{0}}, \underline{x}_{v_{0}}\right)=\prod_{v \text { not e p }}\left(\xi_{v}-\xi_{v}^{\prime}\right)^{\bar{z}_{v}} \\
& \times \frac{1}{s_{v} !} \sum_{T_{v}} \int d \underline{r}^{\left(P_{v}\right)} e^{-\kappa \gamma^{h}} d_{T_{v}}^{*}\left(P_{v^{1}} \backslash Q_{v^{1}}, \quad, P_{v^{s}} \backslash Q_{v^{s}}\right)
\end{aligned}
$$


In Appendix 3 we prove also that

$$
\begin{aligned}
& \int d \underline{x}_{v_{0}} J\left(\tau, P_{v_{0}}, \underline{x}_{v_{0}}\right) e^{\bar{\kappa} \gamma^{k} d\left(P_{v_{0}}\right)} \\
& \leq|\Lambda| \prod_{v \text { not e p }} C^{\sum_{\imath}\left(\left|P_{v^{2}}\right|-\left|Q_{v^{2}}\right|\right)} \gamma^{-2 h_{v}\left(s_{v}-1\right)-h_{v} \bar{z}_{v}}
\end{aligned}
$$

if

$$
\bar{\kappa}<\frac{1}{2} \kappa\left(1-\gamma^{-1}\right)
$$

Note that in the bound (5.60) we took into account also the sum over the $\omega$ 's, giving at worst an extra factor $2^{4 n}$.

The bounds (5.58) and (5.60) imply that

$$
\begin{aligned}
& \frac{1}{|\Lambda|} \int d \underline{x}_{v_{0}} e^{\bar{\kappa} \gamma^{k} d\left(P_{v_{0}}\right)}\left|V^{(k)}\left(\tau, P_{v_{0}}, \underline{x}_{v_{0}}\right)\right| \\
& \leq \gamma^{-k D\left(P_{v_{0}}\right)} \sum_{\left\{P_{v}\right\}} \prod_{v \text { not e p }}\left|\frac{Z_{h_{v}}}{Z_{h_{v}-1}}\right|^{\frac{1}{2}\left|P_{v}\right|} \gamma^{-D\left(P_{v}\right)} C^{\sum_{\imath}\left|P_{v^{2}}\right|-\left|P_{v}\right|} \\
& \times\left(\prod_{i \in S_{\lambda}}\left|\lambda_{h_{\imath}}\right|\right)\left(\prod_{i \in S_{\nu}}\left|\nu_{h_{\imath}}\right|\right)\left(\prod_{i \in S_{\delta}}\left|\delta_{h_{\imath}}\right|\right)
\end{aligned}
$$

Remarking that, if $v \neq v_{0}, D\left(P_{v}\right)>0$ (the $\mathscr{R}$ operation was defined so as to obtain this result, see $[\mathrm{BG}]$ ); furthermore, we have

$$
D\left(P_{v}\right) \geq \frac{1}{6}\left|P_{v}\right|
$$

Then, by using also (5.51), we find, if $\frac{1}{2} c_{2} \bar{\varepsilon}^{2}<1 / 6-1 / 8$,

$$
\begin{gathered}
\gamma^{k D\left(P_{v_{0}}\right)} \int d \underline{x}_{v_{0}} e^{\bar{\kappa} \gamma^{k} d\left(P_{v_{0}}\right)}\left|V^{(k)}\left(\tau, P_{v_{0}}, \underline{x}_{v_{0}}\right)\right| \\
\leq\left(C \varepsilon_{k}\right)^{n} \sum_{\left\{P_{v}\right\}} \prod_{\substack{v \geq v_{0} \\
v \text { not e p }}} \gamma^{-\frac{1}{8}\left|P_{v}\right|},
\end{gathered}
$$

and this shows that the leading terms in the estimate are given by the contributions from the trees without trivial vertices (note that such trees are "concentrated" near the infrared scales in the sense that all their frequencies are between $k+1$ and $k+n$ : this happened also in the u.v. case of Sect. 3 but for a somewhat different mechanism).

We can now proceed as in the ultraviolet case and show that actually

$$
\sum_{\tau \in \mathscr{T}_{n}} \sum_{\left\{P_{v}\right\}} \prod_{v \text { not e } \mathrm{p}} \gamma^{-\frac{1}{8}\left|P_{v}\right|} \leq C^{n},
$$

ending the proof of the bound (5.52) and of the above theorem.

Remarks. 1) The bounds (5.64) can be easily converted into bounds on the functional derivatives

$$
\frac{\delta}{\delta \psi_{x}^{+}} V^{(h)}\left(\sqrt{Z_{h}} \psi\right)=\sqrt{Z_{h}} V_{x+}^{(h)^{\prime}}\left(\sqrt{Z_{h}} \psi\right)
$$


where, by (5.32), (5.33), and (5.46),

$$
\begin{aligned}
V_{x+}^{(h)^{\prime}}\left(\sqrt{Z_{h}} \psi\right)= & \sum_{n=1}^{\infty} \sum_{\tau \in \mathscr{T}_{n}} \sum_{P_{v_{0}}} \sum_{f \in P_{v_{0}}^{+}} \int d \underline{x}^{\left(P_{v_{0}}\right)} W^{(h)}\left(\tau, P_{v_{0}}, \underline{x}^{\left(P_{v_{0}}\right)}\right) \\
& \times Z^{\frac{1}{2}\left(\left|P_{v_{0}}\right|-1\right)} \partial^{m_{f}} \delta\left(x_{f}-x\right) \tilde{\psi}^{(\leq h)}\left(P_{v_{0}} \backslash f\right),
\end{aligned}
$$

$P_{v_{0}}^{+}$being the set of field labels associated with fields of type $\psi^{+}$or $\partial \psi^{+}$in $\tilde{\psi}^{(\leq h)}\left(P_{v_{0}}\right)$.

The functional derivative will be used in next section to study the Schwinger functions and we should get in trouble with the representation (5.67), unless there are no terms with $m_{f}>0$ in the r.h.s. Of course the definitions used so far do not imply such a property; however we could easily change them so that the field of label $f$ selected in (5.67) always appears [in $\tilde{\psi}^{(\leq h)}\left(P_{v_{0}}\right)$ ] exactly in the form $\psi_{x}^{+}$, without any derivative acting on it. This is achieved by considering the path $\mathscr{S}$ on the tree joining the root to the top vertex $v_{f}$, whose graph element contains the selected field of label $f$, and undoing all the $\mathscr{R}$ operations, acting in the vertices of $\mathscr{S}$ and involving subgraphs with four external lines. Then we recombine the various terms by using a new localization operation consisting in choosing as localization point always $x$ (i.e. we do not use the localization prescription of Sect. 4 in which the two $\psi^{+}$fields in a four external line subgraph are treated symmetrically: this means eliminating the factor $1 / 2$ in (4.12) and keeping only one of the two addends in the first line of (4.12), and precisely the first if $x_{1}=x$ or the second if $x_{2}=x$ ). The new prescription does not affect the running coupling constants, by symmetry reasons, as the two terms in (4.12) produce the same contributions to the running couplings.

It will be useful in the following section to have a bound of the kernels of (5.67) analogous to (5.52). We consider the contribution to $\delta V^{(h)} / \delta \psi_{x}^{+}$coming from a monomial containing $\left|P_{v_{0}}^{j+}\right|$ fields of type $\partial^{\jmath} \psi, j=0,1,2$, and look for the part of degree $g$ in the running coupling constants. We immediately get the bound:

$$
\begin{aligned}
& \sqrt{Z_{h}} \int d \underline{x}^{\left(P_{v_{0}}\right.} \sum_{\tau \in \mathcal{T}_{g}}\left|W^{(h)}\left(\tau, P_{v_{0}}, \underline{x}^{\left(P_{v_{0}}\right)}\right)\right| \delta\left(x_{f}-x\right) e^{\bar{\kappa} \gamma^{h} d\left(P_{v_{0}}\right)} \\
& \leq(C \varepsilon)^{g} \sqrt{Z_{h}} \gamma^{-\frac{1}{2} h} \gamma^{2 h} \gamma^{-\frac{h}{2} \sum_{\jmath}(2 j+1)\left|P_{v_{0}}^{j+}\right|}
\end{aligned}
$$

Note the factor $\sqrt{Z_{h}} \gamma^{-h / 2}$ to be associated with the field selected by the functional derivative.

2) Note that the above arguments do not hold for the functional derivatives with respect to $\psi_{x}^{-}$. The reason is simply that fields $\partial^{m} \psi_{x}^{-}, m>0$, arise also in the $\mathscr{R}$ and $\mathscr{L}^{*}$ operations on second order monomials.

Of course, however, the role of $\psi^{+}$and $\psi^{-}$is symmetric. This means that the same bounds hold for the functional derivatives of $V^{(h)}$ with respect to $\psi_{y}^{-}$!

A way to check explicitly the latter (obvious) statement would be to do once more the whole theory so far developed, by exchanging the role of $\psi^{+}$and $\psi^{-}$. Hence one would start by writing the kinetic part with the laplacian operating on the $\psi^{+}$field and so on, and in particular the localization operations would have to be defined by localizing over the points corresponding to the $\psi^{-}$fields. 


\section{The Two Point Schwinger Function}

As discussed in Sect. 2, in order to study the Schwinger functions (our results are summarized in the theorem at the end of this section), one has to calculate $V_{\text {eff }}(\varphi)$, which is related to the effective potential $\bar{V}^{(0)}$ by the relation (recall that $Z_{0}=1$ ),

$$
e^{-V_{\text {eff }}(\varphi)}=\frac{1}{\mathscr{N}^{c}} \int P_{Z_{0}}\left(d \psi^{(\leq 0)}\right) e^{-\bar{V}^{(0)}\left[\sqrt{Z_{0}}\left(\psi^{(\leq 0)}+\varphi\right)\right]} .
$$

By using (4.7) and the formal change of variables $\varphi+\varphi \rightarrow \psi$ (to be correctly interpreted as in Sect. 2), one can easily check that

$$
\begin{aligned}
e^{-V_{\mathrm{eff}}(\varphi)}= & \frac{1}{\mathscr{N}^{\cdot}} \int P_{Z_{0}}\left(d \psi^{(\leq 0)}\right) \\
& \times e^{-\bar{V}^{(0)}\left(\sqrt{Z_{0}} \psi\right)-\left(\varphi^{+}, C_{0} g^{-1} \varphi^{-}\right)+\left(\psi^{+}, C_{0} g^{-1} \varphi^{-}\right)+\left(\varphi^{+}, C_{0} g^{-1} \psi^{-}\right)},
\end{aligned}
$$

where $\psi=\psi^{(\leq 0)}, C_{0}$ is the convolution operator defined by (5.3) and $g^{-1}$ is the differential operator $\partial_{t}+e\left(i \partial_{\vec{x}}\right)$.

By using (2.33), we find

$$
q(\varphi)=\left(\varphi^{+},\left(1-C_{0}\right) g \varphi^{-}\right)+q^{(\leq 0)}\left(C_{0} \varphi\right)
$$

with the functional $q^{(\leq 0)}(\varphi)$ defined by

$$
e^{q^{(\leq 0)}(\varphi)}=\frac{1}{\mathscr{N}^{\cdot}} \int P_{Z_{0}}\left(d \psi^{(\leq 0)}\right) e^{-\bar{V}^{(0)}\left(\sqrt{Z_{0}} \psi\right)+\left(\psi^{+}, \varphi^{-}\right)+\left(\varphi^{+}, \psi^{-}\right)}
$$

Equation (6.3) implies a simple relation between the two point Schwinger function $S(x-y)$ and $S^{(\leq 0)}(x-y)=\delta^{2} q^{(\leq 0)}(\varphi) /\left.\delta \varphi_{x}^{+} \delta \varphi_{y}^{-}\right|_{\varphi=0}$, that is, in terms of Fourier transforms,

$$
\hat{S}(k)=\frac{1-e^{\left[k_{0}^{2}+e\left(k_{1}\right)^{2}\right] p_{0}^{-2}}}{-i k_{0}+e\left(k_{1}\right)}+S^{(\leq 0)}(k) e^{2\left[k_{0}^{2}+e\left(k_{1}\right)^{2}\right] p_{0}^{-2}}
$$

which means that, if we are interested in the infrared behaviour of the theory [i.e. $k_{0}$, e $(\vec{k})$ small), it is sufficient to study $q^{(\leq 0)}(\varphi)$, as we shall do in the following. We could study directly $q(\varphi)$, by the same technique discussed below, obtaining in this way information also on the ultraviolet behaviour of the two point Schwinger function; we would find results in agreement with the discussion of Sect. 3.

In order to study $S^{(\leq 0)}(\varphi)$, we shall use a tree expansion similar to that used in Sect. 5 , by suitably taking into account the new terms, linear in the external field $\varphi$, which are added in (6.4) to the effective potential $\bar{V}^{(0)}$.

The expansion is generated inductively, as in Sect. 5, by integrating step by step the fields of decreasing frequency index. We shall suppose again, for simplicity, that only the local terms are present in $\bar{V}^{(0)}$. The first step will be the integration of the field of frequency index $h=0$ in (6.4); we obtain the identity

$$
S^{(\leq 0)}=\frac{1}{Z_{0}} \tilde{g}^{(0)}+\frac{1}{Z_{0}} \tilde{g}^{(0)} * K_{2}^{(-1)} * \frac{1}{Z_{0}} \tilde{g}^{(0)}+S^{(\leq-1)}
$$

where $*$ denotes convolution, $K_{2}^{(-1)}$ is $Z_{0}$ times the kernel of $\tilde{V}_{2}^{(-1)}(\psi)$ [i.e. it is the kernel of $\tilde{V}_{2}^{(-1)}\left(\sqrt{Z_{0}} \psi\right)$ as a functional of $\left.\psi\right]$ and $S^{(\leq 1)}(x-y)=$ $\delta^{2} q^{(\leq-1)}(\varphi) /\left.\delta \varphi_{x}^{+} \delta \varphi_{y}^{-}\right|_{\varphi=0}$, with $q^{(\leq-1)}(\varphi)$ defined by

$$
e^{q^{(\leq-1)}(\varphi)}=\frac{1}{\mathscr{N}^{\prime \prime}} \int P_{Z_{-1}}(d \psi) e^{-V^{(-1)}\left(\sqrt{Z_{-1}} \psi\right)-\bar{W}^{(-1)}(\varphi, \psi)}
$$


Here $P_{Z_{-1}}(d \psi)$ is an abbreviation for $P_{Z_{-1}}\left(d \psi^{(\leq-2)}\right) \tilde{P}_{Z_{-1}}\left(d \psi^{(-1)}\right)$, see (5.18), and:

$$
\begin{aligned}
\bar{W}^{(-1)}(\varphi, \psi)= & \left(\psi^{+}, Q_{0} \varphi^{-}\right)+\left(\varphi^{+}, Q_{0} \psi^{-}\right) \\
& +\left[\varphi^{+} * G_{-1} * \sqrt{Z_{0}} \tilde{V}_{x+}^{(-1)^{\prime}}\right]\left(\sqrt{Z_{0}} \psi\right) \\
& +\left[\sqrt{Z_{0}} \tilde{V}_{y-}^{(-1)^{\prime}} * G_{-1} * \varphi^{-}\right]\left(\sqrt{Z_{0}} \psi\right) \\
& +\left[\varphi^{+} * G_{-1} * Z_{0} \tilde{V}_{\geq 2}^{(-1)^{\prime \prime}} * G_{-1} * \varphi^{-}\right]\left(\sqrt{Z_{0}} \psi\right) \\
& \times+\bar{W}_{R}^{(-1)}(\varphi, \psi)
\end{aligned}
$$

where

$$
Q_{0}=1, \quad G_{-1}=\frac{1}{Z_{0}} \tilde{g}^{(0)} * Q_{0}
$$

and we used for $\tilde{V}_{x \pm}^{(-1)^{\prime}}$ a definition analogous to (5.66) and $\tilde{V}_{\geq 2}^{(-1)^{\prime \prime}}$ represents the terms of the second functional derivative of $\tilde{V}^{(-1)}$ with two or more external legs; moreover $\bar{W}_{R}^{(-1)}(\varphi, \psi)$ represents the terms which do not contribute to $S^{(\leq-1)}(x-y)$ [because either they are of order $\varphi^{3}$ or they contain a factor $\left(\varphi^{+}\right)^{2}$ or $\left(\varphi^{-}\right)^{2}$ ].

In order to use the bounds on the functional derivative, that we found at the end of Sect. 5, we have to write (6.8) in terms of $V^{(-1)}$ instead of $\tilde{V}^{(-1)}$. Therefore we localize $\tilde{V}^{(-1)}$ and then we extract the local terms proportional to $\left[\partial_{t}+e\left(i \partial_{\vec{x}}\right)\right]$.

The terms proportional to $\left[\partial_{t}+e\left(i \partial_{\vec{x}}\right)\right]$ can be conveniently added to the terms $\left(\psi^{+}, Q_{0} \varphi^{-}\right)$and $\left(\varphi^{+}, Q_{0} \psi^{-}\right)$, so obtaining the following representation of (6.8):

$$
\begin{aligned}
\bar{W}^{(-1)}(\varphi, \psi)= & \left(\psi^{+}, Q_{-1} \varphi^{-}\right)+\left(\varphi^{+}, Q_{-1} \psi^{-}\right) \\
& +\left[\varphi^{+} * G_{-1} * \sqrt{Z_{-1}} V_{x+}^{(-1)^{\prime}}\right]\left(\sqrt{Z_{-1}} \psi\right) \\
& +\left[\sqrt{Z_{-1}} V_{y-}^{(-1)^{\prime}} * G_{-1} * \varphi^{-}\right]\left(\sqrt{Z_{-1}} \psi\right) \\
& +\left[\varphi^{+} * G_{-1} * Z_{0} \tilde{V}_{\geq 2}^{(-1)^{\prime \prime}} * G_{-1} * \varphi^{-}\right]\left(\sqrt{Z_{0}} \psi\right) \\
& \times+\bar{W}_{R}^{(-1)}(\varphi, \psi)
\end{aligned}
$$

where

$$
\begin{gathered}
Q_{-1}=Q_{0}-z_{-1} Z_{0}\left[\partial_{t}+e\left(i \partial_{\vec{x}}\right)\right] G_{-1}=Q_{0}-z_{-1} w_{0} * Q_{0} \\
w_{0}=\left[\partial_{t}+e\left(i \partial_{\vec{x}}\right)\right] \tilde{g}^{(0)}
\end{gathered}
$$

Note that no localization operation is performed on $\tilde{V}^{(-1)^{\prime \prime}}$.

The construction can be iterated and, at each step, we get new contributions to the two point Schwinger function, as in (6.6). We build in this way an expansion for $S^{(\leq 0)}(x-y)$ of the following type:

$$
S^{(\leq 0)}(x-y)=\sum_{h=-\infty}^{0} \sum_{k=-\infty}^{h-1} \sum_{n=0}^{\infty} \sum_{\tau \in \pi_{n}^{h, k}} S_{h, k, \tau}(x-y)
$$


Fig. 9.

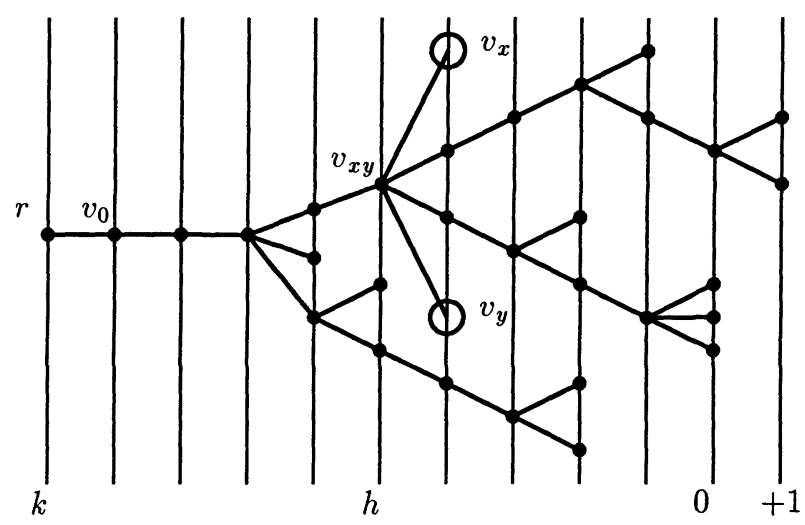

where the family of labeled trees $\mathscr{T}_{n}^{h, k}$ can be described as in Sect. 5, with the following modifications (see Fig. 9).

1) There are $n+2$ endpoints, $n \geq 0$, and two of them, denoted $v_{x}$ and $v_{y}$ in the figure, represent the following functions:

$$
\begin{aligned}
& \int d x \varphi_{x}^{+}\left[Q_{h} * \psi_{x}^{(\leq h)-}+G_{h} * \frac{\delta}{\delta \psi_{x}^{+}}\left(V^{(h)}\left(\sqrt{Z_{h}} \psi\right)\right)\right] \\
& \int d y\left[\psi_{y}^{(\leq h)+} * Q_{h}+\frac{\partial}{\delta \psi_{y}^{-}}\left(V^{(h)}\left(\sqrt{Z_{h}} \psi\right)\right) * G_{h}\right] \varphi_{y}^{-}
\end{aligned}
$$

where the following recursive relations for the convolution operators $Q_{h}, G_{h}$, hold:

$$
\begin{aligned}
Q_{h-1} & =Q_{h}-z_{h-1} Z_{h}\left[\partial_{t}+e\left(i \partial_{\vec{x}}\right)\right]\left[G_{h}+\frac{1}{Z_{h}} \tilde{g}^{(h)} * Q_{h}\right] \\
& =Q_{h}-z_{h-1} \sum_{\jmath=h}^{0} \frac{Z_{h}}{Z_{j}} w_{j} * Q_{j}, \quad Q_{0}=1, \\
G_{h-1} & =G_{h}+\frac{1}{Z_{h}} \tilde{g}^{(h)} * Q_{h}=\sum_{j=h}^{0} \frac{1}{Z_{j}} \tilde{g}^{(j)} * Q_{j}, \quad G_{0}=0, \\
w_{h} & =\left[\partial_{t}+e\left(i \partial_{\vec{x}}\right)\right] \tilde{g}^{(h)} .
\end{aligned}
$$

2) The two special endpoints of item 1) belong to the vertical line with frequency index $h+1$ and are attached at the same tree vertex $v_{x y}$ bearing a frequency label $h$. This implies that $h$ is the scale at which the lines $\varphi_{x}^{+}$and $\varphi_{y}^{-}$become connected by graph lines.

3) There are no external lines in the root of the tree.

4) There are no $\mathscr{R}$ labels associated with the tree vertices $v$ belonging to the line $\mathscr{F}$ joining the root to $v_{x y}$.

In the quasi-particel representation (which is used for the bounds) the renormalized propagator $G_{h}(x)$ can be written as

$$
G_{h}(x)=\sum_{\omega} G_{h, \omega}(x) e^{-i p_{F} \omega \vec{x}}, \quad G_{h, \omega}(x)=\sum_{j=h+1}^{0} \frac{1}{Z_{\jmath}} \tilde{g}_{Q, \omega}^{(\jmath)}(x)
$$


where $\tilde{g}_{Q, \omega}^{(j)}(x)$ has a definition similar to that of $\tilde{g}_{\omega}^{(j)}(x)$, see (5.9) and (4.4), that is

$$
\tilde{g}_{Q, \omega}^{(h)}(x)=e^{i p_{F} \omega \vec{x}} \int \frac{d k}{(2 \pi)^{2}} e^{-i k x} \tilde{g}^{(h)}(k) Q_{h}(k) \chi\left(\omega \gamma^{-h} \vec{k}\right)
$$

In Appendix 1 we show that $\tilde{g}_{Q, \omega}^{(h)}(x)$ satisfies a bound like (5.27):

$$
\left|\tilde{g}_{Q, \omega}^{(h)}(x)\right| \leq C \gamma^{h} e^{-\kappa^{\prime} \gamma^{h}|x|}
$$

for any $\kappa^{\prime}<\kappa$, provided the $z_{h}$ verify $\left|z_{h}\right| \leq C \varepsilon^{2}$ for all $h$, with $\varepsilon$ small enough (i.e., by the bounds of Sect. 5, provided the running couplings $r_{h}$ verify $\left|r_{h}\right|<\varepsilon$ for $\varepsilon$ small enough).

Hence, for the purpose of establishing bounds in $x$ space, we could replace $Q_{h}$ by 1. It is possible to prove that a similar property is valid in $k$ space, but we shall not give the details.

Equation (6.17) easily implies that, for any $\bar{\kappa}<\kappa$,

$$
\int d x e^{\bar{\kappa} \gamma^{h}|x|}\left|G_{h, \omega}(x)\right| \leq C^{\prime} \frac{\gamma^{-h}}{Z_{h}}
$$

We want to show that

$$
\sum_{k=-\infty}^{h-1} \sum_{\tau \in \mathscr{T}_{n}^{h, k}}\left|S_{h, k, \tau}(x-y)\right| \leq(C \varepsilon)^{n} \frac{\gamma^{h}}{Z_{h}} e^{-\bar{\kappa} \gamma^{h}|x-y|}
$$

We shall treat explicitly only the bound of the contributions to the values of the tree in Fig. 9, coming from the second terms in (6.13) only; the other three possibilities can be (more easily) treated along the same lines. For such contributions we can write

$$
S_{h, k, \tau}(x-y)=\sum_{\omega \omega^{\prime}} \int d x_{0} d y_{0} G_{h, \omega}\left(x-x_{0}\right) \bar{S}_{h, k, \tau}\left(x_{0}-y_{0}\right) G_{h, \omega^{\prime}}\left(y_{0}-y\right) .
$$

By (6.18), it is sufficient to show that

$$
\sum_{k=-\infty}^{h-1} \sum_{\tau \in \mathcal{F}_{n}^{h}, k}\left|\bar{S}_{h, k, \tau}\left(x_{0}-y_{0}\right)\right| \leq(C \varepsilon)^{n} Z_{h} \gamma^{3 h} e^{-\bar{\kappa} \gamma^{h}\left|x_{0}-y_{0}\right|}
$$

Note that, if we are interested in the contribution of order $m$ in the running coupling constants, we have to pick out of (6.13) the terms of order $n_{ \pm}=0,1, \ldots$ and consider trees with $n=m-n_{+}-n_{-}$.

The remarks at the end of Sect. 5 and the bound (5.68) play a key role. In order to prove $(6.21)$ we have to consider the contributions to the functional derivatives $\delta V^{(h)} / \delta \psi_{x_{0}}^{+}$and $\delta V^{(h)} / \delta \psi_{y_{0}}^{-}$coming from the monomials containing $\left|P_{v_{x y}}^{J \pm}\right|$ fields of $\partial^{j} \psi$-type and of degree $n_{ \pm}$in the running coupling constants. In this way we expand 
$\bar{S}_{h, k, \tau}\left(x_{0}-y_{0}\right)$ in a sum of term, that we can bound proceeding as in Sect. 5. We obtain:

$$
\begin{aligned}
& {\left[(C \varepsilon)^{n_{+}} \sqrt{Z_{h}} \gamma^{-\frac{1}{2} h} \gamma^{2 h} \gamma^{-\frac{h}{2} \sum_{\jmath}(2 j+1)\left|P_{v_{x}}^{\jmath+}\right|}\right]} \\
& \quad \times\left[(C \varepsilon)^{n_{-}} \sqrt{Z_{h}} \gamma^{-\frac{1}{2} h} \gamma^{2 h} \gamma^{-\frac{h}{2} \sum_{\jmath}(2 j+1)\left|P_{v y}^{\jmath-}\right|}\right] \\
& \quad \times(C \varepsilon)^{n}\left[\gamma^{2 h}\right] \prod_{\mathrm{vep}} \gamma^{h_{v} \delta_{2}(v)} \\
& \quad \times \prod_{v \text { not e p }}\left(\frac{Z_{h_{v}}}{Z_{h_{v}-1}}\right)^{\frac{1}{2}\left|P_{v}\right|} \gamma^{h_{v}\left(\frac{1}{2} \sum_{i, j}\left(\left|P_{v^{2}}^{J}\right|-\left|Q_{v^{i}}^{j}\right|\right)(2 j+1)-2\left(s_{v}-1\right)\right)},
\end{aligned}
$$

where the first two factors arise from the bound (5.68) and $\delta_{2}(v)=1$ if the end point of the tree represents a chemical potential running coupling $\nu_{h} \gamma^{h}$. The extra factor $\left[\gamma^{2 h}\right]$ in square brackets is there because there is no integration on $y_{0}$ but we count $s_{v}-1$ space integrations for all vertices, while there are really only $s_{v_{x y}}-2$ for $v=v_{x y}$ (recall that $h_{v_{x y}}=h$ in our notations).

From (6.22), after a power counting computation, we get:

$$
\begin{aligned}
& (C \varepsilon)^{n+n_{+}+n_{-}} Z_{h} \gamma^{3 h} e^{-\bar{\kappa} \gamma^{h}\left|x_{0}-y_{0}\right|} \\
& \quad \times \prod_{\substack{v \text { not e } \mathrm{p} \\
v \notin \nearrow}} \gamma^{-D\left(P_{v}\right)-\eta\left|P_{v}\right|} \prod_{v \in \zeta} \gamma^{-\sum_{\jmath}\left|P_{v}^{j}\right|\left(\frac{2 \jmath+1}{2}+\eta\right)},
\end{aligned}
$$

where we have written $\gamma^{-2 \eta}$, with

$$
2 \eta=\liminf _{h \rightarrow-\infty} \frac{\log Z_{h}}{|h|}
$$

instead of the correct value $Z_{h_{v}} Z_{h_{v}-1}^{-1}$ (asymptotic to it), to simplify the notations, and $D\left(P_{v}\right)$ is defined in Sect. 5, (5.53).

The bound (6.23) implies $(6.21)$ by the same arguments used in Sect. 5. The conclusion is that:

Theorem 3. The pair Schwinger function can be written in the form

$$
\sum_{h=-\infty}^{0} \frac{1}{Z_{h}}\left(g^{(h)}+\varepsilon \bar{g}^{(h)}\right),
$$

where $\varepsilon$ is supposed to be small enough and to be a bound on the running couplings on all scales, and

$$
\left|\bar{g}^{(h)}(x-y)\right| \leq B \gamma^{h} e^{-\bar{\kappa} \gamma^{h}|x-y|},
$$

$B<0$ being a suitable constant, independent on $\varepsilon$. Furthermore, under the same conditions, $S(x-y)$ is analytic in the running couplings with a domain independent on $x-y$.

An immediate corollary of the theorem is that the pair Schwinger function decays, for $|x-y| \rightarrow \infty$, as $|x-y|^{-1-2 \eta}$, with $\eta$ defined by (6.24), if the sequence in the 
r.h.s. is convergent, as the analysis of the following section implies. Furthermore, by explicit calculation it is easy to prove that $\eta=c \lambda_{-\infty}^{2}+O\left(\varepsilon^{3}\right)$, with $c>0$. In Sect. 7 we shall also prove that the running couplings are analytic functions of $\lambda_{0}$ near 0 ; hence since the analysis of Sect. 3 implies that $\lambda_{0}$ is analytic in $\lambda$, we have, using (4.41):

$$
\eta=c\left[2 \lambda\left(\hat{v}(0)-\hat{v}\left(2 p_{F}\right)\right)\right]^{2}+O\left(\varepsilon^{3}\right) .
$$

\section{The Vanishing of the Beta Function and Completion of the Theory of Spinless Fermi Systems}

It remains to prove that there is a small $\varepsilon$ such that $\left|\lambda_{h}\right|,\left|\delta_{h}\right|,\left|\nu_{h}\right|<\varepsilon, \forall h$, if the initial coupling constant $\lambda$ is small enough and the parameters $\alpha$ and $\nu$ [see (1.3)] are suitably chosen. This was conjectured in [BG] on the basis of heuristic arguments; some further heuristic arguments for the proof of such a statement were presented also in [BGM]. Here we want to reduce the proof to some technical lemma, which we think are easy consequences of the analysis of Sect. 5, of the results of [GS], and of some properties of the exact solution of the Luttinger model (see [ML] and $[B G M])$. A more detailed proof will probably be published elsewhere as it would make this paper too long, but we think that it is not really necessary as all the steps are clearly outlined below referring to the estimates of the previous sections, and no further information is needed.

A direct proof of the boundedness of the running coupling constants might also be possible, by using the symmetry properties of the propagators (see [S, DM] for a heuristic discussion), but we met serious obstacles in trying to do it, although we succeeded in proving the key property (7.6) below (i.e. the vanishing of the beta function in the scaling limit) to fourth order and to see several cancellations to all orders.

Let us call $\mu_{h}=\left(\lambda_{h}, \delta_{h}\right)$; by eliminating the $Z_{h}$ constants from the r.h.s. of Eqs. (5.48) through (5.47) and using the theorem and the remark following them in Sect. 5 , it is possible to prove that we can rewrite the beta functional as

$$
\begin{aligned}
& \mu_{h-1}=\mu_{h}+B^{h, \mu}\left(\mu_{h}, \nu_{h}, \mu_{h+1}, \nu_{h+1}, \ldots, \mu_{0}, \nu_{0}\right), \\
& \nu_{h-1}=\gamma \nu_{h}+B^{h, \nu}\left(\mu_{h}, \nu_{h}, \mu_{h+1}, \nu_{h+1}, \ldots, \mu_{0}, \nu_{0}\right),
\end{aligned}
$$

where the $B^{h}$ are analytic in $\mu_{h^{\prime}}, \nu_{h^{\prime}}, h^{\prime} \geq h$, if $\left|\mu_{h^{\prime}}\right|,\left|\nu_{h^{\prime}}\right|<\varepsilon$, for a suitable small $\varepsilon$.

The property $\gamma>1$ can be used to show that the above relation is equivalent to

$$
\begin{aligned}
& \mu_{h-1}=\mu_{h}+\mathscr{B}_{\mu}^{h}\left(\mu_{h}, \ldots, \mu_{0} ; \nu_{h}\right), \\
& \nu_{h-1}=\gamma \nu_{h}+\mathscr{B}_{\nu}^{h}\left(\mu_{h}, \ldots, \mu_{0} ; \nu_{h}\right)
\end{aligned}
$$

with $\mathscr{B}^{h}$ analytic for $\left|\mu_{h^{\prime}}\right|<\varepsilon, h^{\prime} \geq h$, and $\left|\nu_{h}\right|<\varepsilon$. See Appendix 4 for the proof.

By direct calculation one checks also that:

$$
\mathscr{B}_{\nu}^{h}\left(\mu_{h}, \ldots, \mu_{0} ; \nu_{h}\right)=\nu_{h} \lambda_{h}^{2} \mathscr{B}^{\prime h}\left(\mu_{h}, \ldots, \mu_{0} ; \nu_{h}\right)+\gamma^{h} \mathscr{B}^{\prime \prime h}\left(\mu_{h}, \ldots, \mu_{0} ; \nu_{h}\right)
$$

with $\left|\mathscr{B}^{\prime h}\right| \leq C,\left|\mathscr{B}^{\prime \prime h}\right| \leq C \varepsilon^{2}$ for a suitable $C$ and for $\varepsilon$ small enough, see [BGM].

The relations (7.2), (7.3), given any infinite sequence $\mu_{h}$ with $\left|\mu_{h}\right|<\varepsilon$, imply that there is a unique $\nu_{0}$ such that $\left|\nu_{h}\right|<\varepsilon$ and $\nu_{h} \rightarrow 0$ as $h \rightarrow-\infty$, and $\nu_{0}$ is analytic in the running constants $\mu_{h}$ for $\left|\mu_{h}\right|<\varepsilon$; moreover the convergence to 0 will be at 
the rate $\nu_{h}=O\left(\gamma^{h}\right)$ (see [BG]). This is a version of the existence of an unstable manifold theorem.

Furthermore, since the analysis of Sect. 3 implies that $\nu_{0}$ is an analytic function of $\lambda, \alpha, \nu$, this value of $\nu_{0}$ is obtained, given $\alpha$ and $\lambda$, by a unique choice of $\nu$.

In [BG] it was shown that $\delta_{h-1}=\delta_{h}+O\left(\lambda_{h}^{2} \delta_{h}\right)$; hence, if $\lambda_{h}$ stays bounded away from zero, as $h \rightarrow-\infty$, one can apply the previous arguments to show that also $\delta_{0}$ can be chosen so that $\delta_{h} \rightarrow 0$, as $h \rightarrow-\infty$; this choice would fix also the value of $\alpha$. However, the following analysis shows that this choice is not necessary to control the flow of $\mu_{h}$, while of course the choice of $\nu_{0}$ is essntial.

Remark The previous considerations imply that we can consider the running couplings as functions of $\mu \equiv(\lambda, \alpha)$. If we also take into account the results of Sect. 3, we can claim that there is a small $\varepsilon_{0}$, such that, if for all $\mu$ with $|\mu| \leq \varepsilon_{0}$ (so that $\mu_{0}$ is well defined as an analytic function of $\mu$ and $\left.\left|\mu_{0}\right| \leq \varepsilon\right)$ it happens that $\left|\mu_{h^{\prime}}\right| \leq \varepsilon$ for $h^{\prime} \geq h$, then $\mu_{h^{\prime}}, h^{\prime} \geq h-1$, is holomorphic in $\mu$, for $|\mu| \leq \varepsilon_{0}$.

We want to show that the running couplings stay really bounded (and analytic in $\mu$ ) for all $h \leq 0$, if $|\mu| \leq \bar{\varepsilon} \leq \varepsilon_{0}$. In order to do that, we shall need the following function:

$$
\lim _{h \rightarrow-\infty} \mathscr{B}_{\mu, \mathscr{L}}^{h}(\bar{\mu}, \bar{\mu}, \ldots, \bar{\mu} ; 0) \equiv \mathscr{B}_{\mathscr{L}}(\vec{\mu})
$$

where $\mathscr{S}_{\mu, \mathscr{L}}^{h}$ is the beta function of Luttinger model, defined in a way entirely analogous to the above $\mathscr{B}_{\mu}^{h}$. Such a definition is rather delicate in the part concerning the ultraviolet cut-off (i.e. in the part corresponding to the contents of Sect. 3) but it has been discussed in detail in [GS]. The part concerning the infrared cut-off requires an analysis identical to the one just carried out (this was pointed out in [BG, BGM]); such analysis and the fact that $\nu_{h}=O\left(\gamma^{h}\right)$ also imply that:

$$
\begin{aligned}
\mathscr{B}_{i}^{h}\left(\mu_{h}, \mu_{h+1}, \ldots, \mu_{0} ; \nu_{h}\right)= & \mathscr{B}_{i, \mathscr{L}}^{h}\left(\mu_{h}, \mu_{h+1}, \ldots, \mu_{0} ; 0\right) \\
& +\gamma^{h} R_{i}^{h}\left(\mu_{h}, \mu_{h+1}, \ldots, \mu_{0} ; \nu_{h}\right),
\end{aligned}
$$

where $i=\mu, \nu$ and $R^{h}$ has the same structure and satisfies the same bounds as $\mathscr{B}^{h}$. This essentially follows from the observation that the single scale propagator $g_{\omega}^{(h)}(x)$ [see (4.4)] differs from the analogous Luttinger model propagator [obtained by linearizing $e(\vec{k})$ around $\vec{k}=\omega p_{F}$ ] by terms of order $\gamma^{h}$, and exponentially decaying in $\gamma^{h}|x|$ (see [BG]).

Furthermore the function $\mathscr{B}_{\nu, \mathscr{E}}^{h}\left(\mu_{h}, \ldots, \mu_{0} ; 0\right)$ vanishes because of the special symmetries of the Luttinger model, see [BGM], i.e. in such case the unstable manifold is the plane $\nu=0$.

The main point of our analysis will be the proof that, in the Luttinger model (with $\nu=0$, see above), the running couplings stay bounded for all $h \leq 0$, if $\mu$ is small enough. From this property we shall deduce the strong property:

$$
\mathscr{B}_{\mathscr{G}}(\bar{\mu})=0, \text { for all small } \bar{\mu} \text {. }
$$

The latter equality will, in turn, be used to prove that the running couplings are bounded also in our model.

We start by remarking that the Luttinger model is exactly soluble, even if the particles are constrained in a finite space box of size $L$, with periodic boundary conditions, $[\mathrm{ML}]$. Furthermore the analysis of the previous sections and the results of [GS] could be applied to the model in finite volume without any uniformity problem, and we would get bounds uniform in $L$. By some refinement of our techniques, we 
can also prove a "continuous $L$-dependence" of the running couplings in the following sense.

Let $\mu_{h}^{(L)}$ be the running couplings for the model in finite volume, while $\mu_{h}$ still denotes the infinite volume running couplings and let $\varepsilon$ be the radius of convergence of the beta function, independent of $L$; and we define $L_{h} \equiv \gamma^{-h} p_{0}^{-1}$.

Lemma 1. If $\mu_{h^{\prime}}$ is defined and $\left|\mu_{h^{\prime}}\right| \leq \tilde{\varepsilon} \leq \varepsilon / 2$, for $h^{\prime} \geq h$, then there exists $n_{0}>0$ such that also $\mu_{h^{\prime}}^{\left(L_{h-n}\right)}$ is defined for $h^{\prime} \geq h$ and for any positive integer $n \geq n_{0}$; furthermore

$$
\left|\mu_{h^{\prime}}^{\left(L_{h-n}\right)}-\mu_{h^{\prime}}\right| \leq b_{0} \tilde{\varepsilon}^{2} e^{-\kappa n}, \quad h^{\prime} \geq h
$$

for some positive constants $b_{0}$ and $\kappa$.

It is very easy to prove this statement at any order of perturbation theory (in the running couplings), by using the exponential decay of the single scale covariances (which makes very slightly dependent on $L$, for $L$ large, the integrals involved in the definition of the beta function) and the remark that $g_{\omega}^{(h, L)}-g_{\omega}^{(h)}$ is of order $1 / L$. It is also easy to see that the completion of the proof rests on a "good" bound of the difference between the finite and infinite volume expectations of a generic monomial $\tilde{\psi}^{(h)}(P)$. In Appendix 2 we show that this "good" bound can be indeed obtained in a simple way.

Another key remark is that the finite volume acts as an infrared cut-off, so that the running couplings $\mu_{h}^{(L)}$ "stop" flowing after the scale corresponding to $L$ has been reached. This property can be formalized in the following lemma.

Lemma 2. There exists $\varepsilon_{1} \leq \varepsilon$, such that, for any fixed $h$, if $\mu_{h^{\prime}}^{\left(L_{h}\right)}$ is defined and $\left|\mu_{h^{\prime}}^{\left(L_{h}\right)}\right| \leq \tilde{\varepsilon} \leq \varepsilon_{1}$ for $h^{\prime} \geq h$, then $\mu_{h^{\prime}}^{\left(L_{h}\right)}$ is defined also for all $h^{\prime}<h$, and the limit $\mu_{-\infty}^{\left(L_{h}\right)}=\lim _{h^{\prime} \rightarrow-\infty} \mu_{h^{\prime}}^{\left(L_{h}\right)}$ does exist and

$$
\left|\mu_{h}^{\left(L_{h}\right)}-\mu_{-\infty}^{\left(L_{h}\right)}\right| \leq b_{1} \tilde{\varepsilon}^{2}
$$

for some constant $b_{1}$.

We do not give a formal proof of such a statement, quite obvious from the discussion of the preceding sections. In fact, once the scale $\gamma^{-h^{\prime}} p_{0}^{-1}$ becomes larger than the size $L_{h}$ of the container, the space integrations yield a factor proportional to $\gamma^{-h}$ rather than $\gamma^{-h^{\prime}}$; hence the beta function vanishes exponentially fast as $\gamma^{-\left(h-h^{\prime}\right)}$, when $h^{\prime} \leq h, h^{\prime} \rightarrow-\infty$.

Note that we are setting a cut-off only on the spatial part of the variables, while no cut-off is imposed on the time part (as we must, since the Luttinger model is exactly soluble only with no cut-off on the time variables). Setting a cut-off also on the time part would lead to an even faster approach to zero of the beta function $\left(\sim \gamma^{-2\left(h-h^{\prime}\right)}\right)$, but we could not compare the results with any exact calculation.

The last important remark is that it is possible to calculate $\mu_{-\infty}^{\left(L_{h}\right)}$, by using the explicit expressions of the Schwinger functions in the version of the Luttinger model studied in [BGM] (which is slightly different from the model solved in [ML], but it is more suitable for the comparison with the model studied in this paper).

In fact, in the presence of an infrared cut-off, $Z_{h}^{(L)}$ is not divergent for $h \rightarrow-\infty$; hence it is possible to define the non-anomalous effective potential through a relation similar to (2.33), by introducing an external field depending on $\omega$ (the Luttinger 
model is defined directly in terms of the quasi particle fields). The analysis of [BGM] immediately implies that we can write, if the spatial momenta are translated so that $p_{F}=0$,

$$
\begin{aligned}
V_{\mathrm{eff}}(\varphi)= & \text { const }+\sum_{\omega} \int d x d y V_{2}(x-y, \omega) \varphi_{\omega x}^{+} \varphi_{\omega y}^{-} \\
& +\int d x_{1} d x_{2} d x_{3} d x_{4} V_{4}\left(x_{1}, x_{2}, x_{3}, x_{4}\right) \\
& \times \varphi_{+1 x_{1}}^{+} \varphi_{-1 x_{2}}^{+} \varphi_{-1 x_{3}}^{-} \varphi_{+1 x_{4}}^{-}+O\left(\varphi^{6}\right) .
\end{aligned}
$$

Furthermore, it is possible to see that

$$
\begin{aligned}
& \frac{1}{\left(1+\delta_{-\infty}^{(L)}\right)} \frac{1}{Z_{-\infty}^{(L)}}=1-\int_{0}^{L} d \vec{x} \int d x_{0} i \omega \vec{x} s_{L} V_{2}(x, \omega), \\
& \frac{1}{\left(1+\delta_{-\infty}^{(L)}\right)^{2}} \frac{1}{Z_{-\infty}^{(L)}} \delta_{-\infty}^{(L)}=\int_{0}^{L} d \vec{x} \int d x_{0}\left(i \omega \vec{x} s_{L}+x_{0} c_{L}\right) V_{2}(x, \omega), \\
& \frac{1}{\left(1+\delta_{-\infty}^{(L)}\right)^{4}} \frac{1}{\left(Z_{-\infty}^{(L)}\right)^{2}} \lambda_{-\infty}^{(L)}=\int_{0} d x d x_{3} d x_{4} V_{4}\left(0, x, x_{3}, x_{4}\right) c_{L} c_{L}^{\prime} \\
& \text { where } s_{L}=\left(\frac{\pi \vec{x}}{L}\right)^{-1} \sin \frac{\pi \vec{x}}{L} \text { and } c_{L} \cos \frac{\pi \vec{x}}{L}, c_{L}^{\prime}=\cos \frac{\pi\left(\vec{x}_{3}-\vec{x}_{4}\right)}{L} .
\end{aligned}
$$

By (2.33) $V_{2}$ and $V_{4}$ are simply related to the two point and four point Schwinger functions, which are explicitly known, see [BGM], Eqs. (2.4), (2.5), and (2.9); hence $\mu_{-\infty}^{(L)}$ can be calculated and one can check, by a cumbersome analysis of the explicit expressions in terms of elementary functions of the integrands in (7.10), that (using the definitions of $[\mathrm{BGM}])$ :

Lemma 3. There exist $\varepsilon_{0}$, such that, if $|\mu| \leq \varepsilon_{0}$.

$$
\left|\mu_{-\infty}^{(L)}-\tilde{\mu}\right| \leq b_{2}|\mu|^{2}, \quad \text { for all } L>0
$$

for some constant $b_{2}$ and

$$
\tilde{\mu}=\left(\frac{\lambda}{2} \hat{v}(0), \alpha+\frac{\lambda}{4 \pi} \hat{v}(0)\right) .
$$

Let us now suppose that, given $\tilde{\varepsilon} \leq \varepsilon / 2$, there exists $h_{0}>-\infty$, such that

$$
\left|\mu_{h}\right| \leq \frac{\tilde{\varepsilon}}{2}<\left|\mu_{h_{0}}\right|<\tilde{\varepsilon}, \quad h>h_{0}
$$

Note that if $\left|\mu_{h^{\prime}}\right| \leq \tilde{\varepsilon} \leq \varepsilon, h^{\prime}>h$, then the bounds of Sect. 5 imply that

$$
\left|\mu_{h^{\prime}}^{(L)}-\mu_{h^{\prime}+1}^{(L)}\right| \leq b \tilde{\varepsilon}^{2}, \quad \text { for all } h^{\prime} \geq h
$$

for some positive $b$, independent of $L$.

We start with a small $\mu$, say $|\mu| \leq \bar{\varepsilon} \leq \frac{1}{4} \tilde{\varepsilon}$ and remark that $\mu_{h^{\prime}}$ stays close to the finite volume running couplings $\mu_{h^{\prime}}^{\left(L_{h_{0}}-n\right)}$ for $h^{\prime} \geq h_{0}:\left|\mu_{h^{\prime}}-\mu_{h^{\prime}}^{\left(L_{h_{0}}-n\right)}\right| \leq$ 
$b_{0} \tilde{\varepsilon}^{2} e^{-\kappa n} \leq \frac{1}{8} \tilde{\varepsilon}$ (see Lemma 1), having fixed once and for all $n$ to be such that the second inequality holds.

But we know that $\mu_{h_{0}}^{\left(L_{h_{0}-n}\right)}$ is close to $\mu_{h_{0}-n}^{\left(L_{h_{0}-n}\right)}$ by $2 b \tilde{\varepsilon}^{2} n$ [by (7.14)] (the factor 2 takes into account the small growth of $\mu_{h^{\prime}}^{\left(L_{h_{0}-n}\right)}$ for $\left.h^{\prime}<h_{0}\right)$; the latter is close to $\mu_{-\infty}^{\left(L_{\left.h_{0}-n\right)}\right)}$ by $b_{1} \tilde{\varepsilon}^{2}$, (by Lemma 2); and the latter is close to $\tilde{\mu}$ by $b_{2} \bar{\varepsilon}^{2}$ by Lemma 3 . Hence $\mu_{h_{0}}$ is close to $\tilde{\mu}$ by $b_{2} \bar{\varepsilon}^{2}+b_{1} \tilde{\varepsilon}^{2}+2 b \tilde{\varepsilon}^{2} n+\frac{1}{8} \tilde{\varepsilon}$. It is now sufficient to choose $\tilde{\varepsilon}$ small enough to conclude that

$$
\left|\mu_{h_{0}}\right| \leq \frac{1}{2} \tilde{\varepsilon}
$$

in contradiction with (7.13).

Remark. The above formal proof has a simple meaning. If, starting with $|\mu|<\bar{\varepsilon} \leq \tilde{\varepsilon} / 4$, it is nevertheless $\left|\mu_{h_{0}}\right|>\frac{1}{2} \tilde{\varepsilon} \geq 2 \bar{\varepsilon}$, this means that the running couplings can start arbitrarily small and reach size $O(1)$ [actually $O(\tilde{\varepsilon})$, as in this argument $\tilde{\varepsilon}$ has to be regarded fixed] in finitely many steps.

However the value that they reach is (Lemma 1) close to the value that they would reach in the theory with cut off at scale $L_{h_{0}}$ (Lemma 2). But by the exact solution, we know that such a value is still of $O(\bar{\varepsilon})$, hence it cannot be of size $O(1)$ (i.e. $>\tilde{\varepsilon} / 2$ ), and this is a contradiction.

The previous considerations can be summarized in the following theorem.

Theorem 4. In the infinite volume Luttinger model, for any $h \leq 0$, the running coupling $\mu_{h}$ is a well defined analytic function of $\mu$, if $|\mu| \leq \bar{\varepsilon}$, for a suitable $\bar{\varepsilon}$, and:

$$
\left|\mu_{h}-\tilde{\mu}\right| \leq C|\mu|^{2} \text {. }
$$

We are now ready to prove (7.6).

We can write:

$$
\begin{aligned}
\mathscr{B}_{\mu, \mathscr{L}}^{h}\left(\mu_{h}, \mu_{h+1}, \ldots, \mu_{0} ; 0\right)= & \mathscr{B}_{\mu, \mathscr{L}}^{h}\left(\mu_{h}, \mu_{h}, \ldots, \mu_{h} ; 0\right) \\
& +\sum_{k=h+1}^{0} D^{h, k}\left(\mu_{h}, \mu_{h+1}, \ldots, \mu_{0}\right),
\end{aligned}
$$

where

$$
\begin{aligned}
D^{h, k}\left(\mu_{h}, \mu_{h+1}, \ldots, \mu_{0}\right)= & \mathscr{S}_{\mu, \mathscr{L}}^{h}\left(\mu_{h}, \ldots, \mu_{h}, \mu_{k}, \mu_{k+1}, \ldots, \mu_{0} ; 0\right) \\
& -\mathscr{O}_{\mu, \mathscr{L}}^{h}\left(\mu_{h}, \ldots, \mu_{h}, \mu_{h}, \mu_{k+1}, \ldots, \mu_{0} ; 0\right) .
\end{aligned}
$$

From the analysis of Sect. 5, it is not difficult to deduce that:

$$
\mathscr{B}_{\mu, \mathscr{E}}^{h}\left(\mu_{h}, \mu_{h}, \ldots, \mu_{h} ; 0\right)=\mathscr{B}_{\mathscr{E}}\left(\mu_{h}\right)+O\left(\gamma^{h}\right)
$$

if, of course, $\left|\mu_{h}\right| \leq \tilde{\varepsilon}$ for all $h \leq 0$. The function $\mathscr{S}_{\mathscr{S}}(\bar{\mu})$ is holomorphic near $\bar{\mu}=0$ $(|\bar{\mu}| \leq \varepsilon)$.

Let us suppose that (7.6) is not true; hence there exists $r \geq 2$ such that

$$
\mathscr{B}_{\mathscr{S}}\left(\mu_{h}\right)=b_{r} \mu_{h}^{r}+O\left(\mu_{h}^{r+1}\right), \quad b_{r} \neq 0
$$

and in fact, by explicit calculation, one verifies that $r \geq 3$, see for instance [BG or BGM] for this (well known) fact. 
We want to show that this is in contradiction with Theorem 4 above and the structure of the beta function.

In fact, by Theorem 4 , if $|\mu| \leq \bar{\varepsilon}$,

$$
\mu_{h}=\tilde{\mu}+\sum_{n=2}^{r} c_{n}^{(h)} \mu^{n}+O\left(\mu^{r+1}\right)
$$

and for each fixed $n$ the sequence, labeled by $h \leq 0,\left\{c_{n}^{(h)}\right\}_{h \leq 0}$ is a bounded sequence.

Hence, if we insert the power expansions (7.21) in the first equation of (7.2) and in Eq. (7.17) and use (7.19), (7.20), we can write

$$
\sum_{n=2}^{r} c_{n}^{(h-1)} \mu^{n}=\sum_{n=2}^{r} c_{n}^{(h)} \mu^{n}+b_{r} \mu^{r}+\sum_{k=h+1}^{0} \sum_{n=3}^{r} d_{n}^{h, k} \mu^{n}+O\left(\gamma^{h}\right)
$$

where $\sum_{n=3}^{r} d_{n}^{h, k} \mu^{n}$ represents the Taylor expansion of $D^{h, k}$ up to order $r$.

The coefficients $d_{n}^{h, k}$ can be bounded by recalling the analysis of Sect. 5 . We see that for all complex $\mu$ 's, $|\mu|<\bar{\varepsilon}$, it is $D^{h, k}=\left(\mu_{h}-\mu_{k}\right) \bar{D}^{h, k}$ because $D^{h, k}$ is at least of first order in $\mu_{h}-\mu_{k}$; it is also of third order in $\mu$, because $\mu_{h}-\mu_{k}$ is of order $\mu^{2}$. So that for some constant $b_{3}$ it is $\left|D^{h, k}\right| \leq \bar{\varepsilon}^{3} b_{3} \gamma^{-\frac{1}{2}(k-h)}$, where the exponential decay in $k-h$ is due to the tree estimates of Sect. 5 and this can be used to get bounds on the coefficients $\bar{d}_{n}^{h, k}$ of the Taylor expansion of $\bar{D}^{h, k}$ in $\mu$ via Cauchy's theorem. It also follows that the coefficients $d_{n}^{h, k}$ depend only on $\delta_{m} \equiv c_{m}^{(h)}-c_{m}^{(k)}$ with $2 \leq m \leq n-1$ and are $\sum_{m=2}^{n-1} \delta_{m} \bar{d}_{n-m}^{h, k}$, so that

$$
\left|d_{n}^{h, k}\right| \leq d_{n} \gamma^{-\frac{k-h}{2}} \sup _{2 \leq m \leq n-1}\left|c_{m}^{(h)}-c_{m}^{(k)}\right|
$$

with $d_{n}$ than can be taken $\bar{\varepsilon}^{3} b_{3} \bar{\varepsilon}^{-n} n$.

Hence, if we define $d_{2}=0$, by (7.22) and (7.23), if $2 \leq n \leq r-1$ it is

$$
\left|c_{n}^{(h-1)}-c_{n}^{(h)}\right| \leq d_{n} \sum_{k=h+1}^{0} \gamma^{-\frac{k-h}{2}} \sup _{2 \leq m \leq n-1}\left|c_{m}^{(h)}-c_{m}^{(k)}\right|+O\left(\gamma^{h}\right)
$$

which easily implies that, if $n \leq r-1, c_{n} \equiv \lim _{h \rightarrow-\infty} c_{n}^{(h)}$ does exist and

$$
\left|c_{n}^{(h)}-c_{n}\right| \leq \bar{b} \gamma^{\theta h}
$$

for some constant $\bar{b}$, depending on $r$, and $0<\theta<1 / 2$. In fact, (7.25) is trivial for $n=2$; for $n>2$ it can be proved by induction, noting that $\left|c_{n}^{(h)}-c_{n}^{(k)}\right|$ does not appear in the r.h.s. of (7.24).

Finally, we have

$$
c_{r}^{(h-1)}=c_{r}^{(h)}+b_{r}+\sum_{k=h+1}^{0} d_{r}^{h, k}+O\left(\gamma^{h}\right),
$$

which would imply that $\left\{c_{r}^{(h)}\right\}_{h \leq 0}$ is a diverging sequence, in contradiction with the remark following (7.21), if the hypothesis (7.20) were verified; this easily follows by 
noting that, by (7.23) and (7.25), $\sum_{k=h+1}^{0} d_{r}^{h, k}$ is small of order $\gamma^{\theta h}$. Hence (7.6) is
proved.

Remark The idea behind the above argument is simply the following. The recursion relation is essentially local or with short memory: i.e. (7.2) is essentially a memoryless dynamical system because (7.23) shows that the memory, i.e. the number of scales $h^{\prime}$ above $h$ at which one must know $\mu_{h^{\prime}}$ in order to compute $\mu_{h-1}$ is essentially finite [by the exponential decay factor in (7.23)]. On the other hand a dynamical system without memory of the form $\mu_{h-1}=\mu_{h}+B\left(\mu_{h}\right)$ with $B$ analytic and vanishing at least to second order cannot have trajectories bounded by a constant $\tilde{\varepsilon}$ for all small enough initial data unless $B \equiv 0$.

We can now come back to our model; from now on $\mu_{h}$ will again denote the corresponding running couplings.

We note that, by (7.5), (7.6), (7.18), and (7.19):

$$
\mathscr{B}_{\mu}^{h}\left(\mu_{h}, \mu_{h+1}, \ldots, \mu_{0} ; \nu_{h}\right)=\sum_{k=h+1}^{0} D^{h, k}\left(\mu_{h}, \mu_{h+1}, \ldots, \mu_{0}\right)+O\left(\gamma^{h}\right) .
$$

Furthermore, the analysis of Sect. 5 implies that, if $\left|\mu_{k}\right| \leq \tilde{\varepsilon} \leq \varepsilon, k \geq h$, and $\tilde{\varepsilon}$ is small enough:

$$
\left|D^{h^{\prime}, k}\left(\mu_{h^{\prime}}, \mu_{h^{\prime}+1}, \ldots, \mu_{0}\right)\right| \leq b \tilde{\varepsilon} \gamma^{-\frac{k-h^{\prime}}{2}}\left|\mu_{k}-\mu_{h^{\prime}}\right|, \quad h^{\prime} \geq h,
$$

which implies that, for all $h^{\prime} \geq h$ :

$$
\left|\mu_{h^{\prime}-1}-\mu_{h^{\prime}}\right| \leq b \tilde{\varepsilon} \sum_{k=h^{\prime}+1}^{0} \gamma^{-\frac{k-h^{\prime}}{2}}\left|\mu_{k}-\mu_{h^{\prime}}\right|+O\left(\gamma^{h^{\prime}}\right) \text {. }
$$

By induction on $h^{\prime}$, it is easy to prove that $\left|\mu_{h^{\prime}-1}-\mu_{h^{\prime}}\right| \leq \tilde{b} \gamma^{\theta h^{\prime}}$, for any positive $\theta$ smaller than $1 / 2$ and a suitably chosen $\tilde{b}$, independent of $h^{\prime} \geq h$.

Hence it follows that, if $|\mu| \leq \bar{\varepsilon}$, with $\bar{\varepsilon}$ small enough, the sequence $\mu_{h}, h \leq 0$, is well defined and:

$$
\mu_{-\infty}=\lim _{h \rightarrow-\infty} \mu_{h}
$$

does exist as an analytic function of $\mu$, for $|\mu| \leq \bar{\varepsilon}$, if $\nu$ is suitably chosen (as an analytic function of $\mu$ ). Furthermore we can choose $\alpha$ (as a holomorphic function of $\lambda$ near $\lambda=0$ ), so that $\delta_{-\infty}=0$, if we want to impose that the Fourier transform of the pair Schwinger function behaves as $\left[k_{0}^{2}+e(\vec{k})^{2}\right]^{2 \eta}\left[-i k_{0}+e(\vec{k})\right]^{-1}$ near the Fermi surface (see $[B G, B G M]$ ). Hence our theory of the one dimensional spinless Fermi systems is complete, and it can be summarized in the theorem of Sect. 1.

\section{Appendix 1: Bounds on the Free Propagators}

In this appendix we want to prove the bounds (4.5), (5.27), and (6.17) on the single scale quasi-particle covariances.

We consider first $g_{\omega}^{(h)}(x)$; if $x=(t, \vec{x})$, we have:

$$
g_{\omega}^{(h)}(t, \vec{x})=g_{+1}^{(h)}(t, \omega \vec{x}),
$$


hence it is sufficient to consider the case $\omega=+1$. We write:

$$
g_{+1}^{(h)}(x)=\gamma^{h} \int_{1}^{\gamma^{2}} d \alpha \bar{g}_{h}(\alpha, \xi)
$$

where $\xi=\gamma^{h} x$ and

$$
\bar{g}_{h}(\alpha, \xi)=\int \frac{d k}{(2 \pi)^{2}} e^{-i k \xi-\alpha b(k)}\left(i k_{0}+\beta \bar{e}(\vec{k})\right) \chi\left(\vec{k}+\gamma^{-h} p_{F}\right)
$$

where $b(k)=\left(k_{0}^{2}+\beta^{2} \bar{e}(\vec{k})^{2}\right) p_{0}^{-1}$,

$$
\bar{e}(\vec{k})=\vec{k}\left(1+\vec{k} \frac{\gamma^{h}}{2 p_{F}}\right) .
$$

The $k_{0}$ integration can be explicitly performed and we get, if $\xi=\left(\xi_{0}, \vec{\xi}\right)$,

$$
\begin{aligned}
\bar{g}_{h}(\alpha, \xi)= & \frac{e^{-\frac{p_{0}^{2} \xi_{0}^{2}}{4 \alpha}}}{4 \pi^{3 / 2} \sqrt{\alpha}} \int d \vec{k} e^{-i \vec{k} \vec{\xi}-\alpha \beta^{2} p_{0}^{-2} \vec{k}^{2}(1+\varepsilon \vec{k})^{2}} \\
& \times\left[\frac{\xi_{0}}{2 \alpha}+\beta \vec{k}(1+\varepsilon \vec{k})\right] \chi\left(\vec{k}+\frac{1}{2 \varepsilon}\right),
\end{aligned}
$$

where $\varepsilon=\gamma^{h} /\left(2 p_{F}\right)$.

The integrand in the r.h.s. of (A1.5) is an analytic function of $\vec{k}$ in all the complex plane; hence we can shift the integration path in the imaginary direction, by putting $\vec{k}=p+i q$, with $q$ a fixed real number, having the same sign of $\vec{\xi}$. It is now very easy to show, by using the fact that $\alpha \geq 1$, that

$$
\left|\bar{g}_{h}(\alpha, \xi)\right| \leq c(q) e^{-|q||\xi|}
$$

where $c(q)$ is a suitable constant, independent of $h$.

The estimate (A1.6) and Eq. (A1.2) immediately imply the bound (4.5), for $m=0$. The bound on the derivatives of the covariance is obtained by a straightforward extension of the previous arguments.

Let us now come to the bound (5.27); by (5.9), we have to prove that a bound like (A1.6) is valid for the function

$$
\bar{r}_{h}(\alpha, \xi)=\int \frac{d k}{(2 \pi)^{2}} e^{-i k \quad \xi-\alpha b(k)}\left(i k_{0}+\beta \bar{e}(\vec{k})\right) \frac{z_{h}}{1+z_{h} e^{-b(k)}} \chi\left(\vec{k}+\gamma^{-h} p_{F}\right)
$$

for $1 \leq \alpha \leq 2$.

There are two differences with respect to the previous case. The first one is that we cannot explicitly perform the $k_{0}$ integration; we can solve this problem by shifting also the $k_{0}$ integration path. The second difference is that the integrand is not analytic in all the complex plane, as a function of $k_{0}$ and $\vec{k}$, because of the factor $\frac{z_{h}}{1+z_{h} e^{-b(k)}}$, which has an infinite number of poles. However, if $z_{h}$ is sufficiently small, for example $\left|z_{h}\right| \leq 1 / 2$, it is easy to see that we can find a strip around the real axis in both variables, so that the integrand is bounded and fast decreasing at infinity. Hence we can prove a bound like (A1.6), for $|q|$ small enough, say $|q|=\kappa$.

Finally, we shall prove the bound (6.17). 
Fig. 10.

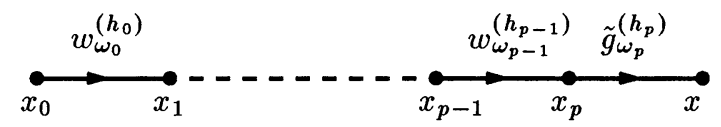

The recursive relation defining $Q_{h}$, in the first line of (6.14), can be easily solved; the solution can be graphically represented as a sum of chains of single scale propagators, separated by operators $z_{j} Z_{\jmath+1}\left[\partial_{t}+e\left(i \partial_{\vec{x}}\right]\right.$. If we insert the solution in (6.16), we get the following representation of $\tilde{g}_{Q, \omega}^{(h)}$ (see Fig. 10):

$$
\begin{aligned}
& \frac{1}{Z_{h}} \tilde{g}_{Q, \omega_{0}}^{(h)}\left(x-x_{0}\right)=\frac{1}{Z_{h}} \tilde{g}_{\omega_{0}}^{(h)}\left(x-x_{0}\right) \\
& \quad+\sum_{p=1}^{|h|} \sum_{h_{0}=h<h_{1}<} \sum_{\substack{h_{p} \leq 0 \\
k_{1} k_{p-1} \leq k_{\imath}<h_{\imath}}} \sum_{\omega_{1} \omega_{p}} \int d x_{1} \ldots d x_{p} \\
& \quad \times\left[\prod_{i=1}^{p} \frac{z_{k_{\imath}} Z_{k_{i}+1}}{Z_{h_{\imath-1}}} w_{\omega_{\imath}}^{\left(h_{\imath-1}\right)}\left(x_{i}-x_{i-1}\right)\right] \frac{1}{Z_{h_{p}}} \tilde{g}_{\omega_{p}}^{\left(h_{p}\right)}\left(x-x_{p}\right),
\end{aligned}
$$

where

$$
w_{\omega}^{(h)}(x)=e^{i p_{F} \omega \vec{x}} \int \frac{d k}{(2 \pi)^{2}} e^{-i k x}\left(-i k_{0}+e(\vec{k})\right) \tilde{g}^{(h)}(k) \chi\left(\omega \gamma^{-h} \vec{k}\right) .
$$

By the same arguments used above it is very easy to prove that

$$
\left|w_{\omega}^{(h)}(x)\right| \leq C \gamma^{2 h} e^{-\kappa \gamma^{h}|x|}
$$

Hence, if $\kappa^{\prime}<\kappa$ and $\left|z_{h}\right| \leq C \varepsilon^{2}$ for all $h$, we get the bound

$$
\begin{aligned}
e^{\kappa^{\prime} \gamma^{h}|x|}\left|\tilde{g}_{Q, \omega}^{(h)}(x)\right| & \leq C \gamma^{-h}+Z_{h} \sum_{p=1}^{|h|} \sum_{h_{0}=h<h_{1}<<h_{p} \leq 0} \sum_{\substack{k_{1} \\
h_{i-1} \leq k_{\imath}<h_{\imath}}}\left(C \varepsilon^{2}\right)^{p} \frac{\gamma^{-h_{p}}}{Z_{h_{p}}} \\
& \leq C \gamma^{-h}\left\{1+\sum_{p=1}^{\infty}\left(C \varepsilon^{2}\right)^{p}\left[\sum_{j=0}^{\infty} \gamma^{-j\left(1-C \varepsilon^{2}\right)}\right]^{2 p}\right\}
\end{aligned}
$$

which implies the bound (6.17) for $\varepsilon$ small enough.

\section{Appendix 2: The Gramm-Hadamard (and Related) Inequalities}

This appendix is mainly devoted to the proof of the bounds (3.43) and (5.57), involving the simple and truncated expectations of monomials in the field variables. We shall also study the "good" dependence on the volume of the simple expectation, see the remark after Lemma 1 in Sect. 7.

We shall first study the bounds involving the simple expectations. Then we consider a monomial in the fields of frequency $h$, which, in agreement with the notations of Sects. 3 and 5, we denote $\tilde{\psi}^{(h)}(P)$. In general $\tilde{\psi}^{(h)}(P)$ has the following structure:

$$
\tilde{\psi}^{(h)}(P)=\partial^{\left|q_{1}\right|} \psi_{x_{1}}^{+(h)} \ldots \partial^{\left|q_{m}\right|} \psi_{x_{m}}^{+(h)} \partial^{\left|q_{m+1}\right|} \psi_{x_{m+1}}^{-(h)} \ldots \partial^{\left|q_{2 m}\right|} \psi_{x_{2 m}}^{-(h)}
$$


where $2 m=|P|$ and

$$
\begin{aligned}
\partial^{\left|q_{j}\right|}=\partial_{t}^{q_{j}^{t}} \partial_{\vec{x}}^{q_{j}^{\vec{x}}}, & \text { if } h \leq 0, \\
\partial^{\left|q_{j}\right|}=\partial^{q_{j}^{t}} \Delta_{\vec{x}}^{q_{j}^{\vec{x}}}, & \text { if } h>0, \\
\left|q_{j}\right|=q_{j}^{t}+q_{j}^{\vec{x}} &
\end{aligned}
$$

with $q_{j}^{t}, q_{j}^{\vec{x}}$ non-negative integers. We shall also denote

$$
|q|=\left|q_{1}\right|+\ldots+\left|q_{2 m}\right|
$$

the total number of derivative operations present in the monomial $\tilde{\psi}^{(h)}(P)$.

Note that, when $h \leq 0$, the field variables depend also on the quasiparticle $\omega$ indices, but we have omitted them for the moment, to simplify the notation.

We will prove the following estimate:

$$
\left|\mathscr{E}_{h}\left[\tilde{\psi}^{(h)}(P)\right]\right| \leq C^{|P|} \gamma^{\frac{a(h)}{2}|P|} \gamma^{h|q|},
$$

where $a(h)=h$, if $h \leq 0$, and $a(h)=\frac{h}{2}$, if $h>0$. The bound (A2.4) immediately implies (3.43) and (5.57) in the case of the simple expectation $(s=1)$.

By the definition of simple expectation we can write:

$$
\mathscr{E}_{h}\left[\tilde{\psi}^{(h)}(P)\right] \leq \sum_{\mathscr{C}}(-1)^{\pi} \prod_{(\imath, \jmath) \in \mathscr{C}} \mathscr{E}_{h}\left[\partial^{\left|q_{m+i}\right|} \psi_{x_{m+2}}^{-(h)} \partial^{\left|q_{\jmath}\right|} \psi_{x_{j}}^{+(h)}\right],
$$

where the sum is over all the couplings, that is over all the possible ways to join each $\psi^{-}$variable with a $\psi^{+}$variable, and $(-1)^{\pi}$ is the parity of the permutations which bring next to each other the joined variables, with the $\psi^{-}$variable on the left.

It is an easy task to show that (A2.5) may be rewritten as a determinant, up to a sign:

$$
\mathscr{E}_{h}\left[\tilde{\psi}^{(h)}(P)\right]= \pm \operatorname{det} g^{(h)},
$$

where $g^{(h)}$ is the $m \times m$ matrix with elements [see (3.22) and (5.22)]:

$$
g_{i j}^{(h)}=\left\{\begin{array}{ll}
\partial^{\left|q_{m+\imath}\right|} \partial^{\left|q_{\jmath}\right|} C_{h}\left(x_{m+\imath}-x_{\jmath}\right) & \text { if } h>0 \\
\partial^{\left|q_{m+\imath}\right|} \partial^{\left|q_{\jmath}\right|} \delta_{\omega_{\imath} \omega_{\jmath}} \tilde{g}_{\omega_{\imath}}^{h}\left(x_{m+i}-x_{j}\right) & \text { if } h \leq 0
\end{array} .\right.
$$

In order to show (A2.4) we need a good bound of the determinant in (A2.6); we shall use the well known Gramm-Hadamard inequality. Let $M$ be a square matrix, with elements $M_{\alpha \beta}$, and suppose that $M_{\alpha \beta}$ can be written as

$$
M_{\alpha \beta}=\left(A_{\alpha}, B_{\beta}\right) \text {, }
$$

where $A_{\alpha}$ and $B_{\beta}$ are vectors in a Hilbert space with scalar product $(\cdot, \cdot)$. Then the following inequality is satisfied:

$$
|\operatorname{det} M| \leq \prod_{\alpha}\left\|A_{\alpha}\right\|\left\|B_{\alpha}\right\|
$$

where $\|\cdot\|$ is the norm induced by the scalar product.

Hence (A2.4) will be proved, if we show that, both in the ultraviolet and in the infrared case, the matrix $g^{(h)}$ can be written as in (A2.8), with:

$$
\left\|A_{i}\right\| \leq C \gamma^{a(h) / 2+h\left|q_{m+\imath}\right|}, \quad\left\|B_{j}\right\| \leq C \gamma^{a(h) / 2+h\left|q_{\jmath}\right|}
$$


Let us define

$$
g^{(h)}(x)=\left\{\begin{array}{ll}
C_{h}(x) & \text { if } h>0 \\
\tilde{g}_{\omega}^{(h)}(x) & \text { if } h \leq 0
\end{array},\right.
$$

and note that the Fourier transform $g^{(h)}(x)$ satisfies, for any $n \geq 0$, the following bound:

$$
\int|k|^{n}\left|\hat{g}^{(h)}(k)\right| d^{2} k \leq C_{n} \gamma^{a(h)+n} .
$$

This immediately follows, for $h>0$, from the definition (3.21), that is

$$
C_{h}(x)=f\left(\gamma^{h} x_{0}\right) h(\vec{x}) e^{\frac{x_{0} p_{F}^{2}}{2 m}}\left(\frac{m}{2 \pi x_{0}}\right)^{d / 2} e^{-\frac{m \vec{x}^{2}}{2 x_{0}}},
$$

and from the remark that the functions $f\left(x_{0}\right)$ and $h(\vec{x})$ were chosen as smooth functions. For $h \leq 0$, (A2.12) follows very easily from the expression for the Fourier transform of $\tilde{g}_{\omega}^{(h)}$, given in Appendix 1 .

Let us now observe that we can write

$$
\begin{aligned}
\partial^{\left|q_{m+\imath}\right|} \partial^{\left|q_{j}\right|} g^{(h)}\left(x_{m+i}-x_{j}\right)= & \int \frac{d k}{(2 \pi)^{2}} e^{-\imath k\left(x_{m+\imath}-x_{\jmath}\right)}(-i k)^{\left(\left|q_{m+\imath}\right|+\left|q_{\jmath}\right|\right)} \hat{g}^{(h)}(k) \\
= & \int d z \int \frac{d k}{(2 \pi)^{2}} e^{-i k\left(x_{m+\imath}-z\right)}(-i k)^{\left|q_{m+\imath}\right|} \hat{A}_{h}(k) \\
& \times \int \frac{d k}{(2 \pi)^{2}} e^{-i k^{\prime}\left(x_{\jmath}-z\right)}\left(-i k^{\prime}\right)^{\left|q_{\jmath}\right|} \hat{B}_{h}\left(k^{\prime}\right), \quad \text { (A2. }
\end{aligned}
$$

where $(-i k)^{|q|} \equiv\left(-i k_{0}\right)^{q^{t}}(-i \vec{k})^{q^{\vec{x}}}$ and

$$
\begin{aligned}
& \hat{A}_{h}(k)=\left(\left|\hat{g}^{(h)}(k)\right|^{2}\right)^{3 / 4} \hat{g}^{(h) *}(k)^{-1}, \\
& \hat{B}_{h}(k)=\left(\left|\hat{g}^{(h)}(k)\right|^{2}\right)^{1 / 4} .
\end{aligned}
$$

Hence, if $h>0$, we define:

$$
\begin{aligned}
& A_{i}^{(h)}(z)=\int \frac{d k}{(2 \pi)^{2}} e^{i k\left(x_{m+\imath}-z\right)}(+i k)^{\left|q_{m+\imath}\right|} \hat{A}_{h}^{*}(k), \\
& B_{j}^{(h)}(z)=\int \frac{d k}{(2 \pi)^{2}} e^{-\imath k\left(x_{\jmath}-z\right)}(-i k)^{\left|q_{\jmath}\right|} \mid \hat{B}_{h}(k),
\end{aligned}
$$

so that, by (A2.12), $A_{i}^{(h)}$ and $B_{j}^{(h)}$ are $L_{2}$ functions, satisfying the relations (A2.8) and (A2.10) with respect to the $L_{2}$ scalar product.

If $h \leq 0$, we have to take into account also the $\omega$ dependence. This is easily done by considering, in the tensor product of $L_{2}\left(\mathbf{R}^{2}\right)$ and $\mathbf{C}^{2}$ :

$$
\begin{aligned}
& A_{i}=A_{i}^{(h)}(z) \otimes S_{\omega_{\imath}}, \\
& B_{\jmath}=B_{j}^{(h)}(z) \otimes S_{\omega_{\jmath}},
\end{aligned}
$$

where $S_{\omega} \in \mathbf{C}^{2}$ is defined by

$$
S_{\omega}= \begin{cases}\left(\begin{array}{l}
1 \\
0
\end{array}\right) & \text { if } \omega=+1 \\
\left(\begin{array}{l}
0 \\
1
\end{array}\right) & \text { if } \omega=-1\end{cases}
$$


so that

$$
\left(S_{\omega_{\imath}}, S_{\omega_{\jmath}}\right)=\delta_{\omega_{\imath} \omega_{\jmath}}, \quad\left\|S_{\omega_{i}}\right\|=\left\|S_{\omega_{\jmath}}\right\|=1 .
$$

This concludes our discussion for the simple expectations.

The bounds on the truncated expectations are obtained by using for them the well known expansion in terms of interpolating parameters (see, for example [B]), as in [Le].

It turns out that the sum of the connected graphs can be written in the following way:

$$
\begin{aligned}
\mathscr{E}_{h}^{T}\left(\tilde{\psi}\left(P_{1}\right), \ldots, \tilde{\psi}\left(P_{k}\right)\right)= & \int \prod_{j=1}^{k} \prod_{i=1}^{p_{j}} d \eta_{\jmath, i} \prod_{i=1}^{q_{\jmath}} d \bar{\eta}_{j, i} \\
& \times \sum_{\tilde{T}} \prod_{\left(j, j^{\prime}\right) \in \tilde{T}}\left(V_{j, j^{\prime}}+V_{j^{\prime}, j}\right) \int d P_{\tilde{T}}(s) e^{-V(s)},
\end{aligned}
$$

where

1) $\eta_{j, i}$ and $\bar{\eta}_{j, i}$ are Grassmannian variables, each associated with the $i^{\text {th }}$ field of the $j^{\text {th }}$ monomial (cluster) of fields appearing in (A2.20). The fields on scale $h$ will be denoted from now on with $\psi_{x_{j, i}}^{\sigma_{j, i}}$ and $g^{(h)}\left(x_{j^{\prime}, i^{\prime}}-x_{j, i}\right)$ will denote the corresponding covariance; $p_{\jmath}\left(q_{j}\right)$ are the number of $\psi^{+}\left(\psi^{-}\right)$fields in the $j^{\text {th }}$ cluster and $\sum_{j} p_{j}=\sum_{\jmath} q_{\jmath}=n$. We are assuming for the sake of simplicity that no derivative fields are present.

2) $\sum_{\tilde{T}}$ is the sum over all the tree graphs between the clusters thought of as points.

3) $V_{j, j^{\prime}}=\sum_{i^{\prime}=1}^{q_{\jmath^{\prime}}} \sum_{i=1}^{q_{j}} \bar{\eta}_{j^{\prime}, i^{\prime}} g^{(h)}\left(x_{j^{\prime}, i^{\prime}}-x_{\jmath, 2}\right) \eta_{j, i}$ and $V(s)=\sum_{j=1}^{k} V_{j, \jmath}+\sum_{j \neq \jmath^{\prime}} S_{j j^{\prime}} V_{j, \jmath^{\prime}}$.

4) $S_{j j^{\prime}}$ is a product of interpolating parameters $s_{n}, n=1, \ldots, k-1$, valued in $[0,1]$, and the clusters can be ordered in such a way that $S_{j j^{\prime}}=\prod_{n=j}^{j^{\prime}-1} s_{n}\left(j^{\prime}>j\right)$.

5) $d P_{\tilde{T}}(s)$ is a normalized measure, $\int d P_{\tilde{T}}(s)=1$, which depends on the interpolating parameters $s_{n}$ and on $\tilde{T}$.

It is easy to extract from (A2.20) the exponential factor appearing in (3.43) and (5.57). Let us in fact develop, for a fixed tree graph $\tilde{T}$, the product $\prod_{\left(\jmath, \jmath^{\prime}\right)}\left(V_{\jmath, \jmath^{\prime}}+V_{j^{\prime}, j}\right)$; we get:

$$
\begin{aligned}
& \prod_{\left(j, j^{\prime}\right)}\left(V_{j, j^{\prime}}+V_{j^{\prime}, j}\right)=\sum_{i_{1}, \iota_{k-1}} \sum_{i_{1}^{\prime},,_{i}^{\prime}{ }_{k-1}} \bar{\eta}_{j_{1}^{\prime}, i_{1}^{\prime}} \eta_{j_{1}, i_{1}} \ldots \bar{\eta}_{j_{k-1}^{\prime}, i_{k-1}^{\prime}} \eta_{\jmath_{k-1}, i_{k-1}} \\
& \quad \times g^{(h)}\left(x_{j_{1}^{\prime}, i_{1}^{\prime}}-x_{\jmath_{1}, \imath_{1}}\right) \ldots g^{(h)}\left(x_{\jmath_{k-1}^{\prime}, i_{k-1}^{\prime}}-x_{\jmath_{k-1}, i_{k-1}}\right) .
\end{aligned}
$$

Recalling the definitions of Sects. 3 and 5 of anchored tree graph, it is now obvious that once $\tilde{T}$ and the sets $i_{1}, \ldots, i_{k-1}, i_{1}^{\prime}, \ldots, i_{k-1}^{\prime}$ are fixed, an anchored tree graph $T$ is also uniquely chosen. We recall that $T$ is a set of $k-1$ difference vectors $x_{j_{1}^{\prime}, i_{1}^{\prime}}-x_{j_{1}, i_{1}}, \ldots, x_{j_{k-1}^{\prime}, i_{k-1}^{\prime}}-x_{j_{k-1}, i_{k-1}}$ which realize the connection between the $k$ clusters of fields $\psi\left(P_{1}\right), \ldots, \psi\left(P_{k}\right)$, see remarks after (3.43) and (5.57). Thus we can rewrite

$$
\sum_{\tilde{T}} \sum_{i_{1}, \imath_{k-1}} \sum_{i_{1}^{\prime}, \imath_{k-1}^{\prime}}=\sum_{T} .
$$


Now, using the bounds (3.22) and (5.27), we can also write

$$
\begin{aligned}
& \left|g^{(h)}\left(x_{j_{1}^{\prime}, i_{1}^{\prime}}-x_{j_{1}, i_{1}}\right) \ldots g^{(h)}\left(x_{\jmath_{k-1}^{\prime}, \iota_{k-1}^{\prime}}-x_{j_{k-1}, i_{k-1}}\right)\right| \\
& \quad \leq C^{k-1} \gamma^{a(h)(k-1)} e^{-\kappa d_{T}^{h}\left(P_{1}, \quad, P_{s}\right)},
\end{aligned}
$$

where $d_{T}^{h}\left(P_{1}, \ldots, P_{s}\right)$ is defined as in (3.43) or as in (5.57). Hence we can bound (A2.20) as

$$
\begin{aligned}
& \left|\mathscr{E}_{h}^{T}\left(\tilde{\psi}\left(P_{1}\right), \ldots, \tilde{\psi}\left(P_{k}\right)\right)\right| \leq C^{k-1} \gamma^{a(h)(k-1)} \sum_{T} e^{-\kappa d_{T}^{h}\left(P_{1}, \quad, P_{s}\right)} \\
& \quad \times\left|\int \prod_{j=1}^{k} \prod_{i=1}^{p_{j}} d \eta_{j, i} \prod_{\imath=1}^{q_{j}} d \bar{\eta}_{\jmath, \imath} \eta^{T} \bar{\eta}^{T} \int d P_{T}(s) e^{-V(s)}\right|,
\end{aligned}
$$

where $\eta^{T} \bar{\eta}^{T}=\eta_{\jmath_{1}, i_{1}} \bar{\eta}_{j_{1}^{\prime}, i_{1}^{\prime}} \ldots \eta_{j_{k-1}, i_{k-1}} \bar{\eta}_{\jmath_{k-1}^{\prime}, \imath_{k-1}^{\prime}}$.

It is now a standard task to prove, using the properties of the Grassmannian variables, that, for a fixed anchored tree graph $T$, the integration over the variables $\eta_{j, i}, \bar{\eta}_{\jmath, \imath}$ gives

$$
\int \prod_{\jmath=1}^{k} \prod_{i=1}^{p_{\jmath}} d \eta_{j, i} \prod_{i=1}^{q_{\jmath}} d \bar{\eta}_{\jmath, \imath} \eta^{T} \bar{\eta}^{T} e^{-V(s)}=\operatorname{det} G^{T}(s)
$$

where $G^{T}(s)$ is a $(n-k+1) \times(n-k+1)$ matrix whose elements are $G_{j i j^{\prime} i^{\prime}}^{T}=$ $S_{j j^{\prime}} g^{(h)}\left(x_{j^{\prime}, i^{\prime}}-x_{j, i}\right)$ with $x_{j^{\prime}, i^{\prime}}-x_{j, i}$ not belonging to the anchored tree graph $T$. Such a determinant can be bounded again using Gramm-Hadamard inequality. In fact $G_{j i j^{\prime} i^{\prime}}^{T}$ can be rewritten as a scalar product of two vectors, as in (A2.8), performing the tensor product between the $A_{j i}$ and $B_{\imath^{\prime} \jmath^{\prime}}$ defined as in (A2.16) (taking care of the indices) and the vector $e_{j}$ defined as follows [Le]. Let $v_{i} \in \mathbf{C}^{k}$ be the unit vector $\left(v_{i}\right)_{j}=\delta_{i j}$; then the $e_{j}$ are defined inductively by

$$
e_{1}=v_{1}, \quad e_{j}=s_{j-1} e_{\jmath-1}+\left(1-s_{j-1}^{2}\right)^{1 / 2} v_{j}, \quad j=2, \ldots, k-1,
$$

which implies that

$$
\left\|e_{j}\right\|=1, \quad\left(e_{j}, e_{j^{\prime}}\right)=s_{j} s_{j+1} \ldots s_{j^{\prime}-1}=S_{j j^{\prime}},
$$

where $(, \cdot)$ denotes the usual scalar product in $\mathbf{C}^{k}$.

Hence, writing $G_{j i j^{\prime} i^{\prime}}^{T}=\left(e_{j} \otimes A_{j i}, e_{j^{\prime}} \otimes B_{j^{\prime} i^{\prime}}\right)$ and performing the same steps as before we obtain the following bound:

$$
\left|\operatorname{det} G^{T}(s)\right| \leq \gamma^{\frac{a(h)}{2} \sum_{j=1}^{k}\left|P_{\jmath}\right|-a(h)(k-1)} C^{\frac{1}{2} \sum_{\jmath=1}^{k}\left|P_{\jmath}\right|-(k-1)} .
$$

Inserting now (A2.28) in (A2.24) and taking into account item 5) above, we obtain the bounds (3.43) and (5.57) for the case that no derivative is acting on the fields. The generalization to the case in which also derivative fields are allowed is trivial and we left it to the reader.

Finally we want to show the result claimed in Lemma 1 of Sect. 7; i.e. we want to compare the finite and infinite volume expectations of a generic monomial $\tilde{\psi}^{(h)}(P)$. 
We can obviously define in the finite volume the vectors $A_{\imath}^{(h, L)}, B_{j}^{(h, L)}$ such that

$$
\left(A_{\imath}^{(h, L)}, B_{j}^{(h, L)}\right)=g_{i j}^{(h, L)} .
$$

This is done in a way totally analogous to (A2.16), with the integral replaced by a sum. The result that now we want to prove is therefore the following:

$$
\left|\operatorname{det}\left(A_{i}^{(h)}, B_{\jmath}^{(h)}\right)-\operatorname{det}\left(A_{i}^{(h, L)}, B_{\jmath}^{(h, L)}\right)\right| \leq \frac{C^{|P|}}{L} \gamma^{\frac{a(h)}{2}|P|} \gamma^{h|q|} .
$$

This is easily achieved using the obvious property:

$$
\left\|A_{\imath}^{(h)}-A_{i}^{(h, L)}\right\| \leq \frac{C}{L} \gamma^{\frac{a(h)}{2}+h\left|q_{m+\imath}\right|}, \quad\left\|B_{j}^{(h)}-B_{j}^{(h, L)}\right\| \leq \frac{C}{L} \gamma^{\frac{a(h)}{2}+h\left|q_{j}\right|}
$$

and the well known relation:

$$
\operatorname{det}\left(M+M^{\prime}\right)=\sum_{m} \operatorname{det}_{m}(M) \operatorname{det}_{m^{c}}\left(M^{\prime}\right)+\operatorname{det} M+\operatorname{det} M^{\prime},
$$

where $\sum_{m}$ is the sum over the (non-void) minors of the matrix $M+M^{\prime}$, and $\operatorname{det}_{m}(M)$ $\left(\operatorname{det}_{m^{c}}\left(M^{\prime}\right)\right.$ ) is the determinant of the minor $m$ (of the complementary $m^{c}$ of $m$ ) of the corresponding matrix.

In fact, let us write:

$$
\begin{aligned}
\operatorname{det}\left(A_{i}^{(h)}, B_{\jmath}^{(h)}\right) & =\operatorname{det}\left(A_{\imath}^{(h)}+A_{i}^{(h, L)}-A_{i}^{(h, L)}, B_{\jmath}^{(h, L)}\right) \\
& =\operatorname{det}\left(\left(A_{\imath}^{(h)}-A_{\imath}^{(h, L)}, B_{j}^{(h, L)}\right)+\left(A_{i}^{(h, L)}, B_{j}^{(h)}\right)\right) .
\end{aligned}
$$

Using now (A2.32) we have:

$$
\begin{aligned}
\operatorname{det}\left(A_{i}^{(h)}, B_{\jmath}^{(h)}\right)= & \operatorname{det}\left(A_{\imath}^{(h, L)}, B_{\jmath}^{(h)}\right) \\
& +\sum_{m} \operatorname{det}_{m}\left(A_{i}^{(h)}-A_{i}^{(h, L)}, B_{j}^{(h)}\right) \operatorname{det}_{m^{c}}\left(A_{i}^{(h, L)}, B_{j}^{(h)}\right) \\
& +\operatorname{det}\left(A_{i}^{(h)}-A_{\imath}^{(h, L)}, B_{\jmath}^{(h)}\right)
\end{aligned}
$$

Using (A2.31) and the Gramm-Hadamard inequality it is now obvious that the second and third addend in the r.h.s. of (A2.34) can be bounded by $\frac{C_{1}^{|P|}}{L} \gamma^{\frac{a(h)}{2}|P|} \gamma^{h|q|}$ (note that the total number of non-void minors is $4^{|P| / 2}-2$ ). Repeating the same argument for $B_{j}^{(h)}$ we obtain (A2.30).

\section{Appendix 3. The Bound (5.60)}

In this section we want to prove the bound (5.60). We can write:

$$
J\left(\tau,\left\{h_{v}\right\}, P_{v_{0}}, \underline{x}_{v_{0}}\right)=\sum_{T}\left(\prod_{v \text { not e p }} \frac{1}{s_{v} !} \int d \underline{r}^{\left(P_{v}\right)}\right) J_{T}(\underline{x}, \underline{r}),
$$

where

$$
J_{T}(\underline{x}, \underline{r})=\left(\prod_{l \in T} e^{-\kappa \gamma^{h_{l}}\left|\xi_{l}-\eta_{l}\right|}\right)\left(\prod_{v}\left(\xi_{v}-\xi_{v}^{\prime}\right)^{\bar{z}_{v}}\right) .
$$


Here $\underline{r}$ is the set of all interpolation parameters and $T$ is a set of lines obtained by choosing one of the anchored trees $T_{v}$ in each non-trivial vertex. Moreover, if $l \in T$, we denote $h_{l}$ the corresponding frequency index and $\xi_{l}, \eta_{l}$ its endpoints; $h_{l}$ is the frequency of the contraction between the two field variables, emerging from the space vertices $\xi_{l}$ and $\eta_{l}$, which gave rise to the factor $e^{-\kappa \gamma^{h}}\left|\xi_{l}-\eta_{l}\right|$ (see Appendix 2).

Note that $\xi_{l}$ and $\eta_{l}$

a) either coincide with one of the integration variables $\underline{x}$, and in this case we shall say that they are simple space vertices;

b) or are convex combinations of the integration variables through the interpolation parameters, and we shall say that they are interpolated space vertices.

Note also that $T$ is not in general a tree, if some space vertex is an interpolated one. However, we can uniquely associate to $T$ a tree $\tilde{T}$ connecting the set $\underline{x}$ of the integration variables, by substituting $\xi_{l}$ and $\eta_{l}$ with the space vertices $x_{l}$ and $y_{l}$ (which can coincide with them), from which the corresponding field variables emerge before the application of the $\mathscr{R}$ operations [see Sect. 5, item 5 before (5.31)]. There is of course a one-to-one correspondence between the lines of $T$ and $\tilde{T}$.

Given a non-trivial vertex $v \in \tau$, we shall denote $\tilde{S}_{v}$ the subset of $\tilde{T}$, connecting the points in $\underline{x}_{v}$ (recall that $\underline{x}_{v}$ is the set of integration variables associated to the vertex $v$ ) and $S_{v}$ the corresponding subset of $T$; of course:

$$
S_{v}=\bigcup_{\mathrm{nt}} T_{\bar{v} \geq v}
$$

Finally, we shall say that a line in $T$ is a simple line if it connects two simple space vertices, an interpolated line if one of its endpoints is an interpolated space vertex; note that, if the line $l \in T$ is a simple line, then it is also true that $l \in \tilde{T}$.

The main point of this appendix is the proof that

$$
\left|J_{T}(\underline{x}, \underline{r})\right| \leq\left(\prod_{l \in \tilde{T}} e^{-\bar{\kappa} \gamma h_{l} l\left|x_{l}-y_{l}\right|}\right)\left(\prod_{v} C \gamma^{-h_{v} \bar{z}_{v}}\right),
$$

where $C$ is a suitable constant and

$$
\bar{\kappa}<\frac{\kappa}{2}\left(1-\frac{1}{\gamma}\right)
$$

As in Sect. 5, we shall suppose, for simplicity, that only local terms are associated to the endpoints of $\tau$.

We first bound the factors $\left(\xi_{v}-\xi_{v}^{\prime}\right)^{\bar{z}_{v}}$; recall that $\bar{z}_{v}$ is a positive integer $\leq 2$ and that $\left(\xi_{v}-\xi_{v}^{\prime}\right)^{\bar{z}_{v}}$ denotes the tensor of rank two, if $\bar{z}_{v}=2$. We can write:

$$
\xi_{v}=\sum_{i=1}^{r} \lambda_{i} x_{i}, \quad \xi_{v}^{\prime}=\sum_{j=1}^{s} \mu_{j} y_{j}, \quad x_{i}, y_{j} \in \underline{x}_{v}
$$

where $\lambda_{i}$ and $\mu_{j}$ are interpolation parameters, hence they are positive and $\sum_{i=1}^{r} \lambda_{i}=$ $\sum_{j=1}^{s} \mu_{j}=1$.

We have, for any $\varepsilon>0$,

$$
\left|\xi_{v}-\xi_{v}^{\prime}\right| \leq \sup _{i, j}\left|x_{i}-y_{j}\right| \leq C_{\varepsilon} \gamma^{-h_{v}} e^{\frac{\varepsilon}{2} \gamma^{h_{v}}} \sum_{l \in \tilde{S}_{v}}\left|x_{l}-y_{l}\right|
$$


where $C_{\varepsilon}$ is a suitable constant and $\tilde{S}_{v}$ is defined before (A3.3). Since $\bar{z}_{v} \leq 2$, (A3.7) implies that

$$
\left|\xi_{v}-\xi_{v}^{\prime}\right|^{\bar{z}_{v}} \leq C_{\varepsilon}^{2} \gamma^{-h_{v} \bar{z}_{v}} e^{\varepsilon \gamma^{h_{v}}} \sum_{l \in \tilde{S}_{v}}\left|x_{l}-y_{l}\right|
$$

We observe now that, given any line $l \in \tilde{T}$, we can associate to it all the factors $e^{\varepsilon \gamma^{h} l\left|x_{l}-y_{l}\right|}$ coming from the r.h.s. of (A3.8), for each non-trivial vertex containing that line; the product of these factors can be bounded by $e^{2 \varepsilon\left|x_{l}-y_{l}\right|} \sum_{h \leq h_{l}} \gamma^{h}$ (the factor 2 in the exponent comes from the observation that, in each line of $\tau$ connecting two non-trivial vertices, at most two trivial vertices can array a factor $\left|\xi_{v}-\xi_{v}^{\prime}\right| \bar{z}_{v}$ with $\bar{z}_{v}>0$ ). Hence we have:

$$
\prod_{v}\left|\xi_{v}-\xi_{v}^{\prime}\right|^{\bar{z}_{v}} \leq\left(\prod_{v} C_{\varepsilon}^{2} \gamma^{-h_{v} \bar{z}_{v}}\right)\left(\prod_{l \in \tilde{T}} e^{\frac{2 \varepsilon}{1-1 / \gamma} \gamma^{h_{l}}\left|x_{l}-y_{l}\right|}\right)
$$

In order to complete the proof of (A3.4), we have to bound the first factor in the r.h.s. of (A3.2). Let us define $\kappa^{\prime}$ so that

$$
\kappa \gamma^{h}=2 \kappa^{\prime} \sum_{p=0}^{\infty} \gamma^{h-p}
$$

that is

$$
\kappa^{\prime}=\frac{\kappa}{2}\left(1-\frac{1}{\gamma}\right)
$$

Hence we have, for any $l \in T$ :

$$
e^{-\kappa \gamma^{h} l\left|\xi_{l}-\eta_{l}\right|}=\prod_{h \leq h_{l}}\left[e^{-\kappa^{\prime} \gamma^{h}\left|\xi_{l}-\eta_{l}\right|}\right]^{2}
$$

If the line $l \in T$ is a simple line, we associate to it a factor $e^{-\kappa^{\prime} \gamma^{h} l\left|\xi_{l}-\eta_{l}\right|}$, taken from the r.h.s. of (A3.12), whose remaining part will be used as explained below. Note that all the lines associated to the higher non-trivial vertices in $\tau$, different from the endpoints, are simple lines.

Let us now suppose that the line $l \in T$ is an interpolated line, but $y_{l}$ is a simple space vertex. We can write $\xi_{l}=\sum_{i=1}^{r} \lambda_{i} x_{i}$, with $x_{\imath}$ simple space vertices associated to some non-trivial vertex $v$ of $\tau$, having frequency index $h_{v}>h_{l}$; the set $\left\{x_{1}, \ldots, x_{r}\right\}$ has to contain the special space vertex $x_{l}$ [see the remark before (A3.4)]. We have:

$$
\begin{aligned}
\left|x_{l}-y_{l}\right| & \leq\left|x_{l}-\xi_{l}\right|+\left|\xi_{l}-y_{l}\right| \leq \sum_{\imath=1}^{r} \lambda_{i}\left|x_{\imath}-x_{l}\right|+\left|\xi_{l}-y_{l}\right| \\
& \leq\left|\bar{x}-x_{l}\right|+\left|\xi_{l}-y_{l}\right|
\end{aligned}
$$

where $\bar{x}$ is defined so that $\left|\bar{x}-x_{l}\right|=\sup _{i}\left|x_{i}-x_{l}\right|$. 
Fig. 11.

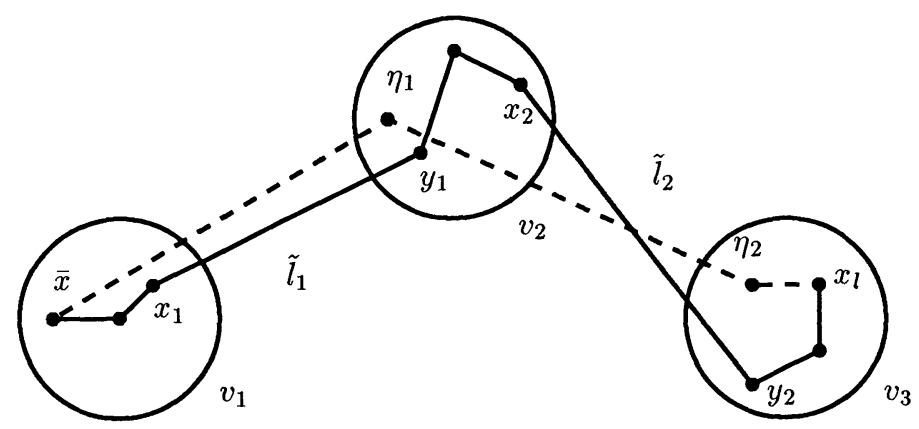

In the tree $\tilde{S}_{v}$ we can find a unique path $\mathscr{C}$ connecting $\bar{x}$ to $x_{l}$. We shall distinguish two cases.

a) $\mathscr{C}$ is made by lines belonging also to $T$. In this case, for any $\bar{l} \in \mathscr{C}$, since $h_{\bar{l}}>h_{l}$, we can extract from the r.h.s. of (A3.12) a factor $e^{-\kappa^{\prime} \gamma^{h} l\left|x_{\bar{l}}-y_{\bar{l}}\right|}$; then we associate to $l$ all these factors, together with the factor $e^{-\kappa^{\prime} \gamma^{h} l\left|\xi_{l}-y_{l}\right|}$ coming again from (A3.12), applied to the line $l$ itself. Hence, by using (A3.13) and the trivial inequality

$$
\left|\bar{x}-x_{l}\right| \leq \sum_{\bar{l} \in \mathcal{Y}_{l}}\left|x_{\bar{l}}-y_{\bar{l}}\right|
$$

we can bound the overall factor associated to the line $l$ by $e^{-\kappa^{\prime} \gamma^{h} l\left|x_{l}-y_{l}\right|}$, as in the case of the simple lines.

b) At least one line of $\mathscr{C}$ does not belong to $T$. In this case, the inequality (A3.14) is not useful; however, if we can associate to $\mathscr{C}$ a subset $T_{l}$ of $S_{v}$, such that

$$
\left|\bar{x}-x_{l}\right| \leq \sum_{\bar{l} \in T_{l}}\left|\xi_{\bar{l}}-\eta_{\bar{l}}\right|
$$

the argument of item a) can be immediately generalized. We shall now prove that this is in fact possible.

Let $\bar{v}$ be the higher non-trivial vertex containing $\mathscr{C}$ and let $v_{1}, \ldots, v_{s}, 2 \leq s \leq s_{\bar{v}}$, be the non-trivial vertices or endpoints following $\bar{v}$ in $\tau$, which are intersected by $\mathscr{C}$, that is such that at least one line of $\mathscr{C}$ has an endpoint belonging to $x_{v_{2}}$, for any $i=1, \ldots, s$; at least one of these vertices has to be different from an endpoint of $\tau$, otherwise we would be in the situation of item a), since all the lines associated to the higher non-trivial vertices of $\tau$ are simple lines. The $v_{i}$ are ordered so that, if we fix a positive direction in the path $\mathscr{C}$, going from $\bar{x}$ to $x_{l}, v_{i}$ is crossed by $\mathscr{C}$ before $v_{j}$, if $i<j$ (see Fig. 11).

Let $\tilde{l}_{\imath}, i=1, \ldots, s-1$, be the line of $\mathscr{C}$ going from $v_{i}$ to $v_{\imath+1}$ and let $x_{i} \in \underline{x}_{v_{\imath}}$, $y_{i} \in \underline{x}_{v_{\imath+1}}$ be the endpoints of $\tilde{l}_{\imath}$; we shall denote by $\xi_{i}, \eta_{i}$ the corresponding endpoints of the line $\bar{l}_{i} \in T$, which corresponds to $\tilde{l}_{i}$. We consider now the path $\mathscr{C}^{(1)}$, connecting $\bar{x}$ to $x_{l}$, obtained by taking the $s-1$ lines $l_{\imath}$ with endpoints $\eta_{i-1}$ and $\eta_{i}$ (we define $\left.\eta_{0}=\bar{x}\right)$ and the line with endpoints $\xi_{s} \equiv x_{l}$ and $\eta_{s-1}$, see Fig. 11 . 
We have

$$
\left|\bar{x}-x_{l}\right| \leq \sum_{i=1}^{s-1}\left|\eta_{i}-\eta_{i-1}\right|+\left|x_{l}-\eta_{s-1}\right| \leq \sum_{i=1}^{s-1}\left(\left|\eta_{\imath}-\xi_{i}\right|\right)+\sum_{i=1}^{s}\left(\left|\xi_{i}-\eta_{i-1}\right|\right) \text {. }
$$

Moreover, $\forall i=1, \ldots, s, \xi_{i}$ and $\eta_{\imath-1}$ either coincide or are convex combination of space vertices contained in the same cluster $\underline{x}_{v_{\imath}}$, so that:

$$
\left|\xi_{\imath}-\eta_{i-1}\right| \leq \sup _{x, y \in \underline{x}_{v_{\imath}}}|x-y|=\left|\bar{x}_{i}-\bar{y}_{\imath}\right| .
$$

We can now define inductively the construction of the set $T_{l}$, satisfying (A3.15). We first put in $T_{l}$ the lines $l_{i}, i=1, ., s-1$, then we consider all vertices $v_{i}$, such that $\xi_{i} \neq \eta_{i-1}$, and we look for new sets of lines, contained in $S_{v_{\imath}}$, whose total length is larger than $\left|\bar{x}_{\imath}-\bar{y}_{\imath}\right|$. This can be done by iterating the previous procedure and the overall construction will end when all the vertices involved are endpoints of $\tau$ or non-trivial vertices containing only simple lines, for which the construction of item a) can be applied; this will happen after a finite number of steps, by the remark above about the higher non-trivial vertices. The bounds (A3.16) and (A3.17) immediately imply that the set $T_{l}$ satisfy (A3.15).

The previous construction can be extended without any difficulty to the case of a line $l$, whose endpoints are both interpolated space vertices. In fact the two corresponding sets of interpolated space vertices have to belong to two disjoint vertices of $\tau$.

To complete the proof of (A3.4), it is sufficient to observe that, if we apply previous construction to all the lines of $T$, each line contributes a factor $e^{-\kappa^{\prime} \gamma^{h}\left|\xi_{l}-\eta_{l}\right|}$, for each $h \leq h_{l}$, at most two times. This follows easily from the properties of $\tau$ and from the observation that at most two interpolated lines of the same frequency may have endpoints interpolating not disjoint sets of space vertices.

We can now complete also the proof of the bound (5.60). It is sufficient to show that, given $\varepsilon>0$,

$$
\begin{aligned}
& \frac{1}{|\Lambda|} \int d \underline{x}_{v_{0}} \prod_{\substack{\mathrm{n} \mathrm{t} v \geq v_{0} \\
v \text { not e } \mathrm{p}}}\left[\frac{1}{s_{v} !} \sum_{\tilde{T}_{v}} \prod_{l \in \tilde{T}_{v}} e^{-\varepsilon \gamma^{h_{l}}\left|x_{l}-y_{l}\right|}\right] \\
& \leq \prod_{v} C^{\sum_{v}^{s_{v}} N_{v^{i}}} \gamma^{-2 h_{v}\left(s_{v}-1\right)},
\end{aligned}
$$

where $\tilde{T}_{v}$ is the anchored tree corresponding to $T_{v}, v^{1}, \ldots, v^{s_{v}}$ are the non-trivial vertices immediately following $v$, and $N_{v^{\imath}}=\left|P_{v^{2}}\right|-\left|Q_{v^{i}}\right|$ is the number of the external lines in $v^{2}$.

If we fix in an arbitrary way a point in $x_{v_{0}}$, we can bound the other integrations in the 1.h.s. of (A3.18) as usual, starting from the endpoints of $\tilde{T}$, and we get

$$
\prod_{\substack{\mathrm{n} t v \geq v_{0} \\ v \text { not e p }}}\left(C \gamma^{-2 h_{v}}\right)^{s_{v}-1} \frac{1}{s_{v} !}\left|\tilde{T}_{v}\right|
$$

where $\left|\tilde{T}_{v}\right|$ is the number of possible choice for $\tilde{T}_{v}$, which can be bounded in the standard way, by observing that the number of anchored trees with $d_{i}$ lines branching 
from the vertex $v^{i}$ can be bounded by:

$$
\frac{\left(s_{v}-2\right) !}{\left(d_{1}-1\right) ! \ldots\left(d_{s_{v}}-1\right) !} N_{v^{1}}^{d_{1}} \cdot N_{v^{s_{v}}}^{d_{s_{v}}} .
$$

The bound (A3.18) easily follows from (A3.19) and (A3.20).

\section{Appendix 4: Simplified Beta Functional}

To show that the ratios $Z_{h} / Z_{h^{\prime}}$ can be eliminated we remark that they can be computed recursively, from (5.47), (5.48), provided (5.51) holds. On the other hand, if we suppose that $\left|r_{h}\right|<\varepsilon$ for all $h>k$ then it follows from the bounds of Sect. 5 and from (5.54) that $\left|Z_{h+1}\right| Z_{h} \mid<e^{c_{2} \varepsilon^{2}}$ for $h>k$. Hence the ratios $Z_{h+1} / Z_{h}$, regarded as recursively defined functions of $r_{h+1}, ., r_{0}$, are holomorphic in the domain $\left|r_{j}\right|<\varepsilon$, $j>h$. It follows that the r.h.s. of (5.48), as a function of $r_{h}, h>k$, is holomorphic in the domain $\left|r_{h}\right|<\varepsilon$.

In this appendix we prove Eqs. (7.2). Let us consider the second of (7.1) for $h=-1$ :

$$
\nu_{-1}=\gamma \nu_{0}+B^{0, \nu}\left(\mu_{0}, \nu_{0}\right)
$$

with $B^{0, \nu}$ holomorphic in $\mu_{0}, \nu_{0}$ for $\left|\mu_{0}\right|,\left|\nu_{0}\right|<\varepsilon$, and

$$
\sup _{\left|\mu_{0}\right|,\left|\nu_{0}\right|<\varepsilon}\left|B^{0, \nu}\left(\mu_{0}, \nu_{0}\right)\right|<b \varepsilon^{2}
$$

for some $b>0$.

The image of the disk $\left|\nu_{0}\right|<\varepsilon$ under the map $\nu_{0} \rightarrow \gamma \nu_{0}+B^{0, \nu}\left(\mu_{0}, \nu_{0}\right)$ will contain the disk of radius $r=\gamma \varepsilon-b \varepsilon^{2}$, which is larger than $\varepsilon$, if $\varepsilon \leq \bar{\varepsilon}=(\gamma-1) / b$, as we shall suppose from now on.

Hence for all $\left|\nu_{-1}\right|<\varepsilon$ there is a point $\nu_{0}$ with $\left|\nu_{0}\right|<\varepsilon$ such that (A4.1) holds: such a point is clearly unique if $\varepsilon$ is small enough. Then (A4.1) can be inverted in the form

$$
\nu_{0}=\gamma^{-1} \nu_{-1}+C\left(\nu_{-1}, \mu_{0}\right)
$$

with $C\left(\nu_{-1}, \mu_{0}\right)$ holomorphic if $\left|\nu_{-1}\right|,\left|\mu_{0}\right|<\varepsilon$ and

$$
\left|C\left(\nu_{-1}, \mu_{0}\right)\right|=\gamma^{-1}\left|B^{0, \nu}\left(\mu_{0}, \nu_{0}\right)\right| \leq b \varepsilon^{2} \gamma^{-1} .
$$

In fact we see that the analyticity domain in $\nu_{-1}$ of $C\left(\nu_{-1}, \mu_{0}\right)$ could be taken as large as $\varepsilon \gamma^{1-\xi}$ with $\xi>0$ prefixed and for $\varepsilon$ small enough (depending on $\xi$ ).

Let us consider now the equation:

$$
\nu_{-2}=\gamma \nu_{-1}+B^{-2, \nu}\left(\mu_{-1}, \nu_{-1}, \mu_{0}, \gamma^{-1} \nu_{-1}+C\left(\nu_{-1}, \mu_{0}\right)\right)
$$

Equation (A4.4) has the same form as (A4.1) if one sets

$$
B\left(\nu_{-1}, \mu_{-1}, \mu_{0}\right)=B^{-2, \nu}\left(\mu_{-1}, \nu_{-1}, \mu_{0}, \gamma^{-1} \nu_{-1}+C\left(\nu_{-1}, \mu_{0}\right)\right)
$$

and $B$ verifies the bound $b \varepsilon^{2}$ and $b$ can be taken to be the same $b$ as in (A4.2), by the bounds of Sect. 5; hence we can proceed inductively. 
By repeating the argument we arrive at

$$
\nu_{h-1}=\gamma \nu_{h}+\mathscr{R}_{\nu}^{h}\left(\mu_{h}, \mu_{h+1}, \ldots, \mu_{0} ; \nu_{h}\right)
$$

with $\mathscr{R}_{\nu}^{h}$ analytic for $\left|\mu_{h^{\prime}}\right|<\varepsilon, h^{\prime} \geq h$, and $\left|\nu_{h}\right|<\varepsilon$. And, by the same substitutions, we get also

$$
\mu_{h-1}=\mu_{h}+\mathscr{B}_{\mu}^{h}\left(\mu_{h}, \mu_{h+1}, ., \mu_{0} ; \nu_{h}\right)
$$

with $\mathscr{B}_{\mu}^{h}$ analytic for $\left|\mu_{h^{\prime}}\right|<\varepsilon, h^{\prime} \geq h$, and $\left|\nu_{h}\right|<\varepsilon$.

Acknowledgements GG is indebted to Giovanni Felder for introducing him to the anomalous dimension in $\varphi^{4}$ field theory We thank G Gentile, V Mastropietro, and W Metzner for many useful discussions. We achknowledge support from Ministero della Ricerca Scientifica, from Gruppo Nazionale della Fisica Matematica, from C.N R (BS), from Rutgers University (GG and BS) and from Institut des Hautes Études Scientifiques, Paris (GG)

\section{References}

[B] Brydges, D.: A short course on cluster expansions Les Houches 1984, K Osterwalder, R Stora (eds.) Amsterdam: North-Holland (1986)

[BG] Benfatto, G, Gallavotti, G : Perturbation Theory of the Fermi surface in a quantum liquid. A general quasiparticle formalism and one dimensional systems. J Stat Phys. 59, 541-664 (1990)

[BGM] Benfatto, G., Gallavotti, G, Mastropietro, V.: Renormalization group and the Fermi surface in the Luttinger model Phys Rev B 45, 5468-5480 (1992)

[dV] Da Veiga, P: Construction de modèles non renormalizables en théorie quantique des champs. Thèse, Universitè d'Orsay (1991)

[DM] Di Castro, C, Metzner, W : Ward identities and the $\beta$ function in the Luttinger liquid Phys Rev Lett 67, 3852-3855 (1991)

[F] Felder, G : Construction of a non-trivial planar field theory with ultraviolet stable fixed point Commun Math. Phys. 102, 139-155 (1985)

[FG] Felder, G., Gallavotti, G : Perturbation theory and non-renormalizable scalar fields Commun Math Phys 102, 549-571 (1985)

[FMRS] Feldman, J, Magnen, J, Rivasseau, V, Sénéor, R.: The massive Gross-Neveu model in two dimensions Commun Math Phys 103, 67-103 (1986)

[FMRT] Feldmann, J, Magnen, J., Rivasseau, V, Trubowitz, E: An infinite volume expansion for many fermion Green's functions. Helv Phys Acta 65, 679-721 (1992)

[FT1] Feldman, J, Trubowitz, E.: Perturbation theory for many fermion systems Helv Phys Acta 63, 156-260 (1990)

[FT2] Feldman, J, Trubowitz, E : The flow of an electron-phonon system to the superconducting state. Helv Phys Acta 64, 213-357 (1991)

[G] Gallavotti, G.: Renormalization theory and ultraviolet stability for scalar fields via renormalization group methods. Rev. Mod Phys 57, 471-562 (1985)

[GK1] Gawedzki, K., Kupiainen, A.: Gross-Neveu model through convergent perturbation expansion Commun Math. Phys 102, 1-30 (1985)

[GK2] Gawedzki, K, Kupiainen, A : Renormalization of a non-renormalizable quantum field theory Nucl Phys B 262, 33-48 (1985)

[GK3] Gawedzki, K, Kupiainen, A.: Massless lattice $\varphi_{4}^{4}$ theory: rigorous control of a renormalizable asymptotically free model Commun Math Phys 99, 197-252 (1985)

[GN] Gallavotti, G, Nicolò, F.: Renormalization theory in four-dimensional scalar fields I, II Commun Math Phys 100, 545-590 and 101, 1-36 (1985)

[GS] Gentile, G, Scoppola, B.: CARR preprint n 17/92; to appear in Commun Math Phys

[L] Luttinger, J : An exact soluble model of a many-fermions system. J Math Phys 4, 1154 $1162(1963)$ 
[Le] Lesniewski, A.: Effective action for the Yukawa 2 quantum field theory Commun. Math Phys 108, 437-467 (1987)

[ML] Mattis, D, Lieb, E : Exact solution of a many fermion system and its associated boson fields J Math. Phys 6, 304-312 (1965)

[NO] Negele, J W., Orland, H : Quantum many-particle systems New York: Addison-Wesley, 1987

[O'] O'Carrol, M., Pereira, E A : Correlation function formulas for some infrared asymptoticfree scalar field lattice models via block renormalization group Lett Math Phys 23, 1-9 (1992)

[R] Ruelle, D : Statistical mechanics: rigorous results New York: Benjamin 1969

[S] Solyom, J : The Fermi gas model of one dimensional conductors. Adv Phys 28, 201-303 (1979)

[T] Tomonaga, S : Remarks on Bloch's methods of sound waves applied to many fermion problems. Progr. Theor Phys. 5, 554-569 (1950)

[Th] Thirring, W : A Course in Mathematical Physics, Vol 4 New York: Springer 1983

[WF] Wilson, K, Fisher, M : Critical exponents in 3.99 dimensions Phys Rev. Lett 28, 240-243 (1970)

Communicated by G. Felder 
